Wolfgang Laux

Erhöhung

staatswirtschaftlicher

Effizienz durch

budgetäre

Selbstbeschränkung? 


\section{Erhöhung staatswirtschaftlicher Effizienz durch budgetäre Selbstbeschränkung?}

Die Arbeit nimmt die in jüngster Zeit vor allem in den USA erhobenen Forderungen nach einer verfassungsmäßigen Begrenzung der Staatstätigkeit zum Ausgangspunkt für eine systematische Analyse der Vor- und Nachteile einer institutionellen Fixierung der Staatsquote. Grundlagen sind dabei zum einen eine Theorie des Staatsversagens, zum anderen ein politisches Modell, in dem das Angebot an öffentlichen Gütern aus der Beziehung zwischen Politikern und Bürokratien und die Nachfrage aus der Beziehung zwischen Bürgern und Politikern erklärt wird. Im Ergebnis erscheint eine Begrenzungsregel günstig für die Qualität des Angebots aber unsicher in Bezug auf die Qualität der Nachfrage nach öffentlichen Gütern.

Wolfgang Laux wurde 1954 in Andernach geboren. 1974-1979 Studium der Volkswirtschaftslehre an der Freien Universität Berlin; seit 1979 wissenschaftlicher Mitarbeiter am Institut für Finanzen, Steuern und Sozialpolitik der Freien Universität Berlin. 
Erhöhung staatswirtschaftlicher Effizienz durch budgetäre Selbstbeschränkung? 


\section{FINANZWISSENSCHAFTLICHE SCHRIFTEN}

Herausgegeben von den Professoren

Albers, Krause-Junk, Littmann, Oberhauser, Pohmer, Schmıdt

Band 24

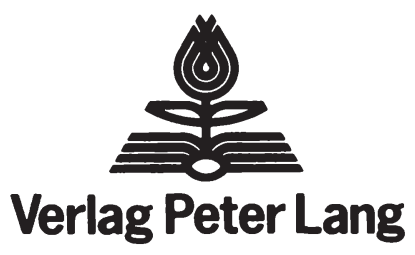

Frankfurt am Maın - Bern - New York - Nancy 


\section{Wolfgang Laux}

\section{Erhöhung \\ staatswirtschaftlicherEffizienz \\ durch budgetäre \\ Selbstbeschränkung? \\ Zur Idee einer verfassungsmäßig \\ verankerten Ausgabengrenze}

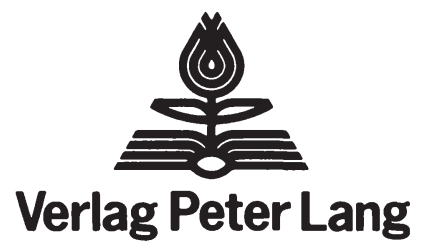

Frankfurt am Maın - Bern - New York - Nancy 
CIP-Kurztitelaufnahme der Deutschen Bıbliothek

\section{Laux, Wolfgang:}

Erhöhung staatswirtschaftlicher Effizıenz durch budgetäre Selbstbeschränkung? : Zur Idee e. verfassungsmässig verankerten Ausgabengrenze / Wolfgang Laux. - Frankfurt am Main ; Bern ; New York, Nancy : Lang, 1984.

(Finanzwissenschaftliche Schriften ; Bd. 24)

ISBN 3-8204-5347-4

NE: GT

Open Access: The online version of this publication is published on www.peterlang.com and www.econstor.eu under the international Creative Commons License CC-BY 4.0. Learn more on how you can use and share this work: http://creativecommons.org/licenses/by/4.0.

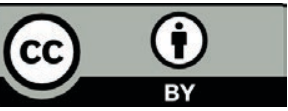

This book is available Open Access thanks to the kind support of ZBW - Leibniz-Informationszentrum Wirtschaft.

D 188

ISSN 0170-8252

ISBN 3-8204-5347-4

ISBN 978-3-631-75220-3 (eBook)

(C) Verlag Peter Lang GmbH, Frankfurt am Main 1984

Alle Rechte vorbehalten.

Nachdruck oder Vervielfältigung, auch auszugsweise, in allen Formen wie Mikrofilm, Xerographie, Mikrofiche, Mikrocard, Offset verboten.

Druck und Bindung: Weihert-Druck GmbH, Darmstadt 
Inhalt

1. Einführung: Steuerzahlerrevolte und die Forderung nach Budgetbegrenzungen

1.1. Zur Geschichte und Motivation der Steuerzahlerrevolte in den USA 2

1.2 Das Modell des Leviathan-States 9

2. Theorie des Statsversagens und die Rationalität budgetärer Beschränkungen 16 2.1. Zum Begriff "Staatsversagen" 16

2.1.1. Zur Problematik des prozessualen Staatsversagens $\quad 17$

2.1.2. Zur Problematik des situationsbezogenen Statsversagens 22 2.1.2.1 Der Begriff der Belastung durch den Stat 22 2.1.2.2 Eine Definition situationsbezogenen Staatsversagens

2.2. Grunduberlegungen zur Rationalität von Budgetgrenzen als konstitutionelle Regelungen

2.3. Die verschiedenen Formen der Budgetbeschränkungen

2.3.1. Verfahrensmäßige Beschränkungen 46

2.3.2. Ergebnisbezogene oder quantitative Budgetbegrenzungen

3. Politische Entscheidungsmechanismen und Budgetgrenzen

3.1. Das Grundmodell des staatlichen Bereitstellungsprozesses 
3.2. Das Angebot an statlichen Leistungen 64 3.2.1. Das Angebotsuerhalten von Bürokratien

64

3.2.1.1 Das Grundmodell Niskanens

3.2.1.2 Bürokratie und diskretionäres Budget $\quad 73$

3.2.1.3 Ein gemischtes Zielsystem

3.2.1.4 Bürokratie und politische Kontrolle

3.2.2. Budgetbeschränkungen und Bürokratie 79

3.2.2.1 Der NISKANEN-Ansatz 79

3.2.2.2 Die Budgetbeschränkung im MIGUE-BELANGER-Fal1 84

3.2.2.3 Budgetbeschränkung und gemischtes Zielsystem 88

3.2.2.4 Budgetgrenze und Kontrollkosten

3.2.3. Fazit

3.3. Die Nachfrage nach Staatsleistungen im "Public-Choice"-Paradigma

3.3.1. Vollkommene politische Konkurrenz

3.3.2. Die Bedeutung der Information

3.3.3 InteressengruppeneinfluB und unvollständige Information

3.3.4. Politischer EinfluB und Staatsversagen

3.3.4.1 Politischer Einfluß und prozessuales Staatsversagen

3.3.4.2 Ein Gleichgewichtsmodell des politischen Einflusses 
3.3.4.3 Situationsbezogenes

Staatsversagen und politischer EinfluB

3.3.4.4 Die Behandlung der

Fiskalillusion in

der Literatur

3.3.5. Exkurs: Burrokratie als

Interessengruppe

3.3.5.1 Zum Abstimmungsverhalten der Bürokratie 139

3.3.5.2 Die Programmformulierung

3.3.5.3 Bürokratie und Information

3.3.6 Die Auswirkung von Budgetbeschränkungen auf den politischen ProzeB

3.3.6.1 Die Ressourcen zur Transfererlangung

3.3.6.2 Reduktion oder Erhöhung situationsbezogener Ineffizienz?

3.3.6.3 Die Problematik von Auswe ichmöglichkeiten

4. SchluB 
Wolfgang Laux - 978-3-631-75220-3

Downloaded from PubFactory at 01/11/2019 06:56:13AM

via free access 


\section{Einführung: Steuerzahlerrevolte und die Forderung nach Budgetbegrenzung}

Ausgangspunkt und $A n l a B$ dieser Arbeit ist das Phänomen der sogenannten "Steuerzahlerrevolte" in den USA der späten 70 er Jahre, deren Höhepunkt wohl die Verabschiedung der "Proposition 13" in Californien im Jahr 1978 war. Auch wenn die Bewegung anscheinend im Augenblick an Aktualität und Durchschlagskraft verloren hat, den durch sie induzierten Maßnahmen teilweise auch Erfolglosigkeit bescheinigt wurde, so war und ist doch die Resonanz im politischen und auch im wissenschaftlichen Bereich enorm. Insbesondere gaben die Ergebnisse der Steuerzahlerrevolte AnlaB zu grundsätzlichen Oberlegungen über die Rolle des Staates in der Wirtschaft.

Der Ansatzpunkt unterscheidet sich dabei von dem normalerweise im Zusammenhang mit einer Diskussion um den Sinn der Staatstätigkeit gewählten. Hier ging man von bestimmten Vorstellungen uber die ziele staatlichen Handelns aus und diskutierte das Für und Wider einzelner MaBnahmen des Staates. Dabei gingen Befürworter wie Kritiker von denselben impliziten Voraussetzungen aus: Beide Seiten unterstellten, daB der Staat auf bestimmte Unvollkommenheiten des privaten Sektors reagieren sollte und als zielvorstellung einen irgendwie gearteten gesellschaftlichen 0ptimalzustand hatte. Kontrovers waren die Auffassungen über den gesellschaftlichen Optimalzustand, die Höhe des staatlichen Handlungsbedarfs und die Möglichkeiten und Wirkungen, die mit staatlichen Eingriffen verbunden waren. Immer aber lag der Diskussion die eine oder andere Ansicht darüber zugrunde, was der Stat tun sollte. 
Die neuere Kritik, die im Zusammenhang mit der Steuerzahlerrevolte artikuliert wurde, beschreitet einen anderen Weg: Die Bestimmungsgründe und die Entstehungsgeschichte der bestehenden staatlichen Ziele werden in den Mittelpunkt der Untersuchung gestellt. Die Kritik geht somit einen Schritt weiter: Es werden nicht nur bestimmte Normen fur statiches Handeln postuliert, sondern die Genese der bestehenden Normen erklärt und daraus Konsequenzen für eine Umgestaltung des politischen Prozesses gezogen.

1.1 Zur Geschichte und Motivation der Steuerzahlerrevolte in den USA

Steuerprotestbewegungen sind an sich nichts Neues und auch nichts Ungewöhnliches. Gerade in den USA haben Steuerrevolten eine lange Tradition.1) Einen Höhepunkt fanden Bewegungen zur Begrenzung bzw. Reduzierung der Steuer lasten in den 70er Jahren in den USA wie in Europa, wobe in den USA das Jahr 1978 mit dem Erfolg der Proposition 13 in Californien wohl den Höhepunkt darstellte.

Proposition 13 ist ein Bürgerbegehren auf eine Ergänzung zur Verfassung von Californien. Inhalt dieser Ergänzung ist eine Begrenzung des Steuersatzes der property tax ${ }^{2}$ ) auf $1 \%$ des Marktwertes sowie Bestimmungen, die eine

1) Auch die"Boston Tea Party" als AnlaB des amerikanischen Unabhängigkeitskrieges stellt vordergrüdig eine Steuerrevolte dar. Zur Geschichte des Steuerprotestes in den USA vgl. REID (1979)

2) Die property tax ist eine Steuer auf Grundvermögen und macht im Durchschnitt $80 \%$ der Steuereinnahmen der Gemeinden aus. Der vorher bestehende durchschnittiche Satz lag bei $2,5 \%$. 
2/3-Mehrheit in den gesetzgebenden Körperschaften Californiens für Erhöhungen von Steuern zur Voraussetzung machen.

Proposition 13 war nicht die erste Initiative, die sich gegen die property tax wandte.1) In den Jahren 1968 und 1972 waren Gesetze erlassen worden, die eine Reduktion der Steuerbelastung durch Freibeträge und eine Begrenzung der Steuersätze auf $2,5 \%$ beinhalteten. Das Gesetz von 1972 war dabei eine Gegenmaßnahme auf die (gescheiterte) WatsonInitiative, die unter anderem eine Begrenzung des Steuersatzes auf $1,75 \%$ anstrebte. 1974 unterlag die von Gouverneur Reagan unterstütze Proposition 1, nach der die bestehenden property-tax-Sätze zu Maximal-Sätzen erklärt wurden, und die eine Begrenzung des Wachstums der Ausgaben des States Californien auf die Wachstumsrate des persönlichen Einkommens beinhaltete, in einer Abstimmung nur knapp.

Der Erfolg von Proposition 13 in Californien hatte eine Reihe weiterer Initiativen bzw. Gesetzesveränderungen in anderen US-Staaten zur Folge.2) Im November 1978 kamen in 8 Staaten ähnliche Initiativen zur Abstimmung, die nur in 2 Staten (Maryland, Oregon) scheiterten.

Im einzelnen führten diese Abstimmungen nicht immer zu direkten Verfassungsänderungen, da diese Möglichkeit nicht in allen US-amerikanischen Staaten besteht, sondern zu "normalen" gesetzlichen Regelungen. Auch lag die Initiative zu einer Verfassungs - bzw. Gesetzesänderung nicht immer bei einer (außerparlamentarischen) Burgerbewegung, sondern teilweise auch bei den gesetzgebenden Körperschaften selbst (so in Alabama). Unabhängig davon waren
I) Zum folgenden siehe KIRLIN (1982) S. $45 \mathrm{ff}$.
2) DazU DWORAK (1980) S. $87 \mathrm{ff}$. 
die verschiedenen zur Abstimmung gestellten Vorschläge vom Inhalt her sehr ähnlich: Die wesentlichen Punkte waren eine Beschneidung des property-tax-Satzes,2/3-Mehrheiten für steuererhöhende Gesetzesänderungen in den gesetzgebenden Körperschaften, sehr oft eine Begrenzung der Wachstumsrate des Steueraufkommens.

Insgesamt erreichte die Steuerzahlerrevolte, daB die Mehrzahl der amerikanischen Bundesstaaten die eine oder andere Art einer fiskalischen Begrenzung einführte.1) Auf nationaler Ebene fand die Steuerzahlerrevolte in zwei Aktivitäten ihren Ausdruck: Die eine richtete sich auf die Einfuihrung einer allgemeinen Ausgabengrenze für den Bundeshaushalt. Diese Begrenzung der Staatsausgaben sollte als Zusatz in die Verfassung aufgenommen werden und hatte die Unterstiutzung verschiedener Steuerzahlerorganisationen, fand aber keine hinreichende legislative Unterstützung. Ein dabei vom National Tax Limitation Committee vorgeschlagener Verfassungszusatz hatte folgenden Inhalt:2) Die Zuwachsrate der Staatsausgaben wird auf die Zuwachsrate des nominellen Sozialprodukts des Vorjahres begrenzt, 3 ) wobei unter Ausgaben auch die "off-budget outlays", also Ausgaben auBerhalb des eigentlichen Haushalts, nicht jedoch die Rückzahlung von Schulden subsumiert wurden. Eine Anderung der Ausgabengrenze ist nur möglich mit einer 3/4-Mehrheit beider Häuser des Kongresses, also sowohl des Abgeordnetenhauses als auch

i) siehe FOLKERS (1983a) S. 16; zum Stand Ende 1979 siehe KIRLIN (1982) S. $33 / 34$

2) siehe WILDAVSKY (1980) S. 127

3) Tatsächlich handelt es sich nicht um eine nahtlose Anknüpfung. Die Ausgaben eines Fiskaljahres werden an das Wachstum des Kalenderjahres gekoppelt, das dem Beginn dieses Fiskaljahres vorausging. Das jeweilige Fiskaljahr beginnt aber im letzten Drittel eines Kalenderjahres, woraus sich eine Verzögerung von einem dreiviertel Jahr ergibt. 
des Senats. Im Fall einer Erklärung des nationalen Notstands durch den Präsidenten kann mit einer 2/3-Mehrheit beider Häuser eine Oberschreitung der Ausgabengrenze für ein Jahr beschlossen werden.

Eine besondere Rolle spielt bei diesem Vorschlag die Behandlung rein nomineller Sozialproduktzuwächse. So sollen bei einem Steigen des Preisniveaus über drei Prozent für jeden darüber liegenden Prozentpunkt die Ausgaben nur um dreiviertel Prozent steigen durfen. Desweiteren findet man Bestimmungen, die verhindern sollen, $d a B$ eine Ausdehnung der Ausgaben des Zentralstates zu Lasten der Zuweisungen an die Einzelstaten stattfindet bzw. der Zentralstaat eigene Aufgaben durch die Einzelstaaten finanzieren läßt.

Die Anwendung dieser Regelung würde die derzeit bestehende Staatsausgabenquote als obergrenze festschreiben. Da die Limitierung auf die Zuwächse bezogen ist, führt eine einmalige Unterschreitung des maximalen Ausgabenspielraums $z u$ einem bleibenden Effekt: Die reduzierte Ausgabenquote wird bindend auch für alle folgenden Perioden. Die Besonderheit der Behandlung der Inflation in diesem Ansatz ergibt sich daraus, daB dem Staat ein positiver Anreiz zur Inflationsbekämpfung gegeben werden sollte.

Das National Tax Limitation Committee versuchte auf verschiedene Weise, seine Vorstellungen dem Kongress gegenüber zur Geltung zu bringen (Postkartenaktionen an Abgeordnete, eine Petition an den Präsidenten), 1) ohne daB allerdings ein sich in einer Verfassungsänderung niederschlagender Erfolg verbucht werden konnte.

i) Vgl. DWORAK (1980) S. $129 \mathrm{ff}$. 
Einen größeren politischen Erfolg erzielte eine vom Senat ausgehende Initiative. Nach der amerikanischen Verfassung muB eine verfassungsändernde Versammlung einberufen werden, wenn $2 / 3$ der Bundesstaaten (also 34 ) dies fordern. Im Januar 1982 unterstützten 31 Staten einen solchen Schritt, wobei der zur Diskussion gestellte Punkt der Verfassungsänderung im wesentlichen die Forderung nach einem materiellen Haushaltsausgleich war. Die im "senate joint resolution 58" geforderte Anderung beinhaltete im einzelnen einen jährlichen Ausgleich der (geplanten) Ausgaben und Einnahmen, wobei der Einnahmebegriff nicht die Schuldenaufnahme einschloB und Schuldenrückzahlungen nicht als Ausgaben angesehen wurden. Zudem galt als obergrenze für das Wachstum der Einnahmen, soweit dies nicht auf die Verabschiedung eines speziellen Gesetzes zurückzuführen ist, das Wachstum des Sozialprodukts des Vorjahres. Ausnahmen waren für Kriegszeiten zugelassen.

Letztendlich fanden sich doch nicht genügend Staten bereit, die Einberufung einer verfassungsgebenden Versammlung zu unterstützen, so daB auch diesem Vorschlag kein Erfolg beschieden war. 1 )

Bemerkenswert an der Steuerzahlerrevolte ist vor allen Dingen, daB, soweit möglich, die Form einer konstitutionellen Veränderung zur Einschränkung von Staatsausgaben oder Steuern gewählt wurde. Offensichtlich wurde dem

1) Möglicherweise war dies auch gar nicht gewünscht, sondern die Staten versuchten durch diese Maßnahme Druck auf das Abgeordnetenhaus auszuüben, selbst etwas zu unternehmen. Die Einberufung einer verfassungs gebenden Versammlung wäre auch ohne Beispiel in der amerikanischen Geschichte gewesen und hätte sich zudem nicht auf den angesprochenen Punkt beschränken lassen, da de jure eine einmal einberufene verfassungsgebende Versammlung sich mit jedem beliebigen Problem beschäftigen konnte. Vergleiche dazu GUNTHER (1980). 
"normalen" politischen ProzeB und den darin dominierenden Parteien ein effektives Handeln auf eine Begrenzung der Statstätigkeit hin nicht zugetraut.

In Europa artikulierte sich der Steuerprotest, teilweise sogar bevor die amerikanische Entwicklung soviel Aufmerksamkeit auf sich zog, in der Bildung eigener Parteien, deren einziges oder zumindest Hauptziel eine Verringerung der Steuerlast war und die in Nordeuropa teilweise erstaunliche Wahlerfolge erzielen konnten (besonders die Fortschrittspartej des Steueranwalts Glistrup in Dänemark).1)

Allerdings ist nicht eindeutig, ob die Steuerprotestbewegungen als grundsätzliche Kritik an der Statstätigkeit bzw. dem Umfang staatlicher Leistungen angesehen werden können. Gerade in Californien durfte weniger eine Kritik an der Stadstätigkeit überhaupt, sondern an der speziellen Form der Einnahmeerhebung über die property tax im Mittelpunkt des Protests der Steuerzahler gelegen haben. Die property tax erfuhr besonders in Californien durch die direkte Koppelung an die stark gestiegenen Immobilienpreise eine recht hohe Steigerung, und da es sich um eine einkommensunabhängige Steuer handelt, kamen bei sinkendem Einkommen und wirtschaftlicher Stagnation viele Hauseigentuimer in Zahlungsschwierigkeiten bzw. wurde die propertytax-Belastung als besonders drückend empfunden.

Empirische Untersuchungen 2 ) haben festgestellt, daB eine generelle Unzufriedenheit mit der Staatstätigkeit nur auf dem Sektor der (umverteilend wirkenden) Wohlfahrtsausgaben

i) Vgl. MURPHY/RUBART/MOLLER/RASCHKE (1979)

2) Fuir Californien: CITRIN (1979), SHAPIRO (1981), SHAPIRO/PURYEAR/ROSS (1979); für Michigan: COURANT / GRAML I CH/RUB INFELD (1979), (1981), FREIMAN/GRASSO (1982); Obersicht bei FOLKERS (1983a), Kapitel 3 
bestand. Bei den meisten anderen Statsaktivitäten fand sich zwar immer eine Minderheit für eine Einschränkung, die Mehrheit wiunschte aber keine Reduzierung der staatlichen Leistungen. Allerdings wurde auch der generelle Verdacht geäuBert, der staat produziere ineffizient, und damit gekoppelt die Vermutung, eine Reduktion der Steuerlasten sei auch bei gleichbleibenden statichen Leistungen möglich, wenn nur die Ineffizienzen innerhalb des öffentlichen Sektors entsprechend vermindert wirden.

Wenn somit die Motive des Steuerzahlerprotests wohl eher darin lagen, daß bestimmte Steuerzahlergruppen auf Kosten anderer (Steuerzahler oder Zahlungsempfänger) Einkommensvorteile realisieren wollten, so bleibt doch der Weg, der dabei gewählt wurde, bemerkenswert. Offensicht 1 ich wurde keine der existierenden Parteien durch Wählervotum beauftragt, diese Lastverteilungsänderungen vorzunehmen, sondern der Weg der direkten Einflußnahme eingeschlagen. Die existierenden Parteien wurden bereits als zu sehr in die Statstätigkeit integriert angesehen, um ein solches radikales Ansinnen zu vertreten.

\subsection{Das Model1 des Leviathan-Staates}

Die Möglichkeiten bzw. Bedingungen einer Steuerzahlerrevolte waren bereits von der wissenschaft antizipiert worden.1) Auch die Möglichkeit der Einführung verfassungsmäßiger Budget-, speziell Aufkommensgrenzen war bereits in Ansätzen entwickelt, bevor es zu der Entwicklung in Californien kam. Bereits 1977 veröffentichten BRENNAN und BUCHANAN einen Aufsatz: "Zu einer Steuerver-

1) vgl. BUCHANAN/FLOWERS (1969), JACKSON (1972) 
fassung für den Leviathan", in dem ein Grundmodell der Statseingriffe und die Möglichkeiten der Begrenzung der Statstätigkeit durch Verfassungsregeln entworfen wurde. Weiter ausgeführt wurden die Gedankengänge dieser Autoren in dem 1980 erschienenen Buch "The Power to Tax. Analytical Foundations of a Fiscal Constitution". Dieses Buch ist nicht der einzige Beitrag zum Komplex der fiskalischen Begrenzungen der Statstätigkeit, vermutlich aber der radikalste und soll deshalb hier vorgestellt werden.

BRENNAN/BUCHANAN's Bestimmung der Statsfunktion leitet sich ab aus dem, was sie den "economic approach" nennen: Der Stat ist eine Institution mit einer bestimmten Ausgestaltung, in der Personen und Gruppen agieren und ihre eigenen Interessen verfolgen, und der auch nur zu diesem Zweck errichtet wurde bzw. daraus legitimiert werden kann. Ein Staat, der als unabhängige Instanz über die gesellschaftliche Wohlfahrt wacht, stellt nach BRENNAN/BUCHANAN eine unzutreffende Fiktion dar.

Ihrer Meinung nach ist das Bild des "Leviathan-Staates" eine zutreffendere Beschreibung des Ist-Zustandes. Der Stat tritt als von einer Politiker-Bürokratenclique beherrschtes monopolistisches Gebilde auf, dessen Macht durch Wahlprozesse und politische Kontrolle nur minimal eingeschränkt ist und dessen $Z$ iel darin besteht, möglichst viele Ressourcen aus den Taschen der Staatsbürger in die eigenen umzuleiten. Der Staat wird als Einnahmemaximierer beschrieben, dem die staatsbürger mehr oder weniger hilflos ausgesetzt sind und dessen Machtfuille durch eine konstitutionelle Regelung zu begrenzen ist. 
Die Zielfunktion des Leviathans läßt sich relativ einfach beschreiben: Maximierung des dem Staat zur Verfügung stehenden (Steuer-)Aufkommens ( $S$ ), definiert als Differenz zwischen Gesamteinnahmen $(R)$ und den zur Erstellung öffentlicher Leistungen notwendigen Ausgaben $(G)$ :

$S=R-G$

Dabei können die Staatsausgaben für öffentliche Leistungen vereinfacht als konstanter Anteil a an den Einnahmen angesehen werden. Unter dieser Voraussetzung diskutieren BRENNAN/BUCHANAN dann, wie das finanzpolitische Instrumentarium aussehen könnte, das man dem Staat (durch eine verfassungsmäßige Einigung) in die Hand geben dürte, damit er seine $Z$ iele möglichst nicht verwirklichen kann, d.h. um den Schaden, den er anrichtet, möglichst klein zu halten.

Da das $Z$ iel des Leviathans die Einnahmenmaximierung ist, wird für BRENNAN/BUCHANAN zum Kriterium der finanzwirtschaftlichen Institutionen, ob diese eine hohe oder eine geringe Möglichkeit zur Ausbeutung der Staatsbürger mit sich bringen. Das Buch liest sich teilweise wie ein Antilehrbuch der Finanzwissenschaft.1) So werden "broadbased-taxes" abgelehnt und die Beschränkung des States auf Einnahmequellen gefordert, die möglichst unergiebig sind bzw. bei denen eine steuerausweichung einfach ist. Die Steuerbemessungsgrundlagen sollten möglichst eng definiert sein und sich auf zum Konsum öffentlicher Güter komplementäre Aktivitäten erstrecken. BRENNAN/BUCHANAN versprechen sich damit eine Erhöhung des Anteils der Steuereinnahmen, die als öffentliche Leistungen wieder an die Bürger zurückfließen. Dadurch, daB durch eine verstärkte Bereitstellung öffentlicher Güter auch die

i) Für einen Vergleich der traditionellen Besteuerungsprinzipien und denen BRENNAN/BUCHANAN's Vgl. GROSSEKETTLER (1980) 
Steuerbemessungsgrundlage steigt, besteht für den Leviathan ein Anreiz, wenigstens einen Teil der Steuereinnahmen zur Produktion öffentlicher Gïter zu verwenden.

Steuersatzdifferenzierungen nach Güterart und Individuen, wie sie z.B. aus der Theorie der optimalen Besteuerung folgen, werden abgelehnt, da durch eine solche Differenzierung dem Leviathan eine maximale Ausbeutung der Staatsbürger möglich gemacht würde. Aus dem gleichen Grund befürworten BRENNAN/BUCHANAN eine progressive Ausgestaltung der Steuertarife. Ihr Argument lautet, daB das maximale Steueraufkommen durch die Ausweichreaktionen der Steuerzahler begrenzt wird. Die relevante GröBe, auf die die Bürger dabei reagieren, stellt dabei die Höhe des Grenzsteuersatzes dar. Bei regressiven Tarifen ist ceteris paribus derselbe Grenzsteuersatz mit einem höheren durchschnittichen Steuersatz und damit einem größerem Steueraufkommen verbunden als bei progressiver Tarifgestaltung. Besonders scharf wenden sich BRENNAN/BUCHANAN gegen die Möglichkeit des Staates, sich zu verschulden: Dadurch sei der Leviathan in die Lage versetzt, auch mögliche Steuereinnahmen späterer $Z$ eiten, zu denen der Staat als Leviathan nicht mehr existieren mag, bereits heute für sich zu erschließen.

Des weiteren wird ein möglichst hoher Grad an fiskalischer Dezentralisation befürwortet.Dadurch entstehen mehrere Gebietskörperschaften, so daß der Bürger die Möglichkeit hat, durch Standortwechsel dem jeweils schlimmsten "lokalen Leviathan" auszuweichen. Den Ausbeutungsmöglichkeiten der einzelnen Gebietskörperschaften sind damit auch zusammengenommen engere Grenzen gesetzt als einem einzigen zentralen Leviathan. Oberregionale Finanzzuweisungen hingegen werden abgelehnt, da dadurch eine interregionale Zusammenarbeit erzwingbar wird und somit die einzelnen regionalen Körperschaften zu einem gleichlaufenden 
Verhalten veranlaßt werden können, wodurch der einschränkende Effekt, der durch einen (möglichen) Wechsel des Wohnortes auf die Handlungsmöglichkeiten des jeweiligen lokalen Leviathans ausgeht, verloren geht.

Als Therapie schlagen BRENNAN/BUCHANAN eine weite Palette von möglichen Verfassungsregeln vor, die alle mehr oder weniger auf eine Begrenzung der Einnahmemöglichkeiten des Staates hinauslaufen.1) Solche Regelungen auf der Verfassungsebene, die die Handlungsmöglichkeiten des States einschränken, bezeichnen sie als "authentische Steuerreform". Allerdings distanzieren sie sich von dem Gedanken, fiskalische Grenzen als einzige Möglichkeit der Einschränkung Leviathans zu sehen.

Die Oberlegungen BRENNAN/BUCHANAN's, die das analytische Handwerkszeug der traditionellen Finanzwissenschaft bestens beherrschen und souverän zu handhaben wissen, sind provozierend und anregend. Allerdings ist ihre konstruk tion des Leviathan-States eine wohl zu vereinfachende Darstellung der Realität. Es fällt auf, daB dem Bild des Leviathan-Staates auch keine exakte Analyse der politischen Entscheidungsmechanismen zugrunde liegt, es vielmehr ohne besondere Begründung entworfen wird. BRENNAN/BUCHANAN weisen zwar auf einige Unzulänglichkeiten des demokratischen Prozesses hin, die einer Kontrolle des Staates auf "post-konstitutioneller" Ebene im Wege stehen. Letztendich wird jedoch die spezifische Ausgestaltung des Leviathan-States, der von einer Bürokraten-PolitikerClique beherrscht wird und dessen $Z$ iel Maximierung der Einnahmen ist, mehr vorausgesetzt als begründet. Eine solche Verfahrensweise, die die Fiktion des "wohlwollenden" Diktators nur durch die des "übelwollenden" Leviathans ersetzt, befriedigt natürlich nicht.

i) vgl. dazu im folgenden Abschnitt 2.3. 
Allerdings behaupten BRENNAN/BUCHANAN auch nicht, daB das bestehende Staatswesen tatsächlich ein Leviathan sei: Das Leviathan-Bild dient eher als Extrem, an dem dargestellt werden soll, welche Konsequenzen aus einer unlimitierten staatlichen Machtfiulle auftreten könnten und welche Folgen für eine Begrenzung der Handlungsmöglichkeiten des Staates zu ziehen sind. Dennoch: BRENNAN/BUCHANAN sind der Ansicht, daB das derzeitige staatliche Handeln eher so beschrieben werden $k a n n$, als ob der Staat ein einnahmemaximierender Leviathan sei, als durch die Parabel des wohlmeinenden Diktators.

Dafür, daB sie auf einer nur "als-ob"-Fiktion des Staates aufbauen, sind die Folgerungen, die BRENNAN/BUCHANAN ziehen, allerdings etwas zu stark - die Konsequenzen ihrer Analyse sind relativ wenig robust gegenuber nur leichten Variationen in der Annahme der Einnahmemaximierung. Die Folge aus der Einnahmemaximierung ist nämlich, daß die Höhe der Statsquote negativ korreliert ist mit der Wohlfahrt der Bürger, d.h. die vom Stat vereinnahmten Ressourcen sind zum gröBten Teil für die Steuerzahler verloren. Entsprechend ist jede Statsquote gröBer als Null mit einem Wohlfahrtsverlust verbunden und jede Maßnahme, die die Einnahmemöglichkeiten des States verringert, positiv zu bewerten.

Man fragt sich, warum die Bürger sich nicht in anarchistischer Manier auf eine Abschaffung des Staates überhaupt einigen und sich stattdessen mit (halbherzigen) Steuerbegrenzungsmaßnahmen begnügen sollen. Die Begründung, die BRENNAN/BUCHANAN geben, ist etwas schwach. DaB die Mitglieder der Gesellschaft ohne Existenz eines Staates noch schlechter dastehen würden, widerspricht dem Tenor des Buches: Da BRENNAN/BUCHANAN sich nicht mit der Frage einer optimalen Staatsquote auseinandersetzen, bzw. innen 
jede Beschränkung der Staatsquote recht ist, wird eine Reduktion der Steuereinnahmen auf Null ebenfalls von ihrer Analyse gedeckt. Ein Stat ohne Einkommen düfte sich dabei nicht wesentlich von einem nichtexistierenden Stat unterscheiden.

In dieser Arbeit soll das Bild des Leviathans mit etwas mehr Inhalt gefüllt werden. Es geht um eine präzisere Ableitung der Bestimmungsgrïnde des staatlichen Einnahmeund Ausgabeverhaltens aus einer Theorie der politischen Entscheidung, wobei auf den vorhandenen, teilweise bis ins Detail ausgearbeiteten Ansätzen der "ökonomischen Theorie der Politik"aufgebaut wird.

Dabei soll folgendermaßen vorgegangen werden: Im zweiten Kapitel soll im ersten Abschnitt ein Kriterium entwickelt werden, nach dem beurteilt werden kann, inwieweit durch staatliches Handeln ein gesellschaftlicher Optimalzustand hergestellt wird bzw. inwieweit es zu einem "Staatsversagen" kommt. Die Entwicklung eines solchen Kriteriums ist deshalb wichtig, da in dieser Arbeit geprift werden soll, ob der politische EntscheidungsprozeB nach Einführung einer Budgetgrenze "bessere" Ergebnisse hervorbringt als zuvor. Dazu bedarf es aber eines Beurteilungsmaßstabes.

In einem zweiten Abschnitt werden Oberlegungen angestellt, welche grundsätzliche Funktion die von der Steuerprotestbewegung oft geforderte Einführung einer in der Verfassung verankerten Begrenzung der Statstätigkeit erfüllen muß, um ein mögliches "Staatsversagen" zu verhindern oder dessen $A u s m a B$ zu verringern. Es geht dabei um die besonderen Charakteristika, die eine verfassungsmäßige von einer "normalen" Regelung abheben. 
Dabei wird auf die von BUCHANAN/TULLOCK entwickelte Unterscheidung zwischen "konstitutionellen" und "operationalen" Entscheidungen zurückgegriffen: "Konstitutionelle" Entscheidungen, die sich in Verfassungsvorschriften niederschlagen, bestimmen die formalen Ansprüche, die an den Ablauf des Entscheidungsprozesses auf operationaler Ebene, also über bestimmte Sachprobleme, gestellt werden. Es soll gezeigt werden, wie die Forderung nach einer verfassungsmäBig verankerten Budgetgrenze in dieses System eingeordnet werden kann. In einem dritten Abschnitt wird dann ein kurzer $A b r i B$ der verschiedenen Möglichkeiten von Budgetbegrenzungen gegeben.

Im dritten Kapitel werden die Eigenschaften des politischen Entscheidungsprozesses in einer Demokratie näher beschrieben. Es geht darum, die Bestimmungsgründe für ein mögliches Staatsversagen darzulegen und zu untersuchen, ob die Einführung einer verfassungsmäßigen Budgetgrenze dem entgegenwirken kann.

Dabei wird zwischen zwei Ansatzpunkten des Staatsversagens unterschieden. Einmal steht auf der "Angebotsseite" das Verhalten staticher Bürokratien und die daraus resultierenden Wirkungen einer Budgetbegrenzung im Mittelpunkt (Abschnitt 3.2.). Im darauf folgenden Abschnitt wird die "Nachfrageseite" betrachtet: Hier geht es speziell um die Art des Informationsaustausches $z$ wischen Politikern und den Bürgern als Steuerzahlern und Adressaten staatlicher Leistungen. Besonderer Augenmerk gilt dabei dem ProzeB der Einflußnahme von Interessengruppen auf statliche Entscheidungen und den daraus folgenden Ineffizienzen. Darauf aufbauend wird untersucht, ob und inwieweit durch Budgetbegrenzungen eine Reduktion dieser Ineffizienzen möglich ist. 
2. Theorie des Staatsversagens und die Rationalitat budgetärer Beschränkungen

\subsection{Zum Begriff "Staatsversagen"}

Die Entwicklung des Begriffs "Staatsversagen"l) ist vor dem Hintergrund einer Tradition zu sehen, die die Notwendigkeit staatlicher Eingriffe auf den Nachweis bestimmter unerwünschter Ergebnisse des Marktprozesses zurückführte. Dabei wurden die Bedingungen, unter denen es zu einem "Marktversagen" kommt, in den Mittelpunkt der Analyse gestellt, die Voraussetzungen dafür, daß durch ein staatliches Eingreifen ein besseres Ergebnis zustande kommt, aber weithin vernachlässigt.2)

Der Umstand, daß der Nachweis eines nichtoptimalen Funktionierens des Marktprozesses allein nicht ausreicht, um daraus auf eine oberlegenheit staatlicher Eingriffe zu schließen, wurde bereits frühzeitig betont.3) Dabei war der wesentliche kritikpunkt an der finanzwissenschaftlichen Tradition, daB sie die Konsequenzen der Verhaltensannahme eigennützigen Handelns, das sie für das Operieren der Individuen auf den Märkten unterstellte, nicht auch als Grundlage für ein statliches Handeln übernahm.4)

i) vgl. RECKTENWALD (1980), PEACOCK (1980), WOLF (1979)

2) $v$ gl. Z.B. BATOR (1958)

3) BUCHANAN (1962), DAVIS/WHINSTON (1967)

4) ARANSON/FERROR/SASSONE (1978) 
Die meisten Ansätze, soweit sie die Effizienz oder Ineffizienz des States als Gegenstand behandeln, beschäftigen sich hauptsächlich mit den normativen und positiven Problemen einer Ableitung der optimalen GröBe des Staatshaushalts, der Staatsquote.1) Zu fragen ist, inwieweit die Statsquote iberhaupt ein richtiger Indikator für statiche Ineffizienz darstellt und deshalb zum Ausgangspunkt einer Reform genommen werden kann.

Eine Theorie des Staatsversagens muB dabei zwei Ansatzpunkte berücksichtigen: Zum einen kann das Ergebnis des politischen Prozesses ineffizient sein, d.h. nicht mit den Präferenzen der Büger iubereinstimmen. Ein solches Statsversagen soll als situations- oder ergebnisbezogenes Staatsversagen bezeichnet werden.

Daneben kann aber auch der ProzeB der staatlichen Entscheidungen, unabhängig vom Ergebnis dieses Prozesses selbst, ineffizient sein. Ein solches Phänomen soll dann als verfahrensmäßiges oder prozessuales staatsversagen bezeichnet werden.

\subsubsection{Zur Problematik des prozessualen Staatsversagens}

Ebenso wie sich die Theorie der marktmäßigen Allokation mit den Eigenschaften von Zuständen befaßt, die unter verschiedenen Marktformen zustande kommen, liegt auch der Augenmerk der Theorie der statichen Allokation auf den

\footnotetext{
1) Obersicht: AMACHER/TOLLINSON/WILLET (1975), ARANSON/ORDESHOOK (1981), MUSGRAVE (1981), LINDSAY/NORMAN (1977)
} 
Ergebnissen staatlichen Handelns. Dabei wird der ProzeB, wie es zu diesen Zuständen überhaupt kommt, meist vernachlässigt. 1)

Eine Theorie des prozessualen Staatsversagens miß te gerade die Kosten der Entscheidungsfindung in den Mittelpunkt der Analyse stellen, ein Gedanke, der neuerdings von den Theoretikern der "Rent-seeking-society"2) aufgenommen wird.

Dabei läBt sich eine Brücke zu den Oberlegungen BUCHANAN/TULLOCKS (1962) schlagen, die als Grundlage einer Ableitung einer optimalen Entscheidungsregel gerade die Kosten des Entscheidungsprozesses im Auge haben.

BUCHANAN/TULLOCK befassen sich mit dem Problem der optimalen kollektiven Entscheidungsregel. Ihre Oberlegungen gehen dahin, wie groß der Grad der Zustimmung zu einer bestimmten Maßnahme sein muß, d.h. Wie hoch der Anteil der für diese Maßnahme stimmenden Individuen sein muß, damit diese beschlossen und durchgeführt wird. Dabei reicht die Skala von Einstimmigkeit, bei der alle Mitglieder der betrachteten Gruppe einer Maßnahme zustimmen müssen, bis zur allseitigen Diktatur, bei der die Zustimmung eines

\footnotetext{
1) Eine berühmte Ausnahme stellt HAYEK (1945) dar, der die oberlegenheit einer marktwirtschaftichen Ordnung gegenüber einer Planwirtschaft gerade damit begründet, daB der Markt ein viel billigeres und schnelleres Instrumentarium sei, um auf bestimmte Veränderungen zu reagieren, also gerade der ProzeB des Obergangs von einer Gleichgewichtssituation $z u$ einer anderen die Oberlegenheit des Marktsystems demonstriere.
}

2) Obersicht bei TOLLISON (1982) 
einzelnen ausreicht, um diese Maßnahme durchzuführen.1) BUCHANAN/TULLOCK unterscheiden dabei $z$ wei verschiedene Arten von Kosten: Zum einen die externen Kosten einer Entscheidungsregel, d.h. die Nachteile, die für die Mitglieder der Gesellschaft entstehen, die überstimmt werden. Diese externen Kosten sind bei Einstimmigkeit nicht gegeben, da jedes Individuum quasi ein Vetorecht gegen eine Maßnahme hat, die eigenen Interessen entgegenläuft, und sie wachsen mit abnehmender Höhe des zur Entscheidung notwendigen Stimmenanteils. Betrachten wir ein Individuum $i$ und bezeichnen wir die von diesem Individuum erwarteten externen Kosten mit $C_{j}$, so gilt:

$C_{j}=C_{j}\left(N_{a}\right)$

$\frac{d C_{i}}{d N_{a}}<0, \quad N_{a} \leq N, \quad C_{i}(N)=0$

wobe $\mathrm{i} \mathrm{N}_{\mathrm{a}}$ die $\mathrm{Zahl}$ derjenigen Mitglieder der Gesellschaft bezeichnet, deren Zustimmung einer Entscheidung Gültigkeit verleiht, N die Anzahl der Individuen insgesamt symbolisiert.

Als zweite Kostenart bezeichnen BUCHANAN/TULLOCK die sogenannten Entscheidungskosten. Diese Entscheidungskosten kommen dadurch zustande, $d a B$, um zu einer Obereinstimmung zu kommen, die einzelnen Individuen auf irgendeine Weise zusammenwirken und miteinander kooperieren müssen. Das Herbeiführen von Entscheidungen ist selbst kein kostenloser ProzeB. Dabei steigen diese Kooperationskosten mit der Anzahl der für eine Entscheidung notwendigen Stimmen,

1) Aus anderen oberlegungen läBt sich allerdings nachweisen, daß die Mindestanzahl der einer Maßnahme zustimmenden Mitglieder $50 \%+1$ sein muB, da es bei geringerer Zustimmung zu widersprüchlichen Ergebnissen kommen kann. 
am höchsten sind sie bei Einstimmigkeit, wo sie möglicherweise sogar prohibitiv wirken, d.h. so hoch sind, daB es gar nicht mehr zu einer kollektiven Entscheidung kommt. Für das einzelne Individuum stellen sich diese Entscheidungskosten $D_{j}$ dar als:

$D_{i}=D_{i}\left(N_{a}\right)$

$\frac{d D_{i}}{d N_{a}}>0, \quad N_{a} \leq N$

Die für das einzelne Mitglied optimale Regel über die zu einer Entscheidung notwendige Mehrheit ergibt sich dann als die, bei der die Summe aus (erwarteten) externen Kosten und Entscheidungskosten minimiert wird, d.h. wo die Grenzkosten der Entscheidungsfindung gleich den marginalen externen kosten sind.

Dabei interessieren in diesem Zusammenhang gerade die Entscheidungskosten. Im Modell BUCHANAN/TULLOCK's waren diese Entscheidungskosten allein von der Höhe der zu einer Beschlubfassung notwendigen Stimmen abhängig, eine Annahme, die auf der Voraussetzung einer gegebenen institutionellen Ausgestaltung des Entscheidungsprozesses beruhte. Oberlegungen zu einem prozessualen Staatsversagen sollen hier von einer gegebenen Entscheidungsregel ausgehen und untersuchen, inwieweit eine veränderte institutionelle Ausgestaltung des Entscheidungsprozesses (ohne Veränderung der Regel selbst) zu einer Veränderung der Entscheidungskostenfunktion führen kann.

Die Grundidee ist, $d a B$ es unter jeder Entscheidungsregel, die eine bestimmte Stimmenzahl als Grundlage eines Beschlusses festlegt, eine Minimal(entscheidungs-)kostenkombination gibt. Diese Minimalkosten müssen mit den tatsächlichen im Entscheidungsprozeß auftretenden Kosten 
verglichen werden. Ergibt sich dann, daß die tatsächlich zur Beschlußfassung aufgewandten Kosten höher sind als die minimalen Kosten, so soll von einem prozebbezogenen Statsversagen gesprochen werden.

Man könnte von einer X-Ineffizienzll) des Entscheidungsprozesses sprechen: Es werden mehr Ressourcen zur Produktion bestimmter Ergebnisse in Anspruch genommen als notwendig, wobei die Ressourcen allein fiur die Herbeiführung des Beschlusses, nicht für die Ausführung selbst verwandt werden.

Als Beispiele für solche Entscheidungskosten können z.B. der Druck von Wahlzetteln, die Ausgaben der Parteien für Werbung, aber-auch der Aufwand an Zeit und Geld, die der einzelne Bürger zur Beteiligung am politischen ProzeB in Parteiarbeit, Burgerinitiativen, zum Studium politischer Informationen, zur Teilnahme an Demonstrationen usw. aufwendet, genannt werden. Ebenso dazu gehören Zahlungen an Abgeordnete, Regierungsmitglieder, wissenschaft liche Stäbe, aber auch an Interessengruppen usw., kurzum alles, was an einer Entscheidungsvorbereitung beteiligt ist.

Dabei findet nur ein Teil dieser Entscheidungskosten im staatlichen Budget seinen Niederschlag, ein GroBteil dieser kosten dirfte privat anfallen und zu keinen

1) Vgl. LEIBENSTEIN (1966). Dieser entwickelte den Begriff "X-Ineffizienz" im Zusammenhang mit einer Kritik an einer Betrachtungsweise, die Wohlfahrtsverluste aus der Existenz eines Monopols nur aus dem gegenüber dem Konkurrenzfall veränderten Preis-/Mengenverhältnis im Gleichgewicht ableitete. Wichtiger als eine mögliche "allokative" Ineffizienz, die sich aus monopolistischem Verhalten ergibt, ist nach LEIBENSTEIN die "X-Ineffizienz", die im wesentlichen darauf zurückzuführen ist, daß im Monopolfall nicht zu den technisch möglichen Minimalkosten produziert wird. Nach LEIBENSTEIN steigen mit zunehmendem Konzentrationsgrad die Produktions kosten, verursacht durch mangelnden Zwang zur Kostendisziplin und wachsender Irrationalität. 
entsprechenden staatlichen Ausgaben führen. Die Größe des öffentlichen Sektors ist entsprechend kein eindeutiges Indiz für die Höhe der Entscheidungskosten, ein Wachstum oder ein Sinken des Statsanteils muß nichts mit steigenden oder sinkenden Entscheidungskosten zu tun haben.

2.1.2 Zur Problematik des situationsbezogenen Staatsversagens

\subsubsection{Der Begriff der Belastung durch den Staat}

Im allgemeinen wird, wenn von Staatsversagen die Rede ist, der Begriff mit einem Angriff auf die Höne der Staatsquote verbunden. Die Staatsquote stellt dabei den quantitativen Ausdruck des Anteils der dem privaten Sektor entzogenen Ressourcen dar. Allerdings ist der Stat kein Monstrum, das dem privaten Sektor Ressourcen entzieht und diese dann verschluckt, offensichtlich werden diese Ressourcen (transformiert oder nichttransformiert) wieder an den privaten Sektor abgegeben.

Stellen wir uns auf den Standpunkt der VGR: Demnach wird der statliche Output, die Leistungsabgabe des States an die Privaten, immer mit dem Gesamtwert der Inputs bewertet. Demnach spielt die Höhe der Staatsquote für die Lage der Privaten insgesamt keine Rolle: eine höhere Staatsquote heiBt $z$ war, daB den Privaten mehr an Ressourcen entzogen wird, aber eben diese Menge an Ressourcen wird innen in der einen oder anderen Form wieder über die Staatsausgaben zur Verfügung gestellt. $D a B$ diejenigen, die die Ressourcen aufbringen, nicht identisch sind mit denjenigen, denen diese Ressourcen nachher durch den Stat zur Verfügung gestellt werden, mag sein, ist aber für den hier untersuchten Zusammenhang irrelevant. Der Grund für eine generelle Unzufriedenheit mit der Höhe der Staats- 
quote muB woanders liegen: Offensichtlich ist die Bewertung der volkswirtschaftlichen Gesamtrechnung falsch: Es ist davon auszugehen, $d a B$ der Wert der den Privaten abverlangten Leistungen höher ist als in der VGR ausgewiesen, und/oder der Wert des statichen Outputs nicht dem in der VGR berechneten entspricht. Dieser Zusammenhang soll im folgenden deutlich gemacht werden.

\section{A. Der Wert der Steuern}

Angesprochen ist die Problematik, die in der Finanzwissenschaft unter dem Begriff der "wahren Steuerbelastung" diskutiert und gerade in der jüngsten Zeit in der "optimal-taxation"-Literatur in den Mittelpunkt der Analyse geriickt wurde.1)

Steuern beinhalten offensichtlich einen Entzug an potentieller Verfügungsgewalt über Sozialprodukt (der nicht unbedingt den Steuerzahler treffen muß, wenn diesem eine Oberwälzung gelingt). Neben den dabei direkt abzuführenden Beträgen entstehen dem Steuerpflichtigen naturlich noch weitere Kosten, die mit der Prozedur der Berechnung und Abführung der Steuer zusammenhängen (compliance costs) und je nach Steuerart variieren können. Diese stellen natürlich ebenso eine Belastung dar wie die Steuern selbst. Solche Kosten müssen ebenso mitberechnet werden wie die Steuerzahlungen selbst.

Wichtiger erscheinen aber die Kosten, die nicht als direkte, "monetäre" Reduktion des Befriedigungsniveaus ausgedrückt sind: die sogenannte Mehrbelastung der Wirtschaftssubjekte, die am Beispiel einer speziellen Verbrauchsteuer dargestellt werden kann.2)

i) vgl. Z.B. v. OEHSEN (1982)

2) vgl. MUSGRAVE (1969) S. $113 \mathrm{ff}$. 
Folgende Zeichnung stellt diesen Zusammenhang dar:

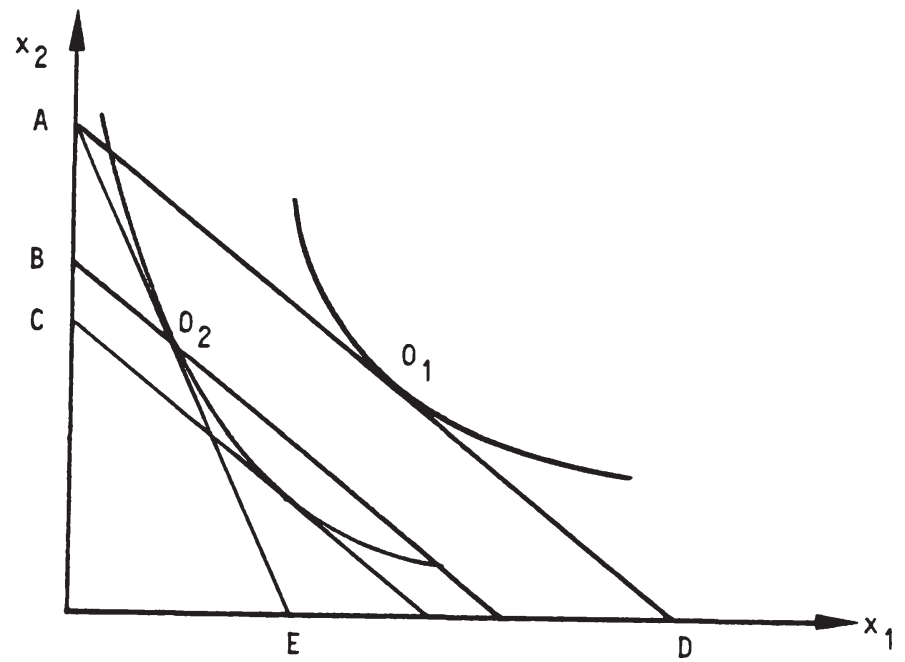

Seien $x_{1}$ und $x_{2}$ zwei Güter (wobei $x_{2}$ auch für ein Güterbündel mit konstanten relativen Preisen stehen kann) und werde $x_{1}$ mit einer spezifischen Verbrauchsteuer belegt. Dies bedeutet eine Drehung der Budgetgeraden von AD nach $A E$, die wirtschaftssubjekte realisieren als neuen Optimalpunkt $\mathrm{O}_{2}$, das in $x_{2}$ ausgedrückte steueraufkommen beträgt $A B$. Die in $x_{2}$ ausgedrückte Belastung des Wirtschaftssubjekts ist aber gröBer: Diese ist so zu errechnen, daB man das Einkommen (in $x_{2}$ ausgedrückt) zum Maßstab nimmt, das das betrachtete Individuum für eine Aufhebung der speziellen Steuer zu zahlen bereit ist. Dies ist durch eine Parallelverschiebung der Budgetgeraden zu ermitteln, bis diese die neue Indifferenzkurve tangiert. Im betrachteten $\mathrm{Fall}_{1}$ beträgt diese EinkommensgröBe ( $i{ } x_{2}$ ausgedruckt) $A C$, es entsteht somit eine Mehrbelastung in Höhe von $B C$ Einheiten.

Auch wenn die Ergebnisse dieses partialanalytischen Modells nicht ohne weiteres auf ein Totalmodell ubertragen 
werden können, läßt sich festhalten: Jede Besteuerung, die nicht allgemein ist in dem Sinne, daß jede mögliche Aktivität mit dem gleichen marginalen Steuersatz belegt wird, oder die an (marginal) preisunabhängigen Aktivitäten ansetzt, fuhrt zu einem Wohlfahrtsverlust, der über dem erzielten Steuerbetrag liegt. Der Wert der Steuern ist somit höher als das, was an Steuereinnahmen ausgewiesen wird.

Diese für eine spezielle Verbrauchsteuer abgeleiteten Ergebnisse gelten natürlich genauso für eine allgemeine Verbrauchsteuer oder eine Einkommensteuer. In diesem letzten Fall werden Freizeitaktivitäten nicht von der Steuer erfaBt und zukünftiger Konsum sogar doppelt. Auch Abwanderungen in die sogenannte "Schattenwirtschaft" mögen eine Rolle spielen, wobei dies allerdings nur dann zu Effizienzverlusten führt, wenn die "schwarz" eingesetzten Faktoren im formalen Sektor einen höheren Ertrag hätten erzielen können.

\section{B. Der Wert der öffentlichen Leistungen}

a) Rein öffentliche Güter

Wurde bei der Bewertung der Steuern an der individuellen Zahlungsbereitschaft angeknüpft, kann bei öffentlichen Leistungen ebenso vorgegangen werden. Dabei soll als erstes eine Bewertung rein öffentlicher Güter versucht werden.1)

1) Als öffentiohe Güter sollen im AnschluB an SAMUELSON (1954) solche behandelt werden, deren Konsum nicht rivalisiert, d.h. die von allen in gleicher Menge konsumiert werden. Generalisierend kann das vorgeführte Konzept aber auch für alle Güter angewandt werden, die mit externen Effekten der Konsumption verbunden sind. In diesem Fall ist nicht mehr das Gut als solches, sondern der Konsum des Gutes durch ein spezifisches Individuum "öffentlich". 
Dabei sind verschiedene Maßstäbe zur Ermittlung der individuellen Zahlungsbereitschaft als Wertma $B$ von öffentlichen Gütern möglich. Am eingängigsten dürfte wohl der Ansatz sein, analog zum Verfahren der Marktpreisbildung die marginale Zahlungsbereitschaft zum Maßstab zu nehmen.1)

Folgendes Bild soll die Sachlage verdeutlichen: Auf der Abszisse soll die Menge der bereitgestellten öffentlichen Güter abgetragen werden, auf der Ordinate sei die Höhe der marginalen individuellen $Z$ ahlungsbereitschaft abgetragen. Es läßt sich dann eine "Pseudo"-Nachfragekurve konstruieren, die für jedes Individuum (hier: 1,2) die jeweilige marginale Zahlungsbereitschaft bei jeder Menge des öffentlichen Gutes darstellt. Die Gesamtnachfragekurve nach öffentlichen Guitern ergibt sich dann durch die vertikale Addition der individuellen Zahlungsbereitschaf ten. Diese stellen dann eine Art Pseudopreis für die öffentlichen Güter dar, der unabhängig von den Kosten der Bereitstellung des öffentlichen Gutes ermittelt werden kann.

i) dazU AARON/MCGUIRE (1970) 


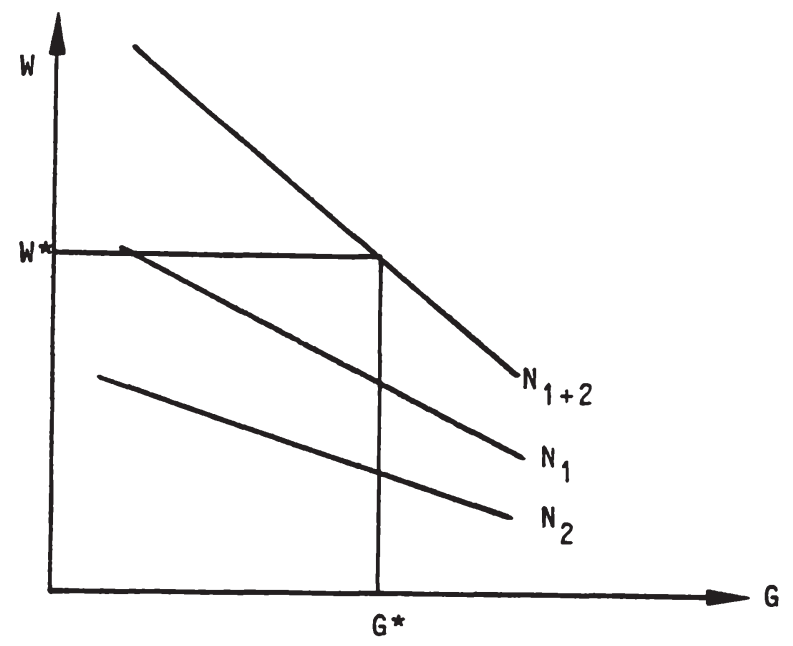

b) Offentliche Leistungen, die nicht-öffentliche Güter sind

Neben diesen rein öffentlichen Gutern stellt der Staat auch dem Charakter nach private Güter bereit. Auch in diesem Fall läBt sich als BewertungsmaBstab die (marginale) Zahlungsbereitschaft der NutznieBer dieser statich bereitgestellten Güter heranziehen.

Im einfachsten Fall stellt der Staat jedem einzelnen genau die Menge an privaten Gütern zur Verfügung, die der Konsument auch ohne staatliches Eingreifen zu den dann herrschenden Marktpreisen gekauft hätte. In diesem Fall ist eine Marktpreisbewertung der staatlichen Leistungen zutreffend und angebracht. So die Kostenstruktur der statichen Güterbereitstellung nicht von den (hypothe- 
tischen) Kosten der privaten Bereitstellung abweicht, füht auch eine Bewertung zu (Grenz-)kosten zu dem gleichen Ergebnis.1) Für den Fall, daß der Staat nur einen Teil der Kosten der privaten Güter übernimmt, deren Konsum also subventioniert, könnte die Differenz zwischen Marktpreis und von den Konsumenten bezahltem Preis als Wertmaßstab herangezogen werden.

Allerdings durfte eine Obereinstimmung der vom Stat zur Verfügung gestellten Menge mit derjenigen, die ohne staatliche Aktivität am Markt produziert und von jedem einzelnen Konsumenten nachgefragt worden wäre, selten vorkommen. Dann aber ist die Bewertung komplizierter, da der den privaten Gütern von den Individuen zugerechnete Wert nicht mehr mit dem Marktpreis ubereinstimmt. Das soll im folgenden verdeutlicht werden.

\section{1) Vollständige Subventionierung}

Unter vollständiger Subventionierung soll hierbei der Fall verstanden sein, in dem bestimmte private Güter vom stat unentgeltich abgegeben werden, bzw. die den Privaten entstehenden Kosten $z u$ 100\% ubernommen werden, also keine Eigenbeteiligung erforderlich ist. Die Privaten werden diese Leistungen dann bis zur Sättigungsmenge nachfragen. zeichnen wir ein Indifferenzkurven-Diagramm, ergibt sich folgendes Bild:

1) Das setzt naturlich voraus, daB am Markt die Grenzkosten-Preis-Regel gilt, d.h. die Preise z.B. nicht aufgrund monopolistischer Angebotsstruktur ïber den Grenzkosten liegen. 


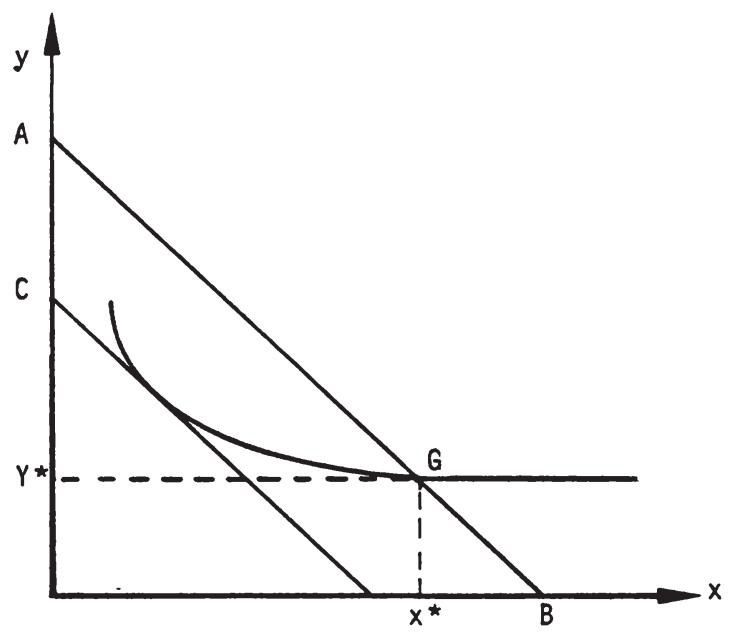

y bezeichne dabei das private Einkommen, $x$ die öffentliche Leistung.

Eine Sättigungsmenge von $x^{\star}$ ist dann gegeben, wenn die Indifferenzkurve parallel zur $x$-Achse läuft, das heiBt, eine Erhöhung von $x$ führt für das jeweilige Individuum nicht mehr zu einer Situation, die es vorzieht. Bei gegebenem Einkommen $y^{*}$ wird dann als Optimum der Punkt G angenommen.

Die Produktionskosten von $x$ sollen durch die Steigung der Geraden $A B$ bestimmt werden (Annahme: konstante Grenzkosten). Nach Kostenbewertung wäre dann der Wert der dem Haushalt zufließenden staatlichen Leistungen gleich $y * A$, der dem Haushalt tatsächlich zuzuschreibende Einkommenswert entspricht aber nur der Strecke $y^{\star} C$, dem Wert des 
Einkommens, das dem Haushalt gezahlt werden müßte, um ihn in eine Lage zu bringen, die er für genauso gut hält wie die Situation $G$.

Einen Spezialfall stellt die Situation dar, in der die statliche Leistung auf ein bestimmtes Niveau begrenzt ist, z.B. im Rahmen einer Sicherstellung des Grundbedarfs nur eine bestimmte Höchstmenge der statiohen Leistung unentgeltiich bereitgestellt wird. Dies läBt sich graphisch folgendermaßen darstellen:

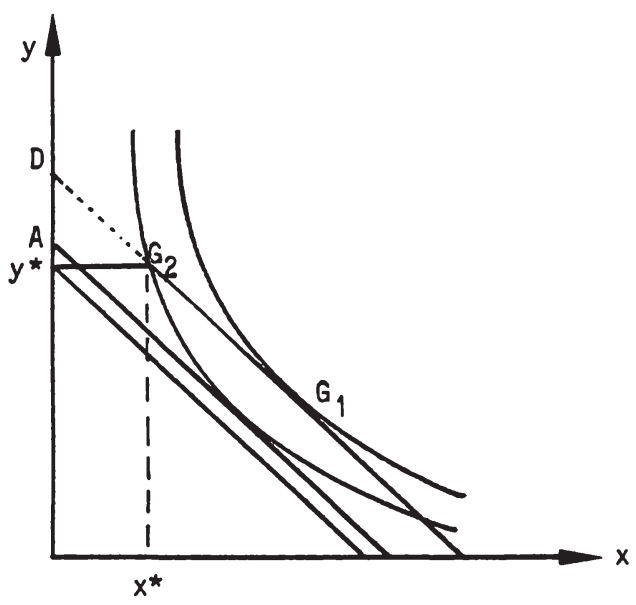

Der Stat stellt eine bestimmte Menge des Gutes $x *$ als Grundsicherung unentgeltiich zur Verfügung. Zwei mögliche Reaktionen des Haushaltes sind dann denkbar: Der Haushalt konsumiert insgesamt mehr dieses Gutes $x$ als vom Stat 
bereitgestellt wird (in der Zeichnung $G_{1}$ ). Dann erst ist es berechtigt, dem Haushalt die gesamten Kosten der Erstellung dieser Leistung als Einkommenswert zuzurechnen.

Oder der Haushalt realisiert die Ecklösung (in der Zeichnung $G_{2}$ ). Dann liegt der tatsächliche Wert der staatlichen Leistung bei $A y^{*}$, nicht bei Dy*. In diesem Fall würde eine Kostenbewertung eine oberschätzung des Wertes der öffentlichen Leistungen für den empfangenen Haushalt bedeuten.

2) Teilsubventionierung

Hier ist der Fall angesprochen, bei dem der Stat nur einen Teil der Kosten der Bereitstellung übernimmt, beziehungsweise die Privaten zur Selbstbeteiligung herangezogen werden. Die staatliche Leistung ist dementsprechend aktivitätsabhängig, zudem muß der einzelne Haushalt aber auch eigene Leistungen aufbringen. Der Fall läßt sich analog zum beschriebenen Steuerfall behandeln: anstelle der Steuer auf eine bestimmte Aktivität kommt es jetzt zu einer Subventionierung. Auch in diesem Fall ist der Wert der Subventionen für den Haushalt kleiner als die im öffentlichen Haushalt ausgewiesenen Mittel.

Insgesamt läßt sich also zeigen, daB der Wert der vom Staat in Anspruch genommenen privaten Ressourcen größer, der vom Staat abgegebenen privaten Güter $k l e i n e r$ ist als der nach der volkswirtschaftlichen Gesamtrechnung ausgewiesene. Bei öffentlichen Guitern liegt die Sachlage etwas anders: hier kann der Wert sowohl über als auch unter den Produktionskosten liegen, je nachdem, ob die angebotene Menge des öffentlichen Gutes $z u k l e i n$ oder $z u$ grob ist. 
Allerdings drückt sich auch hier diese Diskrepanz nicht in der Statsquote selbst aus. Es sind ja gerade die $A b$ weichungen der individuellen Bewertungen der Staatseinnahmen/-ausgaben von den in der VGR ausgewiesenen, die in diesem Zusammenhang problematisch sind.

\subsubsection{Eine Definition situationsbezogenen Staatsver- sagens}

Bis jetzt wurde abgeleitet, wie die Höhe der Belastung durch den Stat zu ermitteln wäre: Sie entspricht der Diskrepanz zwischen dem Wert der öffentlichen Leistungen und dem der staatlichen Einnahmen, wobei die individuellen Zahlungsbereitschaften als Maßstab genommen werden sollen.1)

Als Kriterium fur ein Statsversagen kann dabei an diesen individuellen Zahlungsbereitschaften angeknüpt werden. Dabei soll weniger die Gesamtzahlungsbereitschaft, sondern die marginale Zahlungsbereitschaft der Individuen in den Mittelpunkt gestellt werden. Es gelte: Statsversagen liegt immer dann vor, wenn eine marginale Umstrukturierung des staatshaushalts mit einer positiven individuelien Zahlungsbereitschaft eines jeden verbunden ist.

Diese Veränderung kann durch eine Vielzahl von Maßnahmen erreicht werden, sei es durch eine Erhöhung der Staatsausgaben bei gleichzeitiger Erhöhung der Einnahmen, eine Reduktion beider GröBen, oder eine Umstrukturierung bei konstantem Umfang des Budgets, wie es z.B. bei den Steuereinnahmen von den Theoretikern der "Optimal Taxation" untersucht wird.

1) Für diese Definition vgl. MELTZER/RICHARD (1981) S. 915, BRETON (1974) S. 19 
Aber auch, wenn die marginale, private Zahlungsbereitschaft nicht unter den marginalen Kosten der Leistungserstellung durch den öffentlichen Sektor liegt, kann ein Staatsversagen vorliegen. Dies ist dann gegeben, wenn die öffentliche Produktion nicht zu Minimalkosten stattfindet, d.h. die Art der Leistungserstellung ineffizient ist. Auch hier wäre durch eine Umorganisation der statichen Leistungserstellung ein Gewinn für die Individuen möglich, der allerdings nicht von einer Veränderung der Struktur bzw. Größe des outputs ausginge, sondern durch eine Verbesserung der Effizienz der öffentlichen Leistungserstellung (X-Effizienz).1)

Das hier entwickelte Kriterium entspricht dabei dem Pareto-Kriterium, nach dem eine Maßnahme dann vorzunehmen ist, wenn dadurch mindestens eine Person besser gestellt wird, ohne daB sich eine andere verschlechtert. Damit wird also für die Beurteilung eines Statsversagens vorausgesetzt, daß es zu keiner Umverteilung kommt.

Die Einschränkung, daB Staatsversagen nur für den Fall festgestellt werden kann, wo es nur Gewinner bzw. nur Verlierer gibt, ist allerdings gravierend, da eine solche Situation faktisch so gut wie niemals vorkommen wird. Andererseits können Verteilungswirkungen auch nicht völlig ignoriert werden, indem man die Zahlungsbereitschaften aller Individuen aufsummiert und eine allokative Verbesserung immer dann konstatiert, wenn diese Summe positiv ist, unabhängig wie sich Gewinne und Verluste auf die einzelnen Bürger verteilen. Ein GroBteil der staatichen Maßnahme ist ja auf Umverteilung als Ziel gerichtet. Daher müssen auch die Verteilungsänderungen, die mit einer statichen Aktion verbunden sind, einer Beurteilung

i) Eine ausfiuhrliche Diskussion mangelnder X-Effizienz als Grund für statiche Unwirtschaftichkeit findet sich be i ROSENSCHON (1980) 
unterzogen werden. Einfach wäre dies, wenn es ein vom politischen Prozeß unabhängig zu konstatierendes Verteilungsziel gäbe und die damit verbundene verteilung zum MaBstab der Beurteilung statichen Handelns gemacht werden könnte. Es gibt unter den zahlreichen Ansätzen einer normativen Theorie der gerechten Verteilung jedoch keinen Ansatz, der nicht umstritten ist. Ein allgemeingiiltiges Verteilungskriterium existiert nicht.

Hier sei folgende Lösung vorgeschlagen: Zur Beurteilung von Verteilungsfragen soll wieder auf die gegebene Entscheidungsregel zurückgegriffen werden. Damit soll jeder Verteilungsvorschlag, der unter einer gegebenen Entscheidungsregel gewählt wird, als gleichwertig angesehen werden. Als allokativ vorteilhaft soll eine Maßnahme bezeichnet werden, bei der die aufsummierte Zahlungsbereitschaft der Individuen positiv bzw. bei mehreren Alternativen am größten ist.

Allokative Veränderungen, die mit Verteilungsänderungen einhergehen, können somit dem Test eines Wahlverfahrens unterzogen werden, um festzustellen, ob es sich wirklich um eine "Verbesserung" handelt oder nicht. Sanktioniert der bestehende Wahlmechanismus die Veränderung, so soll sie auch als "besser" angesehen werden. Als Entscheidungsregel wird dabei im folgenden die einfache Mehrheitsregel gesetzt, d.h. die Alternative gilt als besser, der $50 \%+1$ der Entscheidungsberechtigten zustimmen. 1)

Der somit bestimmte Beurteilungsmechanismus hat zwei getrennte Kriterien, die zusammengezogen werden müssen, um ein Staatsversagen zu konstatieren. Eine Situation ist immer dann schlechter als eine andere, wenn bei der

1) Zur Normativen Theorie der Entscheidungsregel siehe TAYLOR (1969), RAE (1969), Obersicht bei MUELLER (1979) 
zweiten die (Netto)zahlungsbereitschaft der Privaten größer ist als bei der ersten und die Verteilungsänderungen durch Mehrheitsbeschluß sanktioniert werden.

Von LITTLE (1950) kommt der Vorschlag, eine Maßnahme dann zu befirworten, wenn 1. diejenigen, die bei dieser Veränderung gewinnen, die Verlierer kompensieren können, bzw. von den Verlierern nicht für den Verzicht auf diese Maßnahme entschädigt werden können, und 2. die Verteilungswirkungen "giunstig" sind.

Der erste Teil des Kriteriums entspricht dem KALDOR/HICKSKriterium, das vor allem wegen der Vernachlässigung der Verteilungsseite kritisiert wurde. Der zweite Teil des Kriteriums versucht diesem Kritikpunkt zu begegnen, allerdings blieb bei LITTLE offen, was unter "giunstigen" Verteilungswirkungen zu verstehen ist.1) Diese Leerstelle soll hier durch den Rekurs auf einen gegebenen Entscheidungsmechanismus gefüllt werden: Verteilungsmäßig günstig ist, was nach der bestehenden Entscheidungsregel, also durch Mehrheitsbeschluß, legitimiert wird.

Reine Paretoverbesserungen werden durch dieses Kriterium auf jeden Fall abgedeckt - die Summe der Zahlungsbereit schaften ist positiv und es wird keiner schlechter gestellt, so daß sich auch keine Gegenstimmen erheben werden. Damit ist aber die Menge der Situationen, die beurteilt werden können, nicht erschöpft. Auch Maßnahmen, die keine reinen Paretoverbesserungen darstellen, da es sowohl Verlierer als auch Gewinner gibt, entziehen sich damit nicht unbedingt einer Beurteilung: findet diese Verteilungsänderung eine Mehrheit, so soll sie nach obigem Kriterium ebenfalls als Verbesserung angesehen werden.

1) Zu der Diskussion um die sog. Kompensationskriterien vgl. SOHMEN (1976), Kapitel 9 
Es ist allerdings nicht anzunehmen, daß eine auf diesem Kriterium beruhende Wohlfahrtsfunktion den Postulaten, die üblicherweise an sie gestellt werden, entspricht: Sie durfte weder dem Kriterium der Vollständigkeit noch dem der Konsistenz (Transitivität) genügen.

Vollstänig ist sie schon deshalb nicht, da eine situation, die zwar gegenüber einer anderen eine positive Zahlungsbereitschaft aufweist, von der Mehrheit aber abgelehnt wird (aufgrund der Verteilungsänderung), nach diesem Kriterium nicht beurteilt werden kann. Nicht alle Situationen können deshalb nach diesem Kriterium einer Beurteilung unterzogen werden. Konsistenz ist ebenfalls nicht zu erwarten: Beide Teile des Kriteriums können durchaus zu widersprüchlichen Aussagen führen.

$D a B$ ein reiner Vergleich von Zahlungsbereitschaften zu widersprüchlichen Ergebnissen führen kann, hat bereits SCITOVSKY nachgewiesen.1) Aber auch der zweite Teil des Kriteriums führt nicht zu konsistenten Ergebnissen: ARROW (1962) wies auf die Inkonsistenz von Mehrheitsentscheidungen hin, und gerade bei Verteilungsfragen durften die in der Literatur erwähnten Voraussetzungen, die eine konsistente Entscheidung garantieren, eben nicht gewährleistet sein.2)

Das Ergebnis ist daher im HöchstmaB unbefriedigend -andererseits durfte es wohl kaum ein kriterium geben, das den logischen Ansprüchen genügt und dennoch von einiger Relevanz für tatsächliche Entscheidungen bleibt.

Angesichts der Schwierigkeiten, vor denen die Suche nach einem den Anforderungen der Konsistenz und Vollständigkeit geniugenden Kriterium zur Beurteilung verschiedener

i) Vgl. SCITOVSKY (1941/42)

2) vgl. dazu später Abschnitt 3.3.2. 
Situationen steht, wenn es mehr als eine extrem eingeschränkte Gültigkeit beanspruchen will, lassen sich diese Postulate nicht aufrechterhalten. Da zu hohe Anforderungen an ein Entscheidungskriterium letztlich dazu fiuhren, daB die Klasse der damit zu beurteilenden Entscheidungen sehr klein wird, wird letztendlich nur der Weg übrigbleiben, die Inkonsistenz einer Regel, wie sie eben besprochen wurde, zu akzeptieren. Wenn die Möglichkeit und faktische Existenz von Widersprüchlichkeiten zu konstatieren ist, dann ist es eventuell besser, diese als Bestandteil der Welt hinzunehmen und nicht das MaB der Logik an sie anzulegen mit dem Ergebnis, $d a B$ man zu den Problemen nichts mehr zu sagen hat.

2.2. Grundiuberlegungen zur Rationalität von Budgetgrenzen als konstitutionelle Regelungen

Im folgenden soll dargestellt werden, welche grundsätzlichen Oberlegungen mit der Idee, Budgetbegrenzungen zur Erhöhung der Effizienz statichen Handelns einzuführen, verbunden sind. Dabei kann die Einführung von Budgetbeschränkungen als eine Methode angesehen werden, um eine "Second-Best"-Lösung zu realisieren.

Bekanntlich geht die Theorie des "second-best"1) von einer Situation aus, in der aufgrund bestimmter, nicht aufhebbarer Beschränkungen (Constraints) ein Gesamtoptimum als "erst-beste" Lösung nicht erreicht werden kann. Die zweitbeste Lösung sieht dann so aus, daß man die optimale

i) dazU LIPSEY/LANCASTER 
Kombination von Abweichungen von der erstbesten Situation zu realisieren sucht. Es geht also um die Suche nach sich gegenseitig kompensierenden Fehlern. Beispiele für die Anwendung der"Second-best"-Theorie liegen z.B. auf dem Gebiet der "Optimal Taxation" oder der Zollunionstheorie. Allerdings setzt auch die Theorie des "second-best" eine Institution voraus, die, wenn sich eine zweitbeste Lösung nicht von selbst ergibt, die dazu notwendigen Maßnahmen durchfuihrt.

Möglicherweise stellt sich aber die Alternative, durch eine Kombination von Maßnahmen eine erst-oder zweitbeste Lösung durchzusetzen, gar nicht, da es keine Instanz gibt, die für die Herbeiführung eines $0 p t i m u m s$ zuständig ist bzw. deren Ziel eine möglichst optimale Lösung darstellt.

Die Einführung von Budgetbeschränkungen ist unter diesem Aspekt zu sehen. Sie wären dann ein Instrument, um den Handlungsspielraum der Entscheidungsträger so zu verengen, daB die Situation, die sich unter diesen Beschränkungen als Ergebnis ihres Handelns ergibt, besser ist als die Lösung, die sich ohne Existenz von Budgetgrenzen ergeben würde. Es geht darum, das Rahmenwerk der Entscheidungen so $z u$ verändern, daß aus den möglichen suboptimalen Lösungen noch die relativ beste gewählt wird. Zu fragen ist, ob Budgetbeschränkungen eine solche wirkung zugeschrieben werden kann.Budgetbeschränkungen haben somit die Funktion von (zusätzlichen) Regelungen, die bestimmte Anforderungen an die Ausgestaltung des Entscheidungsprozesses stellen. Budgetgrenzen legen nicht unmittelbar das Ergebnis des Handelns fest, sondern geben, nur den Rahmen vor, in dem dieses Handeln sich entfalten kann. 
Diese Funktion entspricht der Unterscheidung, die BUCHANAN 1 ) zwischen Entscheidungen auf der konstitutionellen Ebene und auf der nachkonstitutionellen Ebene (operationale Entscheidungen) trifft. Das erstere beinhaltet die Entscheidung uber die Regeln, unter denen man die zweite Art der Entscheidung, die über die Probleme selbst, trifft. Entscheidungen auf Verfassungsebene sollen dabei nach BUCHANAN/TULLOCK prinzipiell einstimmig gefaBt werden. Damit ist gewährleistet, daß nur Verfahren gewählt werden, die von allen akzeptiert werden.

Allerdings dirfte die Forderung nach Einstimmigkeit nicht durchfiuhrbar sein, da die Kosten der Entscheidungsfindung und des Verhandelns dann prohibitiv hoch würden. Tatsächlich gibt es in den realen Verfassungen auch keine Regel, zu deren Änderung Einstimmigkeit Voraussetzung ist. Insgesamt aber durfte der Grad an Zustimmung, der zu einer Formulierung oder Änderung von Verfassungsvorschriften notwendig ist, bei weitem höher liegen als der Grad der Zustimmung, der fur einfache Entscheidungen auf der operationalen Ebene erforderlich ist. Realiter wird eine 2/3-Mehrheit allgemein zur Änderung von Verfassungsvorschriften notwendig sein.

Dabei postuliert BUCHANAN2), daB "faire" Entscheidungen uber die Regeln der kollektiven Wahl Unsicherheit der Mitglieder der Gesellschaft über ihre spätere Lage voraussetzen. Die Idee dabei ist, daB die Entscheidung uber die Regeln, also auf konstitutioneller Ebene, nicht beeinflußt werden soll von der aktuellen oder zuküntigen Lage jedes einzelnen unter der jeweils herrschenden Regel.

\footnotetext{
1) BUCHANAN/TULLOCK (1962), BUCHANAN (1975a) (1975b)

2) und ebenso RAWLS (1971)
} 
Der Einzelne soll seine Entscheidung iber die Ausgestaltung der Gesellschaft treffen, ohne zu wissen, welche Position er selbst darin einnehmen wird.

Nur wenn diese Trennung vollzogen werden kann, ist eine Trennung der Entscheidung auf konstitutioneller Ebene von einer operationalen Entscheidung überhaupt möglich. Kennt nämlich der einzelne Bürger die Lage, die er unter alternativen Entscheidungsregeln einnimmt, so wird er wahrscheinlich diejenige wählen, die ihn persönlich in die bestmögliche Situation bringt. Damit ist das Kalkiil der Entscheidung uiber die Regel beeinfluBt vom Ergebnis der Entscheidungen unter dieser Regel. Eine "faire" Entscheidung uiber den zukünftigen zustand setzt daher Unsicherheit uiber den eigenen Platz, den man letztlich einnimmt, voraus.

Eine derartige Oberlegung ist mit Recht dahingehend kritisiert worden, $d a B$ es den Zustand "unbefleckten Nichtwissens" nicht gibt, d.h. daB die Entscheidungsträger bereits bei der Entscheidungsfindung auf konstitutioneller Ebene Vorstellungen darüber haben, welchen EinfluB die einzelne Regel auf ihre spätere Lage haben wird. Eine "reine" konstitutionelle Entscheidung gibt es demnach nicht.

Dies gilt insbesondere dann, wenn es sich um eine nachträgliche Änderung der Verfassung handelt, wie es hier für die Einführung von Budgetbeschränkungen der Fall ist. Dennoch hat die Unterscheidung für die hier präsentierte Problematik einen Sinn. Auch hier wird argumentiert, daB budgetäre Beschränkungen ähnliche "konstitutionelle" Regeln sind, wie BUCHANAN sie beschreibt. Sie limitieren den Bereich, in dem sich die einzelnen (operationalen) Entscheidungen über die konkrete Ausgestaltung des Budgets bewegen. 
Allerdings kennen die Individuen ihre jetzige Lage und werden auch Vorstellungen darüber haben, wie eine dergeartete Regeländerung sich auf die eigene Situation auswirken durfte. Damit stehen wir vor folgendem Paradox: Da die Bestimmungen zur Änderung der Regel einen wesentlich höheren Zustimmungsgrad erfordern als Entscheidungen auf der operationalen Ebene, ist zu fragen, wieso sich Individuen auf eine Änderung des Entscheidungsrahmens einigen könnten, die ihre Lage verbessert, und gleichzeitig nicht zu demselben Ergebnis durch (weniger anspruchsvolle) operationale Entscheidungen kommen.

Um dieses Paradox aufzuhellen, soll ein spieltheoretisches Modell herangezogen werden.

Die Ergebnisse der operationalen Entscheidung der Spieler $A$ und $B$ in Abhängigkeit von den jeweils gewählten Strategien $a_{i}, b_{i}, i=1,2,3$, sollen sich in folgender Matrix darstellen lassen:

\begin{tabular}{rcccc} 
& & \multicolumn{4}{c}{ Spieler $B$} \\
& & $b_{1}$ & $b_{2}$ & $b_{3}$ \\
& $a_{1}$ & 5.2. & 1.2. & 2.3. \\
Spieler $A$ & $a_{2}$ & 1.1. & 0.0. & 1.2. \\
& $a_{3}$ & 3.3. & 2.1. & 2.2.
\end{tabular}


Die in der Matrix stehenden Ziffern sollen jeweils Zahlungsströme ausdrücken, die bei einer bestimmten Entscheidungskonstellation an die beiden Spieler fließen, wobei die erste $Z a h l$ die Auszahlungen an Spieler $A$, die zweite die an B symbolisiert.

In diesem Fall gibt es keine dominante Strategie, die Spieler dirften wohl eine Mischung aus $a_{1}$ und $a_{3} b_{2} w$. b 1 und b3 wählen. Das schlechtestmögliche Ergebnis würde in diesem Fall eintreten, wenn beide Spieler die Strategie 2 wählen würden. Eine konstitutionelle Vereinbarung könnte jetzt dahingehen, $d a B$ die Wahl dieser Strategie durch einen der Spieler verboten wäre. Diese Entscheidung würde die Zustimmung beider Spieler finden, könnte also einstimmig gefaßt werden. Sie wäre allerdings in diesem Fall überflussig, weil die Strategie 2 auch auf operationaler Ebene nicht gewählt worden wäre.

Anders verhält es sich, wenn wir folgende Auszahlungsmatrix betrachten:

Spieler B

$\begin{array}{ccccc} & & b_{1} & b_{2} & b_{3} \\ & a_{1} & 4.4 & 3.2 . & 0.6 . \\ \text { Spieier A } & a_{2} & 3.7 & 2.6 . & 1.8 . \\ & a_{3} & 5.1 . & 4.1 . & 2.2 .\end{array}$


Diese Situation ist die gleiche, die dem berühten Gefangenen-Dilemma zugrundeliegt: Strategie 3 dominiert fuir beide Spieler, d.h. diese Strategie wird bei unabhängiger Anpassung (Nicht-Kooperation) gewählt werden, und somit mit Sicherheit eine Situation zustande kommen, die möglichen anderen unterlegen ist. Für beide Parteien wäre es unter diesen Umständen günstig, sich auf eine Regel zu einigen, die einen Verzicht auf die Wahl der Strategie 3 beinhaltet.

Diese Regel kann implizit als auch explizit durchgesetzt werden. Eine implizite Vereinbarung wäre dann gegeben, wenn beide Partner von sich aus auf die Wahl der Strategie 3 verzichten, ohne daß es zu einer formalen obereinkunft kommt. Dies wäre eine Vereinbarung auf rein operationaler Ebene, die Wahrscheinlichkeit eines solchen Handelns hängt von verschiedenen Faktoren (wie Höhe des Gewinns bei beiderseitigem kooperierenden Verhalten, Anzahl der Teilnehmer) ab. Allerdings kommen die meisten empirischen Untersuchungen zum Ergebnis, daB die Tendenz zu kooperativem Verhalten immer sehr gefährdet ist. 1 )

Daher bietet sich eine explizite Vereinbarung uiber eine Änderung der Regel des Spieles an, infolge derer Strategie 3 nicht mehr gewählt werden kann. Eine solche Regel kann als Entscheidung auf der konstitutionellen Ebene angesehen werden, da beide Parteien bei dieser Entscheidung über einstimmen muissen und durch diese festlegung nicht auf ein bestimmtes Ergebnis gezielt wird.

Herrscht in dem gewählten Beispiel noch vollkommene GewiBheit über das Ergebnis, das sich nach einer Änderung der Verfassung ergibt, und kann dadurch eine Paretover-

i) $v$ gl. RAPAPORT/CHAMMAH (1965), TAYLOR (1976) 
besserung erreicht werden, so ist dies nicht die einzige Möglichkeit, die zu einer bestimmten option für eine konstitutionelle Regel führen kann.

Eine Einigung ist auch immer dann möglich, wenn der erwartete Nutzen eines Mitgliedes der Gesellschaft nach einer Verfassungsänderung höher ist als ohne diese Änderung. Der Unterschied zu dem ersten Beispiel scheint auf den ersten Blick nur minimal zu sein: Im ersten Fall haben wir es mit sicheren Ergebnissen zu tun, im zweiten Fall mit unsicheren, mit Wahrscheinlichkeiten zu gewichtenden Resultaten. Allerdings führt unter der Voraussetzung der vollkommenen GewiBheit nur eine tatsächlich mögliche Paretoverbesserung zu einer Einigung über die Änderung der Verfassung, während im $F$ all der Unsicherheit Ex-ante- und Ex-post-Ergebnis auseinanderfallen können. Zwar wird der erwartete Nutzen aus einer Verfassungsänderung für alle positiv sein. Im nachhinein mag es aber durchaus Verlierer und Gewinner geben.1) $D a B$ aber die Entscheidung für eine Verfassungsänderung aus der Expost-Sicht falsch war, es also letztendlich nicht zu einer Paretoverbesserung gekommen ist, spielt für die Entscheidung selbst keine Rolle: Sie ist ja im voraus zu treffen.

Soweit also nicht nur der Fall der reinen (Ex-post- und Ex-ante-)Paretoverbesserung betrachtet werden soll, kann eine Einigung auf eine Verfassungsänderung nur unter der Voraussetzung zustande kommen, $d a \beta$ zumindest ein bestimmter Grad an Unsicherheit über die eigene Lage danach besteht, und dennoch der Erwartungswert der möglichen Nutzenpositionen gröBer ist als der Wert ohne die entsprechenden Verfassungsregeln. Diese Anforderung ist nicht so streng wie die Voraussetzung der vollkommenen Unge-

i) zu dieser Unterscheidung vgl. auch MUELLER (1977) 
wiBheit über die zukünftige eigene Lage, wie sie für eine "faire" Entscheidung auf konstitutioneller Ebene postuliert wurde, weist aber doch bestimmte Parallelen auf.

Zieht man nun eine Verbindung zu den in Abschnitt 2.1. behandelten Definitionen des Staatsversagens, so ergibt sich folgender Zusammenhang: Die Einfiuhrung einer Budgetbeschränkung $1 a ̈ B t$ die eigentliche Entscheidungsregel, in diesem Fall die Mehrheitsregel, unberührt. Lassen sich

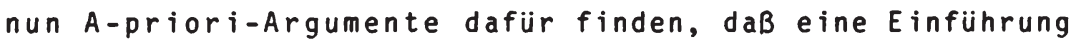
von Budgetgrenzen zu einer Verringerung des wie oben definierten statsversagens führt, so ergeben sich auch Punkte, die es als wahrscheinlich erachten lassen, daB diese Verfassungsänderung auch auf Zustimmung trifft und realisiert wird.

Eine Verringerung des Statsversagens bedeutet ja ein Mehr an zur Verfügung stehenden Ressourcen: Die Aufwendungen zur Entscheidungsfindung (prozessuales Statsversagen) oder die bewerteten Effizienzverluste des sich einstellenden Ergebnisses des politischen Prozesses (situationsbezogenes Staatsversagen) werden dabei ja verringert. Andererseits wird über die Verteilung der Gewinne mit derselben Regel beschlossen, die auch für die Verteilung vorher ausschlaggebend war: Die Mehrheitsregel an sich wird ja nicht in Frage gestellt.

Betrachtet man die Lage des einzelnen Individuums, das - immer unter der Voraussetzung, $d a B$ eine Budgetbeschränkung zu einer Verringerung des Statsversagens fuihrt -, Erwartungen iber seine Lage nach der Verfassungsänderung bildet, folgt: Da die Mehrheitsregel als Entscheidungskriterium uber die Verteilung weiterhin erhalten bleibt, diurten seine Erwartungen uber die möglichen spezifischen Verteilungssituationen, in denen es sich mit oder ohne Budgetbegrenzung befindet, nicht wesentlich voneinander 
abweichen. Andererseits ist der Wert der gegebenenfalls zur Verteilung anstehenden Ressourcen höher als im Fall ohne Budgetbeschränkungen, so daß insgesamt aus der Ex-ante-sicht der Erwartungswert des Nutzens aller möglichen Situationen bei einer Verfassungsänderung höher liegt als ohne Budgetgrenze. Daraus folgt, daB das Individuum daher einer Verfassungsänderung zustimmen dirfte.

Im folgenden ist also zu untersuchen, ob es A-priori-Argumente gibt, die darauf schließen lassen, daB die Einführung einer Budgetbegrenzung zu einer Verringerung des Statsversagens führt. Nur wenn dies erfüllt ist, erscheint eine Strategie der verfassungsmäBigen Verankerung solcher Grenzen als sinnvoll und durchsetzbar.

\subsection{Die verschiedenen Formen der Budgetbeschränkungen}

BUCHANAN/BRENNAN u.a.1) unterscheiden in ihrer Taxonomie budgetärer Beschränkungen zwei groBe Komplexe: verfahrensmäßige und ergebnisbezogene Begrenzungen.

\subsubsection{Verfahrensmäßige Begrenzungen}

Hier wird das Verfahren der Aufstellung, des Beschlusses oder der Kontrolle des Budgets bestimmten Regeln unterworfen, die gegenuber der bisherigen Ausgestaltung des Entscheidungsprozesses beziehungsweise des fuir andere statiche Maßnahmen geltenden Regelwerks schärfer und 
restriktiver formuliert sind. Solche Regeln existieren bereits - das Aufstellen eines einheitlichen Haushaltsplanes und die damit in der Verfassung verknüpften Vorschriften sind Regeln, die den BudgetierungsprozeB reglementieren. Diese bereits bestehenden Verfahrensvorschriften können durch andere ergänzt werden. Genannt werden zum Beispiel eine Integration von Einnahme- und Ausgabeentscheidung, die Zweckbindung öffentlicher Einnahmen, spezielle Mehrheiten (beispielsweise zwei Drittel) für eine Steuer- oder Ausgabenentscheidung, usw. Für alle gilt, daB die Höhe des Budgets selbst weiterhin dem 'normalen' politischen Entscheidungsproze $B$ überlassen wird, daß nur die Vorgehensweise innerhalb dieses Prozesses verändert wird. Solche MaBnahmen sollen hier im weiteren nicht untersucht werden.

2.3.2 Ergebnisbezogene oder quantitative Budgetbegrenzungen

Im Gegensatz zu den verfahrensbezogenen Beschränkungen wird hier nicht der EntscheidungsprozeB selbst reglementiert, sondern ein bestimmter, quantitativ fixierter Spielraum vorgegeben, der als A-priori-Restriktion die Ergebnisse der politischen Entscheidung eingrenzt: Die Politiker sind nicht mehr frei, alle möglichen Budgets durchzusetzen, sondern können nur noch in einem bestimmten Rahmen agieren, ohne daB die Art, wie dieser Rahmen ausgefüllt wird, selbst irgendwelchen Restriktionen unterliegt. Zu unterscheiden sind dabei zwei verschiedene Ansätze: Einmal können (nur) bestimmte spezielle Parameter des staatlichen Handelns im voraus fixiert werden, ohne $d a B$ die anderen Parameter einer Reglementierung unterliegen. Zum anderen können globale Größen vorgegeben werden, die sich normalerweise als Ergebnis verschiedener Parame- 
terkonstellationen ergeben, wobei dann die Wahl der Parameter, soweit man sich im vorgegebenen Rahmen bewegt, frei bleibt.

\section{A. Spezielle Budgetgrenzen}

Folgende Maßnahmen werden unter dem ersten Punkt besonders diskutiert:

a. Spezielle Steueraufkommensgrenzen

b. Begrenzungen der Bemessungsgrundlage

c. Steuersatzbeschränkungen

\section{a. Spezielle Steueraufkommensgrenzen}

Unter diesen Regeln sind Grenzen gemeint, die die Aufkommen aus speziellen Einzelsteuern ihrer Höhe nach begrenzen. Die Art der Grenze kann dabei verschiedene Formen annehmen; in der Regel handelt es sich um solche, bei denen die Aufkommen auf einen bestimmten Anteil am Pro-Kopf-Einkommen oder auf den Zuwachs des Pro-Kopf-Einkommens bezogen sind. Im wesentlichen werden dadurch bestimmte Einzelsteuerquoten festgeschrieben, ohne daB allein durch diese Begrenzung schon eine Limitierung der a)ternativen Handlungsparameter gegeben wäre.

In diesem Punkt ist auch der Nachteil dieser Regelung aus dem verfassungsmäBigen Betrachtungswinkel zu sehen: Die Regelung ist bereits so speziell, daB die Identifikation von Gewinnern und Verlierern solcher Maßnahmen relativ einfach sein dürte. Die Entscheidung zugunsten solcher Regelungen ist somit schon zu interessengebunden und damit 
eine Entscheidung, die nach der BUCHANAN/TULLOCK-Klassifikation auf die operationale Ebene gehört - eine Kritik, die auch auf die ubrigen speziellen Regelungen zutrifft.

Zudem ist zu fragen, inwiefern eine solche Regelung uberhaupt den angestrebten $Z$ ielen gerecht werden kann. Immerhin sagt die Begrenzung des Aufkommens einer speziellen Steuer nichts über die möglichen Variationen aller anderen Steuern aus. Eine "echte" Einschränkung des politischen Handlungsspielraums durfte dadurch kaum gegeben sein. Eine tatsächliche Einschränkung ergibt sich erst dann, wenn die Aufkommensbeschränkungen alle Steuern mit hohem (potentiellen) Aufkommen betreffen und ein Steuerfindungsrecht des States nicht oder nur im begrenzten Rahmen besteht, so $d a B$ im politischen ProzeB nur geringe Spielräume zur Ausdehnung der Einnahmen bleiben. In diesem Fall wird ein ähnlicher Effekt erreicht wie durch eine allgemeine Steueraufkommensbegrenzung, allerdings mit dem Unterschied, daB die Steuerstruktur ebenfalls festgelegt ist. Auch hier ist dadurch eine Vermischung von konstitutioneller und operationaler Entscheidungsebene zu beobachten.

\section{b. Begrenzungen der Bemessungsgrundlage}

Die Begrenzung der Besteuerung auf bestimmte, möglichst eng definierte Bemessungsgrundlagen wird vor allem von BUCHANAN/BRENNAN (1980) vertreten. Ihnen schwebt im wesentlichen eine Definition eines Systems von Bemessungsgrundlagen vor, deren Umfang nicht erweitert werden kann. Das maximale Steueraufkommen ergibt sich dann als endogene Größe, da die Wahl der Steuersätze immer noch in den Händen der Politiker liegt. 


\section{c. Steuersatzbegrenzungen}

Steuersatzbegrenzungen sind die Form fiskalischer Einschränkung, die sich in der Praxis am meisten durchgesetzt hat. 1)

Allerdings setzen solche Höchstsätze, um als fiskalische Begrenzung wirksam zu werden, ebenfalls die genaue Definition und Festlegung möglicher Bemessungsgrundlagen voraus, da sonst eine beliebige Aufkommenshöhe durch eine Erweiterung der Bemessungsgrundlage erreicht werden kann bzw. die postulierte Höhe des Steuersatzes dadurch umgangen wird, indem derselbe Gegenstand mehrmals durch Bemessungsgrundlagen unterschiedlicher Definition erfaBt wird.

Auch Steuersatzbegrenzungen können auf einzelne Steuern festgelegt sein oder die Gesamtmenge der vorhandenen Steuern umfassen. Im zweiten Fall kommt dies einer Festlegung des Steueraufkommens gleich, das sich aber (nach Entwicklung der Bemessungsgrundlage) als endogene, erst ex-post feststellbare Größe herausstellt, während die Parameter des staatlichen Handelns bereits festgelegt sind. Die Beschränkung ist allerdings strenger als im Fall der noch zu behandelnden globalen Steuerbegrenzung, da durch ein System der Steuersatzbegrenzungen die Steuerstruktur ebenfalls festgelegt ist, und damit jeglicher steuerpolitischer Spielraum fehlt.

Soweit Steuersatzbegrenzungen nur einzelne Steuern betreffen, können sie nicht als angemessene Maßnahme zur Begrenzung der Staatstätigkeit angesehen werden. Hier gilt das bereits zu den Problemen der Begrenzung des Steueraufkommens Gesagte. Spezielle Steuersatzbegrenzungen

i) $\mathrm{vgl}$ dazu den Inhalt der Proposition 13 in Californien und die Regelungen in anderen amerikanischen Staaten. 
dijrften auch mehr ein Reflex bestimmter Gruppeninteressen sein, eine niedrigere eigene Steuerbelastung durchzusetzen, als mit dem expliziten Zie] einer Einschränkung des budgetären Handlungsspielraums begründet werden. Auch hier handelt es sich um "operationale" Entscheidungen mit bestimmten, abschätzbaren Begünstigungen, nicht um Maßnahmen, die die Rationalität kollektiven Handelns als solche im Sinn haben.

Fazit: Soweit spezielle Steuerbegrenzungsmaßnahmen angesprochen werden, dijfte eine Erhöhung einer Effizienz staticher Maßnahmen nicht explizites Ziel sein und auch als Beiprodukt kaum durch solche Restriktionen zu erreichen sein. Die speziellen Steuerbegrenzungsmaßnahmen, auch wenn sie die weitestverbreitete tatsächlich durchgeführte Maßnahme sein dirfte, stellt wohl eher ein Produkt des Versuchs bestimmter Bürger dar, ihre Steuerbelastung zu verringern.

\section{B. Globale Budgetgrenzen}

Globale Budgetbegrenzungen stellen eher auf eine Begrenzung der Staatstätigkeit als ganze ab als die angesprochenen speziellen Regelungen, auch wenn eine Ausdehnung von speziellen Regelungen zu einem wirklich umfassenden System zugleich zu einer allgemeinen, globalen Grenze führen wïrde. Als globale Regelungen sollen hier im einzelnen behandelt werden:
a. Eine allgemeine Verschuldungsgrenze1)
b. Eine allgemeine Aufkommensgrenze (Besteuerungsgrenze)
c. Eine allgemeine Ausgabengrenze

1) Dazuzählt auch die Forderung nach einem materiellen Haushaltsausgleich, wodurch die (mögliche) Verschuldung auf Null reduziert würde. 
Kennzeichnend für diese globalen Maßnahmen ist es, daß eine Obergrenze für ein Aggregat (Ausgaben, Einnahmen) festgelegt wird, während innerhalb dieser obergrenze der Handlungsspielraum des States weiterhin unbegrenzt bleibt. Eine Festlegung von Ausgaben- oder Einnahmenobergrenzen bedeutet $n i c h t, d a \beta$ diese auch unbedingt erreicht werden muB, ist also nicht gleichzusetzen mit einer Festlegung des Statsanteils. Eine Unterschreitung ist vielleicht nicht realistisch, aber immer möglich.

Wichtiger ist, $d a \beta$ alle Parameter innerhalb dieses Rahmens der Obergrenze frei wählbar sind. Weder die Steuerstruktur noch die einzelnen Parameter der Besteuerung sind festgeschrieben. Innerhalb der obergrenze ist jede beliebige Kombination der einzelnen Komponenten (Steuern, Ausgaben) möglich, Umstrukturierungen sind immer denkbar. Somit ist für unterschiedliche politische Prioritätensetzung noch genügend Spielraum vorhanden.

Damit soll der Unterscheidung zwischen konstitutionellen und operationalen Entscheidungen Rechnung getragen werden. Die Budgetbegrenzungen sollen den Handlungsspielraum verengen, nicht aber einen völligen Wegfall jeder Entscheidungsmöglichkeit hervorrufen.

Die drei angesprochenen Begrenzungsmaßnahmen sind nicht unabhängig voneinander: Sind $z$ wei dieser möglichen Begrenzungen vorgegeben, dann gilt auch eine Begrenzung für die dritte Kategorie. Die wichtigste der Begrenzungsarten ist dabei die Festlegung einer allgemeinen Ausgabengrenze und die (isolierte) Forderung nach einem ausgeglichen Budget, das heiBt nach materiellem Haushaltsaus gleich. Eine Besteuerungsgrenze wird in der Regel nicht ohne eine gleichzeitige Begrenzung der Verschuldungsmöglichkeiten gefordert und kommt somit implizit einer 
Ausgabenbegrenzung gleich, oder kommt für Gebietskörperschaften in Frage, die sowieso einen stark eingeschränkten Verschuldungsspielraum haben.

\section{a. Begrenzung der Schuldaufnahme}

Sehr häufig gefordert wird eine verfassungsmäBige Begrenzung der statichen Verschuldung. Diese Forderung knüpt an eine lange Tradition an, die im materiellen Haushaltsausgleich ein $z i e l$ an sich sah oder zumindest ein unverzichtbares Mittel, um andere $Z$ iele zu erreichen. Staatsverschuldung wurde allgemein nicht als "normale" Finanzierungsweise akzeptiert. Dabei stellt die Forderung nach einer verfassungsmäBigen festlegung auf einen materielien Haushaltsausgleich nur die Extremform denkbarer Beschränkung des Verschuldungsspielraumes dar, da dort die Höchstgrenze der Verschuldung auf Null festgelegt wiurde. Andere Möglichkeiten, wie zum Beispiel die Begrenzung auf eine bestimmte Verschuldungsquote als Anteil am Sozialprodukt oder die Bindung der Verschuldung an andere Größen, wie es ja im deutschen Grundgesetz für den konjunkturellen Normalfall mit der Bindung an die staatlichen Investitionen vorgesehen ist, leisten denselben Dienst. Auf die Problematik der BezugsgröBe wird später noch eingegangen.

Eine Rückführung der staatlichen Verschuldung wird in der augenblicklichen Diskussion in der Bundesrepublik ja beinahe unisono befiurwortet. Eine Begrenzung der Schuldaufnahme kann als die Begrenzung einer spezifischen Aufkommensart angesehen werden, da die Einnahmen insgesamt ja keiner Beschränkung unterworfen werden und eine Erhöhung der Ausgaben immer möglich ist, soweit damit verbunden eine Erhöhung der Steuereinnahmen erreicht wird. 


\section{b. Eine allgemeine Steuergrenze}

Als globale Maßnahme spielt die Festlegung einer maximalen Steuerquote keine so wichtige Rolle, in praxi wird eine Begrenzung der Steuereinnahmen auch nie ohne bestimmte, einschränkende Verschuldungsregeln gefordert, was somit auf eine Ausgabengrenze hinausläuft. Durch eine kombinierte Verschuldungs - und Besteuerungsgrenze ist allerdings im Gegensatz zur alleinigen Ausgabenbegrenzung eine Variation der Finanzierungsstruktur $z$ wischen diesen beiden Einnahmearten nicht mehr möglich (wenigstens, sobald eine der beiden Obergrenzen erreicht ist).

\section{c. Eine allgemeine Ausgabengrenze}

Eine globale Begrenzung der Staatsausgaben ist die von Wissenschaftlern am weitesten debattierte Form einer globalen Begrenzung. Ausgangspunkt.ist die Oberlegung, da $B$ die Menge der Ressourcen, die der Stat für sich beansprucht, durch die Höhe der staatlichen Ausgaben und nicht durch die Höhe der Einnahmen bestimmt wird. Eine Reduktion des Statsanteils durch eine Ausgabengrenze erscheint daher nur folgerichtig. Die Struktur von Ausgaben und Einnahmen bleibt weiterhin eine politisch zu bestimmende Größe, die Gesamtmenge der dem Stat zur Verfügung stehenden Ressourcen ist aber quantitativ nach oben beschränkt.

Eine allgemeine Ausgabengrenze erfiilt aber noch am ehesten die Bedingungen, die an eine verfassungsmäBige Beschränkung des Budgets zu stellen sind: Die Staatsquote ist auf einen Höchstwert festgelegt, die Struktur der Einnahmen und Ausgaben kann jedoch weiterhin variiert werden, so daB innerhalb des Rahmens ein Maximum an Handlungsspielraum bestehen bleibt. Für mögliche Varia- 
tionen der Inzidenz des Budgets bleibt somit noch genügend Platz, so daB eine Vorausschätzung, wer Gewinner oder Verlierer einer Beschränkung der Staatsausgaben ist, nicht getroffen werden kann. Eine möglichst große UngewiBtheit über die eigene Stellung, die man nach den Entscheidungen unter dem jeweiligen Verfassungsregime einnimmt, wird ja als Bedingung für eine faire Entscheidung über die Verfassungsregel genannt.

\subsubsection{Die Wahl der Basis}

Im Prinzip sind drei Arten einer Festschreibung von Quoten möglich:

1. Eine auf aktuelle Größen bezogene Quote

2. Eine auf potentielle Größen bezogeñe Quote

3. Eine objektbezogene Quote.

Zu 1.: Am häufigsten diskutiert sind die Regelungen, die eine realisierte GröBe als Bezugsbasis der Steuer-/Ausgabengrenze haben. Solche GröBen sind zum Beispiel die Höhe des Sozialprodukts oder das Pro-Kopf-Einkommen. So bezieht sich ein GroBteil der Diskussion der Festlegung einer Ausgabengrenze auf einen fixen Anteil der Staatsausgaben am Bruttosozialprodukt, wobei dies meist dadurch erreicht wird, daß der Zuwachs der Staatsausgaben den $Z$ uwachs des Bruttosozialprodukts nicht überschreiten darf und damit die Quote des Ausgangszeitpunktes als Höchstgrenze festgelegt ist.

Diese Form findet sich in den meisten der US-Bundesstaaten, die eine verfassungsmäßige Ausgabengrenze eingeführt haben.1)

i) vgl.: DWORAK (1980), WILDAVSKY (1980) 
Ein Problem bei der Beschränkung auf Zuwachsraten tritt allerdings dann auf, wenn in einem Jahr eine Zuwachsrate der staatsausgaben realisiert wird, die kleiner ist als die festgelegte maximale Zuwachsrate, nämlich, die des Sozialprodukts. Es stellt sich dann das Problem, ob nun die niedrigere Statsquote Ausgangspunkt für die Begrenzung sein soll oder ob die alte Quote beibehalten werden kann, das heiBt in den folgenden perioden ein über der maximalen Rate liegender Zuwachs möglich ist, bis die alte Quote wieder erreicht ist. Die Regelung in den US-Bundesstaten ist unterschiedlich, im allgemeinen dirfte jedoch der zweiten Regel der Vorzug zu geben sein, da dadurch ein Ausgabenzwang für die Regierung vermieden wiurde, die ihren zukünftigen Handlungsspielraum nicht eingrenzen möchte.

Eine andere Art von Bezugsgrößen können potentielle Größen darstellen, wie beispielsweise die Höhe des bei Vollauslastung des Produktionspotentials möglichen Sozialprodukts. Eine Festschreibung der Statsquote als Anteil am potentiellen Sozialprodukt hätte den Effekt, daB die Staatsausgaben von kurzfristigen Schwankungen des Auslastungsgrades relativ unberührt blieben.1) Berechnungen von GröBen auf einer solchen Basis werden zum Beispiel durch den Sachverständigenrat (allerdings unter anderen Gesichtspunkten) betrieben. Die Idee einer Festschreibung der Staatsausgaben an einer potentiellen GröBe vorzunehmen, findet sich in der Literatur selten, ist aber genauso denkbar wie eine Beziehung der Statstätigkeit auf aktuelle EinkommensgröBen.

\footnotetext{
1) Alternativ könnte man die Statsquote direkt auf das Produktionspotential beziehen, das allerdings keine "potentielle Größe" darstellt.
} 
Als letzte Möglichkeit findet sich noch die Idee, als Bezugsgröße eine bestimmte ObjektgröBe zu wählen. Diskutiert wird dies meist bei einer Bindung der Staatsverschuldung an die statlichen Investitionen als obergrenze. Auch hier wird durch die Begrenzung auf eine bestimmte BezugsgröBe ein verbindicher Rahmen für die maximale Ausdehnung des Budgets beziehungsweise bestimmter Budgetposten vorgegeben.

Welche BezugsgröBe auch immer gewählt wird, wichtig ist, daB die Berechnung dieser Größe eindeutig geregelt ist und Manipulationen durch die Instanz, die auch das Budget aufstellt, nicht zugänglich ist. Bei allen genannten Größen ergibt sich aber, daB keine davon einfach empirisch zu konstatieren ist, sondern alle erst durch Berechnung gewonnen werden können. Dabei spielt die Methodik der Berechnung für das Ergebnis eine entscheidende Rolle, wobei die Manipulationsmöglichkeiten mit dem Anteil der Schätzungen und der Unklarheit der definitorischen Abgrenzung steigen. Damit stellt sich die Notwendigkeit, entweder eine eindeutige Regel für die Berechnung der BezugsgröBe konstitutionell zu fixieren, wobei die einzelnen Berechnungselemente dann politischen Manipulationsmöglichkeiten unzugänglich bleiben, oder eine Instanz zu bestimmen, die die jeweiligen Berechnungen vornimmt beziehungsweise die Berechnungsweise bestimmt und deren UnbeeinfluBbarkeit ebenfalls zu gewährleisten ist. Beides diufte in praxi wohl einige Probleme aufwerfen.1) Festzuhalten ist auf jeden Fall, daB die konkrete Ausgestaltung der Bezugsgröße, solange sie nur eindeutig definiert ist, nicht die entscheidende Rolle spielt: Es geht ja nicht um die Bestimmung des optimalen Staatsanteils, sondern um die Veränderung der Handlungsweise der am politischen ProzeB Beteiligten, die durch eine solche Begrenzung erreicht

i) Vglo dazu in Bezug auf eine staatsverschuldungsgrenze STERN (1983) 
werden soll. Egal, welche BezugsgröBe auch immer gewählt wird, durch die Festschreibung einer Quote ist die Höhe der Staatsausgaben (Schuldaufnahme, Steuereinnahmen) dem Entscheidungsspielraum der politisch Tätigen entzogen.

Im folgenden wird die Diskussion im wesentlichen auf die Wirkungen einer allgemeinen Ausgabengrenze beschränkt sein. Eine Einschränkung der Verschuldungsmöglichkeiten wird hier deswegen nicht behandelt, weil bereits einschlägige Arbeiten zu diesem Komplex vorliegenl), auf die verwiesen werden kann. Eine allgemeine Steueraufkommensgrenze spielt für sich genommen in der Diskussion keine Rolle: Wenn diese Möglichkeit angesprochen wird, dann wird meist die Forderung nach einer gleichzeitigen Verschuldungsgrenze erhoben, so daB die Einführung einer Steueraufkommensgrenze auf eine Ausgabenbeschränkung hinausläuft, oder die Begrenzung der Steuern wird mit dem expliziten $Z$ iel verfolgt, dadurch eine Reduktion der öffentlichen Ausgaben herbeizuführen.

Spezielle Steuerbegrenzungen $z u$ behandeln, verbietet sich deshab, da hier die Verteilungswirkungen im Vordergrund der Diskussion stehen mussen und damit die Ebene der konstitutionellen Entscheidung verlassen wird. Wenn also im folgenden von Budgetbegrenzungen die Rede ist, so ist damit eine allgemeine Ausgabengrenze gemeint, soweit nicht explizit andere Beschränkungen benannt werden.

1) $v$ gl. BAUM (1982) 
3. Politische Entscheidungsmechanismen und Budgetgrenzen

3.1 Das Grundmodell des staatichen Bereitstellungsprozesses

Um eine Analyse eines möglichen Staatsversagens zu liefern, muß zumindest in Grundzügen eine Vorstellung über de Art, wie statiliche Entscheidungen zustande kommen, entwickelt werden. Hier bietet sich die Theorie der "public choice" an, die gerade die Eigenarten des politischen Entscheidungsprozesses in den Vordergrund der Analyse stellt.

Für die hier angesprochene Problematik erweist es sich als zweckmäßig, im Rahmen des Entscheidungsprozesses uiber die Bereitstellung öffentlicher Leistungen und bei der Bereitstellung selbst drei Pole zu unterscheiden, denen jeweils eine spezielle Funktion zukommt. Folgende Abbildung soll die Interaktion dieser drei Pole verdeutlichen.

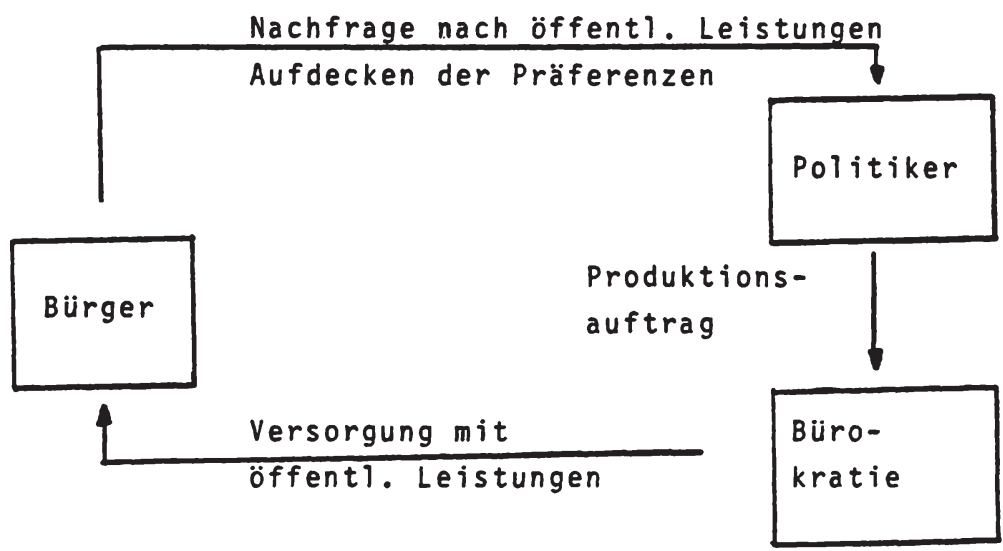


Auf der einen Seite stehen die Privaten (Bürger). Diese fragen die öffentlichen Leistungen nach, bezahlen sie aber auch durch Steuern bzw. durch die Zeichnung von Anleihen. Auf der anderen Seite steht die Administration (Verwaltung, Biirokratie) als Anbieter der öffentlichen Leistungen. Sie sorgt für die Bereitstellung öffentlicher Güter bzw. organisiert die Umverteilung von privaten Guitern (Einkommen) unter den Bürgern; die dafür nötigen Mittel werden ihr zugewiesen. Zwischen Bürger und Burokratie treten (quasi als Auktionator) die Politiker: Sie nehmen (im Idealfall) die Präferenzen der Bürger über die Versorgung mit statichen Leistungen auf und geben sie an die Bürokraten weiter.

Es muB darauf hingewiesen werden, $d a B$ die Trennung in die drei Pole "Bürger", "Politiker" und "Buirokratie" rein funktional zu verstehen ist. Eine personelle Identität ist damit durchaus vereinbar: Beamte sind sowohl Mitglieder der Administration als auch Wähler (Bürger); auch Politiker uben das Wahlrecht aus, zahlen Steuern und nehmen Statsleistungen in Anspruch.

Das Eigentümliche des Prozesses kollektiver Entscheidung ist, daB kein direkter Tausch stattfindet. Am Markt stehen sich ebenfalls Nachfrager und Anbieter als zwei Pole gegenüber, die aber jeweils autonom entscheiden und zu einer Teilnahme am Interaktions-/TauschprozeB nicht gezwungen sind. Dies trifft bei statichen Entscheidungen nicht mehr zu. Die Bürger können sich nur bedingt aus dem ProzeB der öffentlichen Produktion ausschalten - Steuern sind Zwangsabgaben, keine freiwilligen Leistungen. Ihre Erhebung bedarf nicht der Zustimmung des jeweils betroffenen Steuerzahlers. Auch vom Konsum öffentlicher Güter können sich die Individuen nicht immer ausschließen. 
Ein ideales System der kollektiven Entscheidung würde dafür sorgen, daß die Politiker die Präferenzen der Bürger richtig in Erfahrung bringen und entsprechende Produktionsaufträge an die Administration erteilen. Weiterhin müBte die staatliche Bürokratie diese Produktionsaufträge ohne Einschränkung übernehmen und die Bereitstellung öffentlicher Leistungen danach ausrichten. Ein solches System könnte dann ohne Ineffizienzen funktionieren.

In diesem einfachen Grundschema können aber auch die Ansatzpunkte gezeigt werden, die zu einem wie oben beschriebenen "Statsversagen" führen.

\section{A. Die Beziehung zwischen Bürger und Politiker}

Notwendige Voraussetzung für eine korrekte Umsetzung in Produktionsaufträge ist, daß den Politikern die Präferenzen der Bürger auch richtig vermittelt werden. Es ist dabei nicht unbedingt notwendig, daß die Präferenzen selbst den Politikern offenbart werden, sondern es genügt ein Indikator, der diese widerspiegelt, wie es beispielsweise die Preise im MarktprozeB sein sollen. Richtige Entscheidungen können nur auf richtigen Informationen basieren; gibt es keinen Mechanismus, der diese Informationen vermittelt, so ist die Effizienz der Ergebnisse der Entscheidungsfindung reiner Zufall.

Aber selbst wenn die richtigen Informationen vorhanden sind, ist noch nicht gewährleistet, daB die Politiker ihre Entscheidungen auch daran ausrichten. Dazu muB alternativ eine von beiden Bedingungen erfüllt sein:

1. Die Politiker haben keine von denen der Bürger abwei-

chenden Präferenzen. Ziel der Politiker ist es, die Wünsche der Bürger möglichst korrekt zu erfüllen. Richtige 
Informationen vorausgesetzt, erweisen sich die Politiker damit als reine Erfüllungsgehilfen der Bürgerwinsche. Vorstellungen dieser Art sind es, die der Fiktion des "wohlmeinenden Diktators" der herkömmlichen Wohlfahrtstheorie zugrunde liegen.

2. Es existiert ein wirksamer Sanktionsmechanismus, der ein von den Präferenzen der Bürger abweichendes Verhalten der Politiker hinreichend hart bestraft. Selbst wenn die Politiker andere $Z$ iele verfolgen als die Bürger, können sie diese nur dann durchsetzen, wenn keine Kontroll- und Sanktionsmöglichkeiten seitens der Bürger vorhanden sind bzw. wenn diese nicht ausgenutzt werden. Eine notwendige Voraussetzung für ein vom Bürgerwillen abweichendes Verhalten der Politiker ist somit, $d a B$ iberhaupt ein diskretionärer Spielraum vorhanden ist, im Rahmen dessen die Politiker ihren eigenen Interessen nachgehen können. Erst dann kann es zu einer Verfälschung des Bürgerwillens im politischen ProzeB kommen. Dabei ist jede dieser beiden Bedingungen hinreichend, um eine optimale Anpassung des Handelns der Politiker an die Präferenzen der Bürger zu garantieren.

Sollte ein Statsversagen dadurch begriundet sein, daB in der Beziehung Bürger - Politiker eine optimale Abstimmung nicht zustande kommt, so soll das als "Staatsversagen auf der Nachfrageseite" bezeichnet werden: Die geäuBerte Nachfrage nach öffentlichen Leistungen, d.h. die Produktionsaufträge, die die Politiker an die Administration erteilen, stimmen nicht mit den tatsächlichen Präferenzen der Bürger überein. 
B. Die Beziehung zwischen Politiker und Administration

Eine weitere mögliche Fehlerquelle besteht bei der Umsetzung der Produktionsaufträge der Politiker in die tatsächliche Leistungserstellung durch den statilichen Produktionssektor, die Administration oder Burrokratie. Auch hier sind es dieselben Punkte, wie sie schon als maBgebend für ein Funktionieren der Beziehung Bürger -Politiker genannt wurden.

Einmal müssen die Produktionsaufträge die richtigen Informationen enthalten, d.h. die tatsächliche Nachfrage an die Bürokratie weitergeben. Dies wird im folgenden als gegeben angesehen. Die Verwaltung ist ja an die gesetzlichen oder außergesetzlichen Weisungen ihrer politischen Auftraggeber gebunden, prinzipielle Verständnisschwierigkeiten uber diese Anweisungen dürften auf der Seite der Bürokratie wohl kaum vorkommen. Damit es unter dieser Voraussetzung zu einem ineffizienten Angebot an öffentlichen Leistungen kommt, muB analog zur Nachfrageseite jede der beiden folgenden Bedingungen erfüllt sein:

1. Die Buirokratie hat kein Eigeninteresse an einer optimalen Abstimmung des Angebots an öffentlichen Leistungen auf die von den Politikern geäuBerte Nachfrage bzw. verfolgt eigene $z$ iele.

2. Es gibt keinen wirkungsvollen Sanktionsmechanismus gegen ein mögliches Fehlverhalten der öffentlichen Verwaltung - die Bürokratie kann ihre eigenen Interessen gegen die Interessen der Nachfrager durchsetzen. Dabei kommt es auf die faktische Wirksamkeit, nicht nur auf die rein hypothetische Existenz dieser Sanktionsmechanismen an. 
Ist auch nur eine von beiden Bedingungen nicht erfiult, so ist damit bereits gewährleistet, $d a B$ eine optimale Anpassung des Angebots an die von den Politikern geäuBerte Nachfrage stattfindet. Ein Staatsversagen, das durch die fehlerhafte Ausfuhrung der Produktionsaufträge der Politiker durch die Administration hervorgerufen wird, soll im folgenden als "Staatsversagen auf der Angebotsseite" bezeichnet werden.

\subsection{Das Angebot an staatlichen Leistungen}

Die folgende Analyse baut auf Ansätzen auf, die von einigen Autoren der ökonomischen Theorie der Politik entwickelt wurden, um das spezifische Angebotsverhalten statlicher Burrokratien zu beschreiben. Dabei soll im weiteren davon ausgegangen werden, daB die Administration die Möglichkeit hat, eigene Interessen zumindest teilweise gegen die Interessen der Auftraggeber (Politiker) durchzusetzen. Unter dieser Voraussetzung soll dann gezeigt werden, welche Charakteristika das Angebotsverhalten der Bürokratie aufweist, und welche Auswirkungen von einer Budgetbeschränkung ausgehen.

\subsubsection{Das Angebotsverhalten von Bürokratien}

Die spezifischen Eigenschaften bürokratischer Organisationen sind ein in Soziologie, Politikwissenschaften und rkonomie lange behandeltes Thema. Im hier erarbeiteten Zusammenhang interessiert nur das sogenannte "AuBenverhalten" der staatlichen Bürokratie, d.h. ihre Aktionen gegenüber den politischen Instanzen als ihren Auftragge- 
bern. Bahnbrechend für eine ökonomische Betrachtungsweise dieses Problems war dabei die Analyse NISKANENS (1971), dessen Grundgedanken hier dargestellt werden sollen.

Ausgangspunkt ist dabei, daß das Verhalten von Burokraten, analog zu der üblichen ökonomischen Betrachtungsweise, damit beschrieben werden kann, daß diese ihre eigenen Ziele verfolgen. Das Zielsystem der Burokratien ist dabei sehr komplex, beinhaltet Dinge wie Prestige, Einkommen usw.,1) ist aber auf jeden Fall nicht mit dem Zielsystem der Auftraggeber identisch. Dabei wird jedes einzelne Buro als homogenes Gebilde angesehen, d.h. Entscheidungs- und Abstimmungsprobleme innerhalb einer burokratisch organisierten Abteilung werden hier nicht betrachtet; analog zu NISKANEN soll jede Verwaltungseinheit durch einen "Chefburokraten" repräsentiert werden, der das Interesse seiner Abteilung vertritt.2)

Zwei weitere Eigentumlichkeiten der statlichen Bürokratie sind noch einzuführen:

1. Die Monopolstellung einer Bürokratie gegenüber dem politischen Auftraggeber und

2. die fehlenden Eigentumsrechte des Bürokraten an den von inm (etwaig) erwirtschafteten überschüssen.

Ad 1: Die Existenz einer Monopolstellung der statlichen Burrokratie ist einfach ein empirisches Faktum. Begründet wird dies damit, $d a \beta$ es kostengünstiger ist, nur eine

1) Eine Obersicht uber mögliche Ziele des Managements privater Unternehmen, die sich mutatis mutandis auch auf den staatlichen Sektor übertragen lassen, findet man Z.B. bei ALCHIAN (1965).

2) NISKANEN (1971) S. $36 \mathrm{ff}$.; die Namensgebung "Chefbürokrat" stammt von BLANKART (1975) 
Behörde mit einer bestimmten Aufgabe zu betrauen. Mehrfacharbeiten könnten dadurch vermieden werden, was gegenüber der Errichtung mehrerer Büros Vorteile aufweist. ober die Stichhaltigkeit dieser Begrüdung mag man streiten, es bleibt aber eine Tatsache, daB tatsächlich keine dieselben Leistungen anbietenden und miteinander in Konkurrenz stehenden Verwaltungen zu finden sind.1)

Ad 2: Im Unterschied zu privaten Unternehmen haben Bürokraten keine Möglichkeiten, im Rahmen der Mittelverwaltung erwirtschaftete Oberschüse sich privat anzueignen, besitzen Bürokratien keine Eigentumsrechte an den ihnen zur Verfügung gestellten Mitteln. Nichtverausgabte Beträge fließen zurück an die öffentlichen Auftraggeber, somit haben die Bürokraten kein direktes Interesse an einer möglichst sparsamen bzw. effizienten Verwendung des zur Verfügung gesteliten Budgets - ein Gewinnmaximierungs ziel wie im Fall der privaten Unternehmen besteht nicht.2) Damit sind in Grundzügen die Rahmenbedingungen gegeben, unter denen der Bürokrat seine Ziele verfolgt.

1) Vgl. WAGNER/WEBER (1975). Diese Aussage ist durchaus damit kompatibel, daß Z.B. auf verschiedenen Ebenen von Gebietskörperschaften mehrere Buros dieselbe Leistung anbieten. Wichtig ist, $d a B$ diese jeweils einer eigenen Nachfragefunktion gegenüberstehen, d.h. der Politiker nicht die Wahl hat zwischen mehreren, dieselbe Leistung anbietenden Buros, diese also nicht in Konkurrenz zueinander stehen.

2) Dies ist im Prinzip auch bei Managern privater Unternehmen, soweit sie keine Anteile besitzen, der Fall. Hier ergibt sich aber ein stärkerer Zusammenhang zwischen erwirtschaftetem Gewinn und eigener Bezahlung als im Fall staatlicher Bürokraten, wo Aufstiegschancen eher von Kriterien wie Dienstalter etc. bestimmt werden als durch Erfolgsmeldungen. 


\subsubsection{Das Grundmode 11 NISKANENS 1 )}

Ober die spezielle zielfunktion wurde bis jetzt noch wenig gesagt. Im folgenden sei angenommen, $d a B$ alle spezifischen Ziele des Bürokraten positiv mit der Höhe des inm zur Verfügung stehenden Budgets korrelieren. Damit kann die Entscheidungsfunktion des Bürokraten beschrieben werden a) s:

Max! $\quad U=U(Z)$

$$
\begin{aligned}
& d U / d Z>0 \\
& \text { und da } \text { gilt } Z=Z(B) \\
& \text { mit } d Z / d B>0
\end{aligned}
$$

Max!

$U=U(B)$, oder einfacher Max! B

Mit: $\quad$ B: Höhe des Budgets

$Z$ : (private) Ziele des Bürokraten

$U$ : Zielfunktion des Bürokraten

Das Budget wird dem Bürokraten durch die jeweilige zuständige politische Instanz zugewiesen. Diese hat eine bestimmte Nachfrage nach den Leistungen des Büros. Das bereitzustellende Budget stellt somit die Zahlungsbereitschaft der politischen Auftraggeber für die Leistungen der Bürokratie dar und kann als Nachfragekurve nach dem Output des Büros interpretiert werden. Es soll gelten:

$B=B(Q)$ mit $Q=$ Output des Büros; $d B / d Q>0, \frac{d^{2} B}{(d Q)^{2}}<0$

$\frac{d B}{d Q} \quad k a n n$ dabei als Nachfragepreis nach öffentlichem Output interpretiert werden.

i) Zum folgenden NISKANEN (1968) (1971) (1975); Obersichten über Theorien der staatlichen Bürokratie findet man bei ROPPEL (1979), BLANKART (1975), ORZECHOWSKI (1971), MUELLER (1979). 
Die Produktion von öffentlichen Leistungen verursacht Kosten. Die Kostenfunktion des betrachteten Buros soll bezeichnet werden als:

$$
C=C(Q) \text { mit } \begin{aligned}
& d C / d Q>0 \\
& d^{2} C / d Q^{2}>0
\end{aligned}
$$

Eine Optimallösung (aus der Sicht der Politiker) ist analog zur Grenzkosten-Preisregel des Marktes dann gegeben, wenn die marginale Zahlungsbereitschaft der Politiker den Grenzkosten der Bereitstellung der öffentlichen Leistung entspricht:

$$
d B / d Q=d C / d Q
$$

Der Burrokrat hingegen hat eine andere Zielfunktion. Er maximiert das inm zustehende Budget unter der Nebenbedingung, daß das Budget auch voll ausgeschöpft werden muß, d.h. als Kosten für die Bereitstellung von öffentlichen Leistungen anfallen muß. Das Verhalten der Bürokraten kann also beschrieben werden als:

$$
\operatorname{Max} ! L=B(Q)+\lambda(B(Q)-C(Q)\}
$$

daraus folgt: $\frac{d L}{d Q}=\frac{d B}{d Q}+\lambda \frac{d B}{d Q}-\lambda \frac{d C}{d Q}=0$

$$
\text { bzw. } \quad \frac{d B}{d Q}=\frac{\lambda}{1+\lambda} \frac{d C}{d Q}<\frac{d C}{d Q}
$$

Der Lagrange-Multiplikator $\lambda$ kann dabei folgendermaßen interpretiert werden: Differenziert man die Gleichung nach B, der Höhe des Budgets, ergibt sich:

$$
\frac{d L}{d B}=1+\lambda
$$


Solange einer Erhöhung des Budgets ein erstrebenswertes Ziel der Bürokratie darstellt, muB gelten

$\stackrel{d L}{-}>0$,

dB

so $d a B \quad \lambda>-1$. Des weiteren gilt, da negative Lösungen für $d C / d Q$ bzw. $d B / d Q$ auszuschlieBen sind, $d a B \lambda>0$. Damit folgt für die Lösung des Maximierungsproblems, daß $d B / d Q<d C / d Q$

Folgende Graphik kann diesen Zusammenhang verdeutlichen: 
70
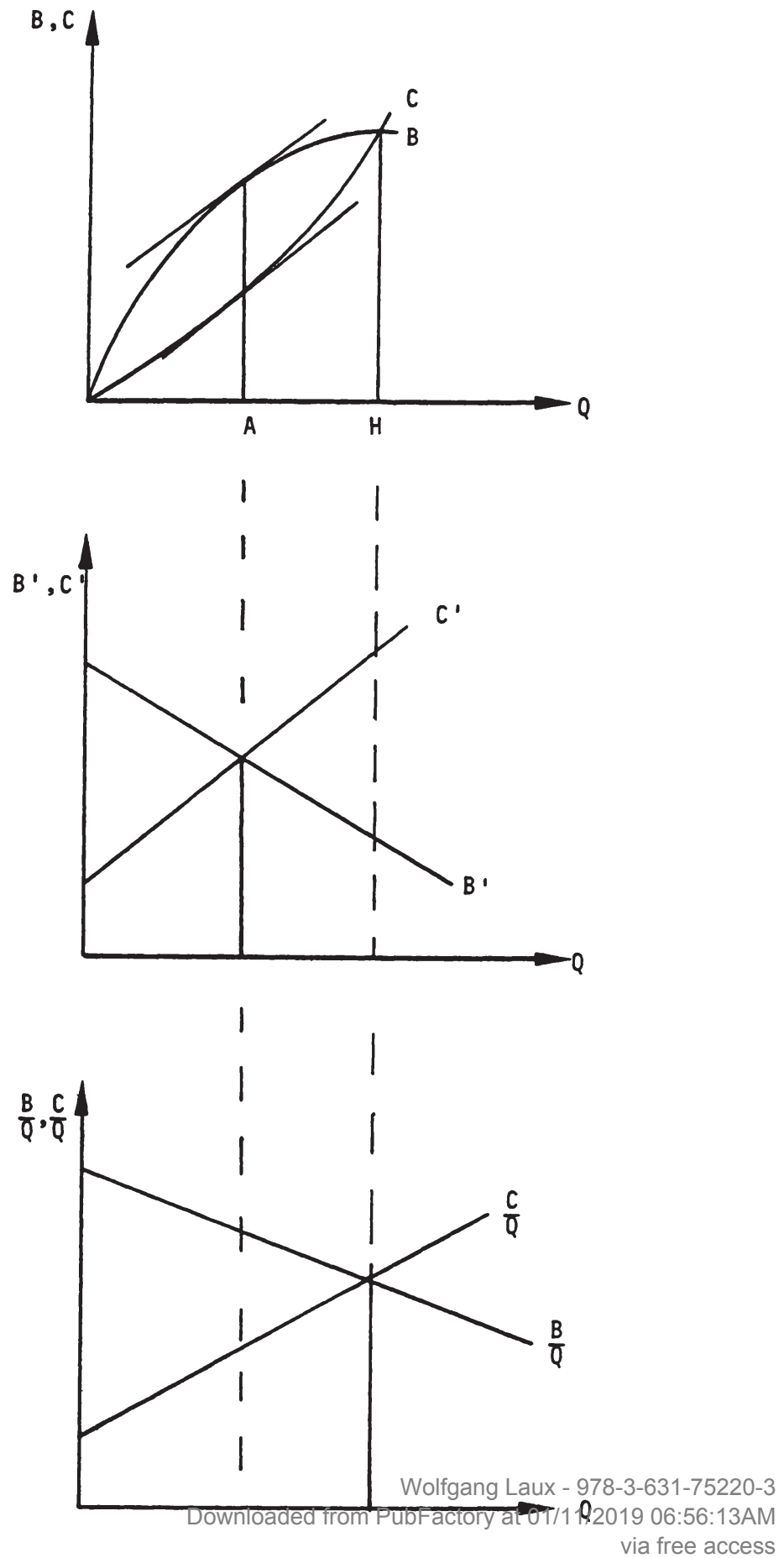
B bezeichnet dabei die Zahlungsbereitschaftskurve (oder Budgetbewilligungskurve) der politischen Auftraggeber, und kann als eine Art Indifferenzkurve zwischen öffentlichen und privaten Gütern angesehen werden. C die Minimalkostenfunktion der Erstellung der öffentlichen Leistung. Ein Optimum ist unter der üblichen Annahmen dann gegeben, wenn $B^{\prime}=C^{\prime}$ gilt, d.h., wenn die Grenzkosten der Bereitstellung der öffentlichen Leistung gleich sind der marginalen Zahlungsbereitschaft für die Leistung. Dies ist an Punkt $A$ der Fall.

Für die Bürokratie ergibt sich eine andere Sichtweise: Sie versucht, ihr Budget zu maximieren. Allerdings kann sie dies nur unter der Voraussetzung, daB die Gesamtkosten der Bereitstellung nicht die Zahlungsbereitschaft der Politiker übertreffen - dieser Fall würde zum Verzicht auf die Bereitstellung der öffentlichen Leistung überhaupt und damit zu einer Auflösung des Büros führen. Ein maximales Budget ergibt sich für das Büro dann im Punkt $H$, nämlich genau da, wo die Gesamtkosten gleich sind der gesamten Zahlungsbereitschaft der Politiker. Und genau dieses Budget wird nach NISKANEN von den Bürokraten durchgesetzt.

Zwei Bedingungen sind es, die dies ermöglichen: Ersten mussen die Bürokraten die genaue Lage der Budgetbewilligungskurve kennen. Diese an sich nicht unproblematische Annahme begriundet NISKANEN mit der langen Erfahrung der Biirokraten und der institutionelien Ausgestaltung des Budgetbewilligungsprozesses.1) Zum zweiten durfen im Gegensatz zum Büro die Politiker keine Informationen über die Lage der Minimalkostenkurve haben.

1) NISKANEN (1971) S. 28 spricht selbst davon, daB die Buros durch den ProzeB des Aushandelns zwischen Exekutive und Legislative, also verschiedenen Instanzen der politischen Auftraggeber, uiber die Nachfrage informiert sind. 
Unter diesen Umständen ist das Büro in der Lage, sich als "Optionsfixierer"1) zu verhalten: Es bietet dem Politiker eine "Alles oder Nichts"-Lösung an, d.h. stellt ihn vor die Wahl, entweder das gewiinschte Budget zu bewilligen oder ganz darauf zu verzichten. Die Politiker werden das Budget aber auch dann bewilligen, wenn nur noch eine marginale Differenz zwischen den Kosten der öffentlichen Leistung und den damit verbundenen Nutzen, wiedergegeben in der Budgetbewilligungskurve, besteht. Damit kann das Büro das gröBtmögliche Budget realisieren - die gesamte Konsumentenrente der Nachfrager wird durch das Buro abgeschöpft.2)

Das Ergebnis hat daher folgende Eigenschaften: Die bereitgestellte Menge an öffentlichem Output ist größer als die paretoeffiziente, wird aber zu Minimalkosten produziert: Das Buro produziert paretoineffizient, aber $x$-effizient. Es besteht für das jeweilige Büro sogar ein Anreiz, Ressourcen so sparsam wie möglich einzusetzen, da die Zahlungsbereitschaft mit zunehmendem output steigt, und damit das Budget für das Büro um so gröBer wird, je höher der mit den eingesetzten Mitteln erreichte Output ist. 3)

1) ROPPEL (1979) S. 106

2) Zur Möglichkeit der Beeinflussung der Nachfrage vgl. 3.3 .5 .

3) Den Fall einer mit steigender outputmenge fallenden Zahlungsbereitschaft der Politiker behandelt ROPPEL (1979) S. $110 \mathrm{ff.}$; in diesem Fall ist es möglich, daß das Budget ein Maximum erreicht hat, an dem die Gesamtkosten noch nicht die Höhe der gesamten Zahlungsbereitschaft erreicht haben. Dann besteht allerdings fuir die Bürokratie ein Anreiz, die bewilligten Mittel unproduktiv einzusetzen, da eine Erhöhung des outputs ja zur Verringerung des bewilligten Budgets führen wiurde. Dieser Fall wird aber im folgenden nicht mehr aufgegriffen, da die Voraussetzung (eine negative Bewertung des outputs) zu extrem erscheint. 


\subsubsection{Bürokratie und diskretionäres Budget}

Ausgehend von den gleichen Grunduberlegungen wie NISKANEN kommen MIGUE/BELANGER (1974) zu anderen Ergebnissen: sie akzeptieren zwar, daß die Bürokraten kein Eigentumsrecht an dem von ihnen erwirtschafteten OberschuB haben und deswegen keinen produzieren werden; allerdings ist nach ihrer Ansicht nicht die Maximierung des zur Verfïgung stehenden Budgets die Zielfunktion der Bürokratie.

Auch hier wird angenommen, $d a B$ die Büros alle ihnen zur Verfügung stehenden Mittel ausschöpfen müssen - jedoch hat die Bürokratie eine gewisse Freiheit bei der Entscheidung iber die Verwendung der Mittel: Sie kann dieseczur Produktion der öffentlichen Leistungen einsetzen, sie kann aber auch Ausgaben tätigen, die nicht zu einer erhöhten Leistungsabgabe führen, sondern den spezifischen zielen des jeweiligen Burrokraten dienen.1) Diesen Teil des Budgets bezeichnen MIGUE/BELANGER in Anlehnung an WIL LIAMSON (1964) als "diskretionäres Budget".

Grundannahme des Modells soll also sein, daß das jeweilige Buro nicht sein Gesamtbudget, sondern nur den diskretionären Teil des Budgets maximiert. Die Höhe des diskretionären Budgets ergibt sich dabei als Differenz zwischen dem bewilligten Budget und den für die Herstellung der öffentlichen Leistung benötigten Mitteln. Die Zielfunktion des Büros lautet also:
Max!
$B^{D}$
mit
$B D=B(Q)-C(Q)$

Notwendige Bedingung ist dann:

$$
d B / d Q=d C / d Q
$$

Folgende Zeichnung soll die Zusammenhänge verdeutlichen:

1) z.B. durch teure und repräsentative Buiroausstattung, Einstellung von Mitarbeitern usw. 
74

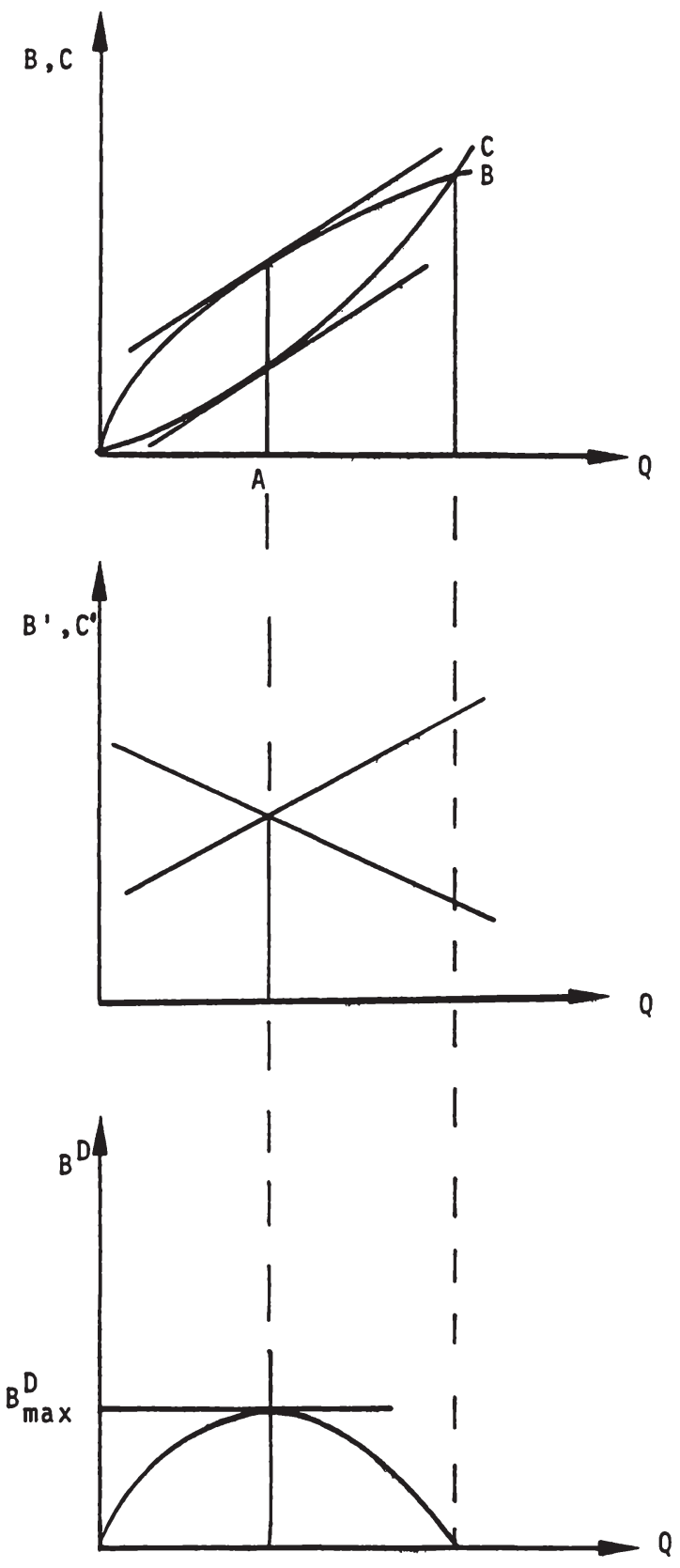

Wolfgang Laux - 978-3-631-75220-3

Downloaded from PubFactory at 01/11/2019 06:56:13AM

via free access 
Ein Maximum für das diskretionäre Budget ergibt sich dann, wenn (unter Ausschöpfung der gesamten Konsumentenrente) die Buirokratie genau die Menge an öffentlichen Gütern bereitstellt, bei der die Grenzkosten-Preisregel erfuilt ist. Die Analogie zum vollkommen preisdiskriminierenden Monopolisten ist offensichtlich.

Das Büro erstellt die paretooptimale Menge öffentlicher Giuter, aber zu über den Minimalkosten liegenden Durchschnittsausgaben.1) "Budgets of bureaus are too large but output is not necessarily so. It is seen that on theoretical grounds the problem of bureaus is not generally one of oversupply. The problem resides in bureaucrats enjoying rents at the expense of the consumer".2)

\subsubsection{Ein gemischtes Zielsystem der Bürokratie}

Der Vollständigkeit halber soll noch der Fall beschrieben werden, bei dem beide Motive: Maximierung des Budgets und Maximierung des diskretionären Budgets im Zielsystem der Bürokratie zu finden sind.3)

1) Das impliziert nicht, daB diese Situation ein Paretooptimum darstellt. Im Unterschied zum vollkommen preisdiskriminierenden Monopolisten, bei dem die Verwendung des Gewinns unabhängig ist von der Entstehung, können sich die Bürokraten das diskretionäre Budget nur dadurch aneignen, indem sie die Mittel in einer spezifischen Weise verausgaben. Es ist nicht zu erwarten, daß das Ergebnis allokativ optimal ist - die Mittel können $j a$ nicht in jeder beliebigen Art und We ise verausgabt werden, so daß den Bürokraten nur eine zweitbeste Methode zur Erhöhung des eigenen Nutzens zur Auswahl steht.

2) MIgUE/BELANGER (1974) S. 34

3) Dieses Modell ist ähnlich den von MIGUE/BELANGER (1974) und NISKANEN (1975) beschriebenen Modellen. Dort ist jedoch der Output an öffentlichen Leistungen, nicht die Höhe des Budgets, neben dem diskretionären Budget in der Nutzenfunktion vertreten. Da das Budget und der output aber positiv miteinander verknüpt sind, können die Ergebnisse ohne weiteres ibbertragen werden. 
Das Maximierungsproblem lautet dann:

$\operatorname{Max!}$

$$
L=U(B, B D)+\lambda\{B D-B(Q)+C(Q)\}
$$

Es ergibt sich als Lösung:

(1) $\frac{\partial L}{\partial B}=\frac{\partial U}{\partial B} D+\lambda=0$

(2) $\frac{\partial L}{\partial T}=\frac{\partial U}{\partial B} \frac{d B}{d Q}+\lambda\left\{\frac{d C}{d Q}-\frac{d B}{d Q}\right\}=0$

(3) $\frac{\theta L}{\partial \lambda}=B^{D}-B+C=0$

Setzt man (1) in (2) ein, ergibt sich:

$\frac{\partial U}{\partial B} \frac{d B}{d Q}=\frac{\partial U}{\partial B}\left\{\frac{d C}{d Q}-\frac{d B}{d Q}\right\}$

BZW.:

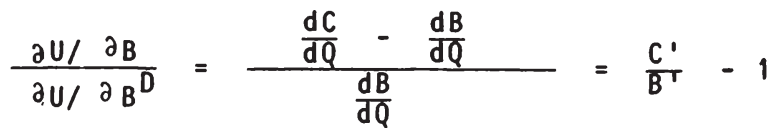

Zeichnerisch läßt sich dieser Zusammenhang folgendermaßen darstellen: 


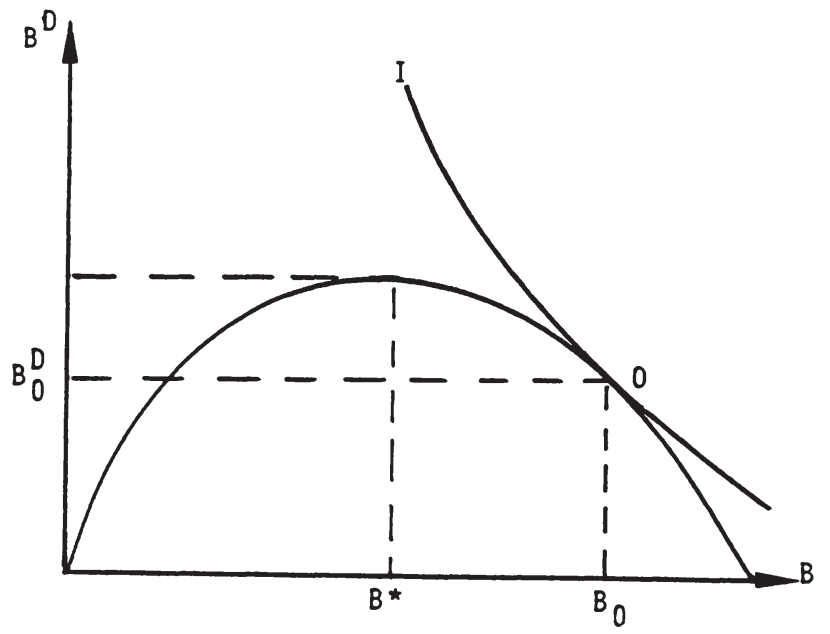

Es besteht ein trade-off $z$ wischen Höhe des Budgets und Höhe des diskretionären Budgets, sobald die optimale BudgetgröBe (aus Sicht der Gesellschaft) überschritten wird. Für die Bürokratie ergibt sich als optimaler Punkt der Tangentialpunkt zur höchstmöglichen Indifferenzkurve, die die relative Gewichtung von BudgetgröBe und diskretionärem Budget in der Zielfunktion der Bürokratie symbolisiert.

Das gewählte Budget verbindet die beiden Ineffizienzen der bereits besprochenen Fälle: Es liegt über dem paretooptimalen $B u d g e t\left(B^{*}\right)$ und weist $z$ ugleich einen hohen Grad an X-Ineffizienz auf (in Höhe von $B_{0} D$ ).

\subsubsection{Bürokratie und politische Kontrolle}

Eine der wichtigsten Voraussetzungen für die Gültigkeit der bisher abgeleiteten Ergebnisse war, daB die Politiker über keine Informationen uiber den tatsächlichen Verlauf der Kostenfunktion der Bereitstellung der öffentlichen Leistungen verfügen. 
Kennen die Politiker den Verlauf der Minimalkostenkurve, so können sie durch einfache Anweisungen ihre Vorstellungen über die Höhe und Ausgestaltung des optimalen Budgets durchsetzen.

Nur wenn den Politikern die notwendigen Informationen fehlen, gelingt es den Birrokraten, ihr abweichendes Verhalten durchzusetzen. Allerdings können Politiker sich auch Informationen beschaffen und damit eine Kontrolle uiber das Verhalten der Bürokratie ausüben.1) Die Beschaffung von Informationen ist allerdings kein kostenloser ProzeB. Entsprechend haben die Politiker die Grenzerträge einer Verminderung bürokratischer Ineffizienz gegen die Grenzkosten der Beschaffung von Informationen, die benötigt werden, um konkrete Anweisungen an das Büro zu geben, abzuwägen. Eine vollständige Kontrolle dürte daher, außer bei kostenloser Informationsbeschaffung, kaum vollzogen werden.

Die Notwendigkeit der Ausübung von Kontrolle entsteht durch die Existenz divergierender Ziele von Burokratie und deren politischer Auftraggeber - läge Zielidentität vor, so wären Kontrolle und die damit verbundenen Kosten nicht notwendig.2) Diese Kosten stellen aber für sich genommen auf jeden Fall einen Verlust für die Gesellschaft dar.

i) Vgi. BRETON/WINTROBE (1975)

2) BRETON/WINTROBE (1975) S. 201 weisen auf die Identität der Ergebnisse kostenloser Informationsbeschaffung zur Kontrolle durch die Politiker und uneigennützigen Verhaltens der Bürokratie hin. 


\subsubsection{Budgetbeschränkung und Bürokratie}

Als eine Möglichkeit, um die aus der Handlungsfreiheit von Bürokratien entstehenden Ineffizienzen abzubauen, wird eine Beschränkung des zur Verfügung stehenden Budgets genannt.1) Die Auswirkungen einer solchen Budgetbeschränkung sind nun unter den Annahmen des vorher entwickelten Modells bürokratischen Verhaltens zu untersuchen.

\subsubsection{Der NISKANEN-Ansatz}

\section{A. Ein universelles Büro}

Angenommen wird, daB dem Politiker der gesamte Sektor der statichen Bürokratie als ein einheitliches organ gegenubersteht, d.h., die gesamte Bürokratie läßt sich durch ein einziges Büro symbolisieren. Eine Einschränkung des Budgets hat dann zur Folge, daB die Zielfunktion des Büros nicht mehr in der alten Form aufrechterhalten werden kann. Ist dem Büro die Höhe des maximalen Budgets vorgegeben, dann macht eine budgetmaximierende Strategie keinen Sinn mehr. Gehen wir weiter davon aus, daB das Büro zu Minimalkosten produziert, ergibt sich folgendes Bild:

i) zum folgenden: TOMA/TOMA (1980) 


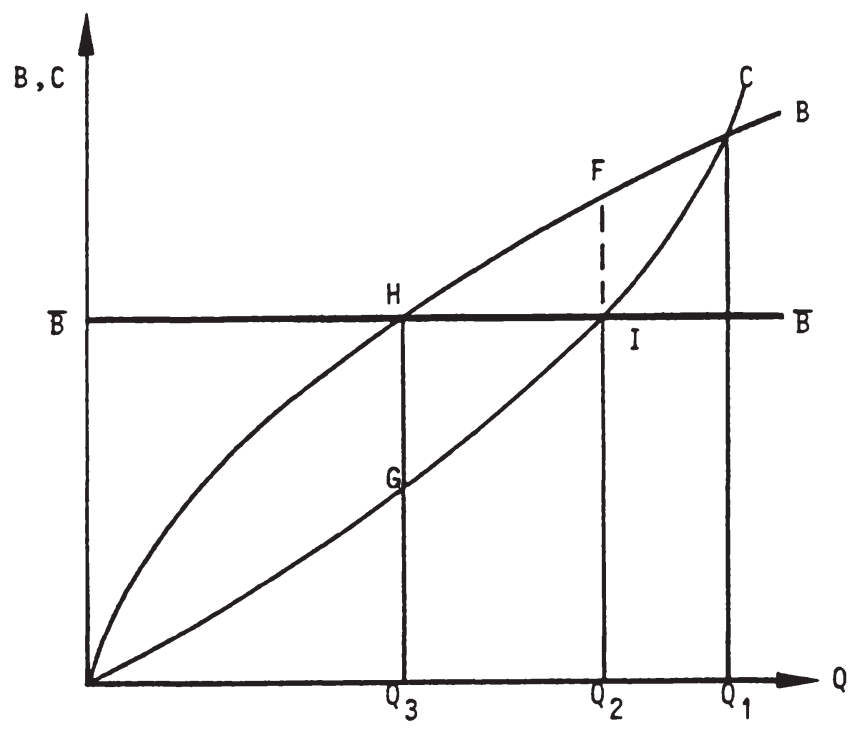

Durch die Budgetbegrenzung wird das Maximalbudget auf $\bar{B}$ festgelegt; gegenüber der Ausgangsmenge $Q_{1}$ kann das Büro höchstens die verringerte Menge $Q_{2}$ an öffentlichem Output produzieren. Gegenüber der Ausgangssituation ist damit auf jeden $\mathrm{Fall}$ eine Verbesserung für die Wähler verbunden: Sie wären bereit, die Menge $Q_{2} F$ an Mitteln zur Verfügung zu stellen, die tatsächlichen Kosten sind aber durch das (kleinere) Budget $\bar{B}$ vorgegeben. Somit entsteht ein zur Verfügung stehender OberschuB in Höhe von FI, der den in Geldeinheiten ausgedrückten Gewinn für die privaten (oder politischen) Auftraggeber des Büros darstellt. Für die Existenz eines solchen Gewinns spielt dabei keine Rolle, 
ob die Menge $Q_{2}$ über oder unter der optimalen Menge der öffentlichen Leistungen liegt: In jedem Fall ergibt sich ein Nettovorteil aus der Beschränkung des Budgets, der allerdings um so höher ist, je näher das tatsächliche Budget $B$ am optimalen Budget liegt.

Allerdings entfällt jetzt für die Burokratie jeder positive Anreiz, zu minimalen Kosten zu produzieren. Wie bereits gesehen, entstand dieser ja durch den Umstand, daB jede Abweichung von der Minimalkostenkurve zu einem verringerten Budgetumfang fuihrte und somit im Widerspruch zur Zielfunktion des Bürokraten stand. Dies ändert sich jetzt. Ist die Größe des Budgets vorgegeben, so zieht die Bürokratie keinen Vorteil aus einem kostenminimierenden Verhalten, solange die Budgetbeschränkung nicht ausgeschöpft und die Gesamtzahlungsbereitschaft fur eine bestimmte Menge öffentlicher Guter noch nicht überschritten ist. Im Extremfall wird das Buro seinen output auf $Q_{3}$ verringern, wobei "tatsächliche" Kosten in Höhe von.nun $Q_{3} G$ auftreten, Mittel in Höhe $G H$ werden verschwendet. Mithin produziert das Buro nicht mehr $X$-effizient.

Gegenuber einer Situation ohne fiskalische Grenze verschlechtert sich der Auftraggeber des Bijros aber auch in diesem Extremfall nicht. Bereits die Ausgangssituation war ja dadurch gekennzeichnet, daB die Bürokratie alle mögliche "Konsumentenrente" des Auftraggebers ausschöpfte, d.h. dieser keinen Nettogewinn (oder nur einen marginalen) realisierte. An dieser Situation ändert sich im betrachteten Fall nichts. Insgesamt kann in diesem Fall eine Budgetbeschränkung für den politischen Auftraggeber nur einen Gewinn erbringen, der im ungünstigsten Fall gleich Null wird. 


\section{B. Mehrere Büros}

Die Annahme eines einzigen Büros soll jetzt fallengelassen und statt dessen davon ausgegangen werden, $d a B$ mehrere Büros existieren. Das Gesamtbudget sei vorgegeben, die konkrete Aufteilung auf die verschiedenen Büros aber noch ein $z$ verhandelnder Gegenstand. In diesem Fall ist nämlich die Zuteilung von Mitteln an die einzelnen Büros nicht mehr unabhängig von den von den Büros angebotenen Leistungen, diese haben also einen EinfluB auf die Höhe ihrer Budgets.

Dazu ist allerdings erst die Reaktion der Nachfrager auf verschiedene Budgetanträge zu spezifizieren. Im folgenden wird angenommen, $d a B$ immer zuerst der Antrag berücksichtigt wird, der den höchsten oberschuB (B-C)/C für die Auftraggeber ergibt.1) Weiterhin sei angenommen, daB die Büros zwar die "Nachfragefunktion" der politischen Auftraggeber und die Kostenfunktion für ihren eigenen Output kennen, nicht jedoch die Nachfrage- und Kostenfunktionen der anderen Buros. Durch die Gesamtbudgetbeschränkung wird somit eine Konkurrenzsituation unter den

1) Diese Strategie muß nicht unbedingt zum besten Ergebnis führen: Es kann sogar sein, daB durch diese Art der Budgetzuteilung insgesamt eine Verschlechterung stattfindet. Ein Büro, das kostenminimal, aber in einem Bereich arbeitet, in dem die Menge der öffentlichen Leistung höher ist als die "paretoeffiziente", kann durchaus ein höheres durchschnittliches Nutzen-KostenVerhältnis ausweisen als ein Büro, das ebenfalls kostenminimal, aber unter dem paretoeffizienten Niveau arbeitet. Eine Umverteilung von diesem zum ersten Büro ist dann aber als schlechter zu beurteilen als eine Erhöhung des Budgets des zweiten auf Kosten des ersten Büros. Optimal wäre für die Nachfrager eine Orientierung an der Differenz: Grenzkosten-marginale Zahlungsbereitschaft - erstere sind dem Nachfrager aber nicht bekannt, so daß diese Strategie ausfällt. Eine Orientierung an Durchschnittswerten ist dann als Versuch der Nachfrager zu werten, die Grenzkosten irgendwie zu approximieren. 
einzelnen Büros geschaffen, die dadurch jeweils ein höheres eigenes Budget erreichen können, indem sie die Nutzendifferenz $(B-C) / C$ für die eigenen Leistungen möglichst hoch ausweisen. Für jedes Büro entsteht so bei ineffizienter, outputreduzierender Produktion die Gefahr, daB ein anderes Büro eine bessere Nutzen-Kosten-Relation aufweist, und somit Mittel aus dem eigenen Bereich abgezogen und anderen Büros zur Verfügung gestellt werden.

Ein budgetmaximierendes Büro wird bei UngewiBheit über das Verhalten anderer Büros bestrebt sein, möglichst zu Minimalkosten zu produzieren, da es sonst Gefahr läuft, vermeidbare Kürzungen zu erleiden. Diese Tendenz wird dadurch noch verstärkt, daB im NISKANEN-Modell die einzelnen Büros nur an der Höhe des Budgets interessiert sind, mithin gegenüber der Art der Verausgabung der Mittel (zur Produktion öffentlichen Outputs oder als waste) indifferent sind. 1 )

Dieses Ergebnis zeigt starke Parallelen zu der Argumentation von MCGUIRE/COINER/SPANCAKE (1979): Diese weisen nach, daB in einem Mehr-Büro-Fall unter der Voraussetzung, daB die von den einzelnen Büros produzierten outputs substitutiv sind, ein WettbewerbsprozeB zwischen den verschiedenen Büros dazu führt, daB die Auftraggeber einen positiven Oberschuß über die Kosten realisieren. Der reine NISKANEN-Fall, bei dem wie gesagt die gesamte Konsumentenrente der Auftraggeber ausgeschöpft wird, gilt weiter unter der Voraussetzung, daß die von den Büros erstellten öffentlichen Leistungen in einem komplementären Verhältnis stehen oder unabhängig voneinander sind. Der Mechanismus

1) Es besteht also auch kein Anreiz, zur gemeinschaftlichen Ausbeutung des Nachfragers ein Kartell zu bilden bzw. Absprachen $z$ wischen den verschiedenen Büros zu treffen. Die einzelnen Büros spielen ein reines Nullsummenspiel gegen die anderen, fur Koalitionen ist da kein Platz. 
entspricht im wesentlichen dem eben beschriebenen. Durch die Einführung der Budgetbeschränkung wird hier "künst lich" eine Substitutionsbeziehung zwischen den Outputs der einzelnen Büros geschaffen: Je höher der Effizienzgrad der anderen Büros ist, desto größer sind die EinbuBen im eigenen Budget und um so gröBer ist der Anreiz, durch Effizienzverbesserungen anderen Büros Mittel zu entziehen.

Als Ergebnis läBt sich festhalten: Im NISKANEN-Modell dürfte eine bindende Budgetbeschränkung zu Verbesserungen führen, zumindest eine Verschlechterung der Nachfrager ist ausgeschlossen.

\subsubsection{Die Budgetbeschränkung im MIGUE-BELANGER-Fall}

A. Ein universelles Büro

Im folgenden sollen die Auswirkungen einer Budgetbeschränkung dargestellt werden, wenn die $Z$ ielfunktion des Büros die Maximierung des diskretionären Budgets ist. Bei vorgegebenem Maximalbudget wird dann das Büro den kleinstmöglichen Output produzieren, den die Nachfrager noch zu akzeptieren bereit sind. Wie hoch dieser output ist, soll an folgender Zeichnung gezeigt werden: 


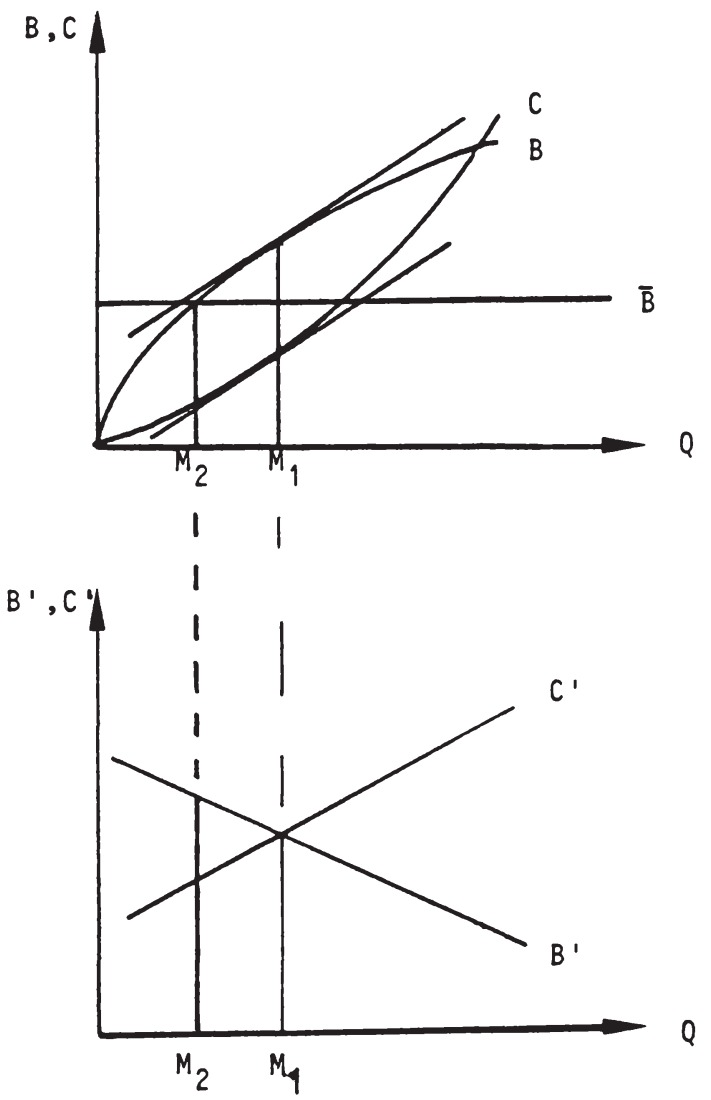

Die Strategie des Buiros besteht jetzt darin, bei gegebenem Budget die auf die Produktion der öffentlichen Leistung verwendeten Ressourcen zu minimieren, $d . h$. es wird nur das Outputniveau aufrechterhalten, das gerade ausreicht, daB die Zahlungsbereitschaft der Auftraggeber der vorgegebenen Budgetgrenze entspricht. Das ist in diesem $\mathrm{Fall}_{1}$ bei $\mathrm{M}_{2}$ der Fal1.

Die Produktion der öffentlichen Leistung wird somit auf ein Niveau sinken, daB unterhalb des paretoeffizienten Niveaus liegt. Dennoch verlieren die Nachfrager nicht. Auch im MIGUE-BELANGER-Modell schöpfen die Bürokraten die 
gesamte Konsumentenrente ab. Eine Reduzierung des outputs füht somit zu keiner Verschlechterung der Nachfrager -der Wert der weggefallenen öffentlichen Leistung entspricht genau der Verringerung des Budgets, letztendlich sind die Nachfrager indifferent gegenüber einer Änderung des Outputniveaus. Allerdings verbessern sie sich auch nicht, eine Budgetbegrenzung hilft in diesem Fall nicht weiter.1)

\section{B. Mehrere Büros}

Das Bild verändert sich, wenn man die Annahme eines einzigen Büros aufgibt. Unter den unter 2.1 .2 angegebenen Voraussetzungen kann jetzt jedes Büro seinen budgetären Spielraum erweitern, indem es die Kosten pro Outputeinheit reduziert. Ein erweiterter budgetärer Spielraum bedeutet aber auch ein erhöhtes diskretionäres Budget. Eine marginale Erhöhung des Gewinns für die politischen Auftraggeber kann einen MittelabfluB von den anderen Büros zum eigenen verursachen. Ceteris paribus kann ein einzelnes Büro seinen Anteil am Gesamtbudget und damit seinen diskretionären Spielraum dadurch ausdehnen, indem es auf die vollständige Ausschöpfung der Konsumentenrente verzichtet.

Dabei muB das Büro aber die Verringerung des inm zur Verfügung stehenden diskretionären Budgets durch eine erhöhte outputproduktion abwägen gegen die mit der erhöhten Budgetsumme verbundenen Erhöhung des diskretionären Budgets. Das jeweilige Büro sieht sich somit analog zum Monopolfall der Privatwirtschaft einer Art PreisAbsatz-Kurve gegenüber, deren Steigung und Lage aber nicht

1) Bezieht man die Veränderung der Lage der Bürokraten mit in die Beurteilung ein, handelt es sich sogar um eine Pareto-Verschlechterung. Die Bürokraten verschlechtern sich durch die Verringerung des diskretionären Budgets, während die Nachfrager keine Verbesserung ihrer Position erfahren. 
bekannt und auch über längere Frist nicht stabil sind: Das Büro wird immer nur dann einen Budgetzuschlag erhalten, wenn der den politischen Auftraggebern angebotene Gewinn aus einer Erhöhung des outputs gröBer ist als der von anderen Büros angebotene Gewinn. Somit hängt die Möglichkeit, eigene Budgeterhöhungen durchzusetzen, auch und vor allem vom Verhalten der anderen Büros ab.

Im günstigsten Fall werden die einzelnen Buros immer neue Budgetvorschläge machen und sich analog zum KonkurrenzMarktprozeB immer weiter unterbieten, bis letztendlich eine Situation zustande kommt, bei der alle Buros sich auf der Minimalkostenkurve befinden. In diesem Fall können Budgetbeschränkungen also ein sehr effizientes Mittel sein, um den diskretionären Spielraum der Büros zu minimieren und dadurch, daß die verschiedenen Büros in eine Konkurrenzbeziehung treten, Informationen ubber den "wahren" Verlauf der Kostenkurve fur die Herstellung öffentlicher Güter zu gewinnen.

Allerdings steigt (anders als im NISKANEN-Fall) hier die Wahrscheinlichkeit, $d a B$ die Büros sich zu einer Art Kartell zusammenschließen und versuchen, die für sie letztendich nachteilige Konkurrenzbeziehung zu vermeiden. Im Extremfall werden die Büros sich auf einen bestimmten Verteilungsschlussel einigen und dem politischen Auftraggeber quasi als ein einheitlicher Akteur gegenibertreten. Damit wäre man zuruick beim Fall $A$, mit der Konsequenz, daB die fiskalische Begrenzung keine Verbesserung der Situation herbeigefihrt hat.

Allerdings gilt auch in diesem Fall, daB durch die Budgetbegrenzung keine Verschlechterung eintreten kann. Zudem sind Kartelle bekanntich relativ instabil. Bei öffentlichen Verwaltungen kommt auf Grund der fehlenden Eigentumsrechte an den erwirtschafteten oberschüssen 
hinzu, daB Kompensationszahlungen von einem Büro an ein anderes nicht möglich sind, und damit ein wichtiges Instrument fehlt, ein gemeinsames Handeln herbeizufüren.

\subsubsection{Budgetbeschränkungen und gemischtes $\mathrm{Zielsystem}$}

Sind sowohl die Höhe des Budgets als auch die des diskretionären Budgets im Zielsystem der Bürokratie enthalten, so entfällt das erste $Z$ iel durch eine Budgetbeschränkung: Für den Fall, daß die Höhe des Budgets vorgegeben ist, bleibt als von der Bürokratie zu beeinflussende GröBe nur noch die Höhe des diskretionären Budgets. Dabei kann es je nach Lage der Budgetbeschränkung zu einer Erhöhung der X-Ineffizienz des Budgets kommen, ein Ergebnis, das ja aus der Betrachtung des reinen NISKANEN-Falls bekannt ist.

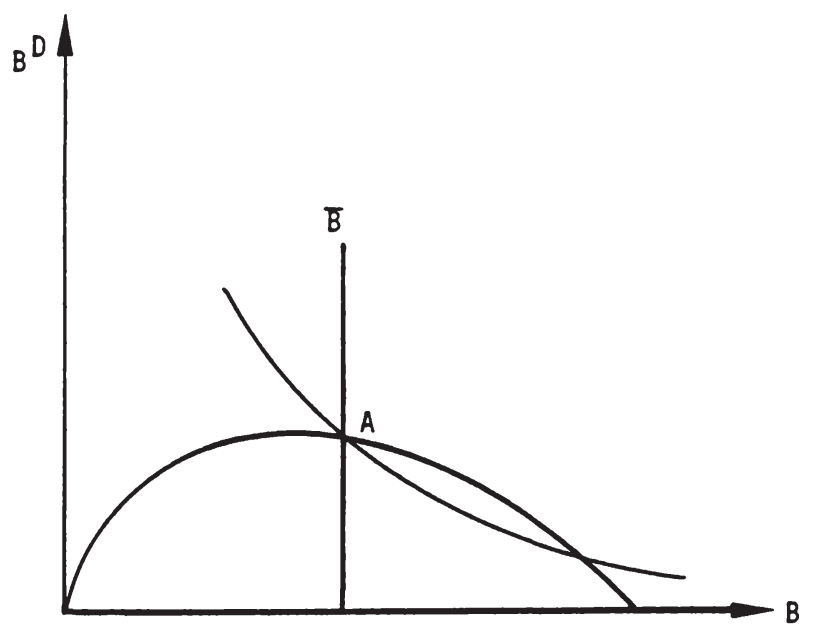


Die Zeichnung soll das verdeutlichen. Bei einer Budgetgrenze $\bar{B}$ wird das Büro Punkt $A$ realisieren, d.h. das höchstmögliche diskretionäre Budget bei gegebener Budgethöhe.

Werden mehrere Büros betrachtet, so ergibt sich keine wesentliche Änderung $z u$ den bereits diskutierten Fällen, auf eine Wiederholung der Argumente soll deshalb verzichtet werden.

\subsubsection{Budgetgrenze und Kontrollkosten}

DaB Politiker sich Informationen über die tatsächlichen Kostenverläufe bei der Produktion öffentlicher Leistungen verschaffen, war als eine mögliche Reaktion auf das eigennutzorientierte Verhalten der Bürokratie beschrieben worden. Auch hier kann die Einführung einer Budgetgrenze einen positiven EinfluB ausüben, indem die Kosten der Informationsbeschaffung, die Kontrollkosten, gesenkt werden. Zwei Faktoren sind dabei zu nennen: Erstens führt eine Budgetgrenze im Falle mehrerer Büros dazu, daB durch das Angebotsverhalten der einzelnen Büros die Identifizierung der Minimalkostenkurve für die kontrollierende Instanz einfacher, d.h. kostengünstiger wird. Zweitens ist eine Kontrolle der Bürokratie auch nicht mehr so wichtig: Der verstärkte WettbewerbsprozeB der einzelnen Buros untereinander führt zu einem ähnlichen Ergebnis, wie es eine verstärkte Kontrolle der Burokratie durch die Politiker bewirkt hätte - die Kosten der Leistungserstellung werden sinken. In diesem Fall werden auch Kontrollmaßnahmen entbehrlich. Der Wettbewerb tritt an die Stelle der Kontrolle.1)

1) vgl. MCGUIRE/COINER/SPANCAKE (1979) S. 353 


\subsubsection{Fazit}

Es zeigt sich, daB, soweit die spezifischen Angebotsbedingungen von öffentlichen Leistungen untersucht werden, die Einfuihrung von Budgetgrenzen nur positive Effekte nach sich ziehen kann. Allerdings gilt dies nur unter den angegebenen Annahmen über das Verhalten der Bürokratie, die dazu führen, daB den Statsbürgern kein Nettonutzen aus der staatlichen Leistungserstellung erwächst. Logischerweise kann dann eine Reduktion des Budgets auch keine Verschlechterung nach sich ziehen. Der wesentliche Punkt, der zu einer Verbesserung füht, ist jedoch, daB Budgetgrenzen (indirekte) Mittel sind, um eine Konkurrenzsituation zwischen der verschiedenen Abteilungen der Administration zu schaffen und somit diese zur Aufdeckung und Reduzierungen von Ineffizienzen zu veranlassen. Diese induzierte Verhaltensweise ist es, die letztendich zu einer positiven Beurteilung der Einführung von Budgetgrenzen führt.

3.3 Die Nachfrage nach Staatsleistungen im "public choice"-Paradigma

\subsubsection{Vollkommene politische Konkurrenz}

Ausgangspunkt der folgenden Analyse sind Oberlegungen, wie sie in der ökonomischen Theorie der Politik über den EntscheidungsprozeB in einer Demokratie zu finden sind und im AnschluB an die grundlegenden Werke von DOWNS (1968), 
HERDER-DORNEICH (1959) und BUCHANAN/TULLOCK (1962) formuliert wurden.1) Die Voraussetzungen sind dabei folgende: Auf der einen Seite stehen die Stimmbürger, die daruber abstimmen, welche der um ihre stimme konkurrierenden Politiker (oder Parteien) an die Regierung kommen sollen. Einziges Entscheidungskriterium ist dabei das von den jeweiligen Parteien angebotene Budget. Die Individuen werden jeweils dem Politiker ihre Stimme geben, dessen Budgetvorschlag ihnen den höchsten Nettonutzen bringt, ihren Präferenzen am ehesten entspricht.

Dabei soll Betrug ausgeschlossen sein, d.h. für die Politiker sind ihre jeweiligen Versprechungen bindend, und die Budgetvorschläge sollen auch durchsetzbar sein - die Auswahl soll somit nur zwischen tatsächlichen Zuständen möglich sein. Utopische Wahlversprechungen, die nachher nicht eingehalten werden oder werden können, sollen nicht zur Auswahl gestellt werden.

Ist diese Bedingung nicht erfült, so stellen sich groBe Probleme hinsichtlich der Kontrolle der Politiker, 2 ) die nach einer einmal gewonnenen Wahl mehr oder weniger machen können, was sie wollen. Wenn Wahlbetrug möglich ist, muß die durch die Entscheidungen der Politiker entstandene Situation nichts mehr mit den Wählerpräferenzen zu tun haben, auch wenn die Stimmbürger sich rational zwischen den angebotenen Programmen entschieden haben. Erkennen die Wähler hingegen die Möglichkeit falscher Versprechungen, dann werden sie sich nicht mehr (nur) an den angebotenen Parteiprogrammen, sondern an anderen Kriterien (Politikerpersönlichkeit usw.) orientieren. Auch das führt dazu, daß nicht mehr die von den Politikern nachträglich ergriffenen

\footnotetext{
1) Als Obersicht Z.B. MUELLER (1979), HERDER-DORNEICH/GROSER (1977)

2) vgl. BARRO (1973)
} 
Maßnahmen für die Wahlentscheidung bestimmend sind. Es läßt sich dann nicht mehr ohne weiteres ein Zusammenhang zwischen Wählerpräferenzen, Wahlentscheidung und Maßnahmen der Politiker konstruieren. Um solche Schwierigkeiten zu vermeiden, soll im folgenden davon ausgegangen werden, daß die Politiker ihre Wahlversprechen halten.1)

Auf der anderen Seite stehen die Politiker. Mit DOWNS (1968) wird angenommen, daB deren einziges $Z$ iel ist, die Wahl zu gewinnen. Ihr Verhalten soll als Stimmenmaximierung beschrieben werden können.

Als allgemeines Entscheidungskriterium gilt die Majoritätsregel, d.h. die Politiker gewinnen die Wahl, die mehr als 50\% der Stimmen auf sich vereinen können. Unter diesen Voraussetzungen ist zu untersuchen, ob die Ergebnisse eines Prozesses vollkommener politischer Konkurrenz zu einem pareto-optimalen, sprich allokationseffizienten Ergebnis führen, d.h. ob es zu einem Staatsversagen aus der Interaktion Politiker-Bürger kommen kann.

Vollkommene politische Konkurrenz2) heißt in diesem Fall auch, daß die Politiker vollkommen über die Präferenzen der Bürger informiert sind.

i) Als Begründung könnte genannt werden, daß ein Wahlbetrug bei der nächsten Wahl mit Stimmverlusten sanktioniert wird und kein Politiker seine Wiederwahl gefährden will.

2) Zum Begriff "vollkommene politische Konkurrenz" vgl. B.S. FREY (1970) 
Des weiteren sollen die Handlungsparameter der Politiker keinen A-priori-Restriktionen unterliegen, d.h. alle denkbaren Parameter (Steuersätze, Bemessungsgrundlagen usw.) sollen frei wählbar sein. Vorausgesetzt ist nur die Entscheidungsregel selbst.1)

Unter diesen Voraussetzungen ist, wie bei DOWNS bereits ansatzweise 2 ) beschrieben, eine pareto-optimale Lösung zu erwarten. Wenn die konkurrierenden Politiker gezwungen sind, ihre Budgetvorschläge gleichzeitig anzukiundigen,wird jede Partei ein Budget anbieten, das niemanden besserstellen kann, ohne einen anderen schlechterzustellen. Die verschiedenen Parteiprogramme unterscheiden sich allein durch ihren verteilungspolitischen Inhalt. 3 )

Dieses Ergebnis beruht auf folgendem Mechanismus: Jeder Politiker, der ein Budget anbietet, das paretosuboptimal ist, wird gegenüber einem anderen Politiker verlieren, der bei gleichem verteilungspolitischem Inhalt ein Programm anbietet, das eine optimale Lösung verspricht. Ein obergang von einer suboptimalen zu einer optimalen Lösung führt zu einem Oberschuß, auch wenn die Verlierer dieses Obergangs voll kompensiert werden. Mithin steht dieser entstandene Oberschuß dann zur Verteilung frei - und damit werden die Bürger, die diesen Oberschuß erhalten, für die diese optimale Lösung anbietenden Politiker stimmen, während die übrigen indifferent sind.

1) Dadurch soli das Paradoxon vermieden werden, das oft in der Literatur der ökonomischen Theorie der Politik zu finden ist: Nämlich daß bestimmte politische Entscheidungen ( $z$. B. über öffentliche Güter) bereits unter Annahme einer bestimmten Struktur anderer Größen ( $z$. B. Steuersätze) abgeleitet werden, obwohl diese Struktur selbst Ergebnis eines politischen Entscheidungsprozesses ist bzw. sein müBte.

2) DOWNS (1968) S. 178

3) vgl. zum folgenden SHUBIK (1968) 
Um die Wahrscheinlichkeit zu minimieren, von einer anderen Partei geschlagen zu werden, muß jede Partei ein paretooptimales Programm anbieten. In diesem Fall kann sie nämlich nur durch Programme mit einem anderen verteilungspolitischen Inhalt geschlagen werden, nicht jedoch auf Grund von im eigenen Programm enthaltenen Ineffizienzen. Damit minimiert sie die Anzahl der ihrem Programm überlegenen Alternativen, die andere Parteien zur Auswahl stellen können. Es spielt dabei keine Rolle, ob diese Alternativen tatsächlich zur Auswahl gestellt werden. Allein der Umstand, daB im Falle des Angebots eines suboptimalen Programms andere Parteien in die Lage versetzt werden, ein besseres und damit erfolgreiches Programm zur Wahl zu stellen, sorgt dafü, daß jede Partei nur optimale Lösungen anbietet. Ein Staatsversagen auf der Nachfrageseite kann unter diesen Voraussetzungen nicht zustande kommen.

Zu ähnlichen Ergebnissen kommt in einem etwas anderen Kontext KRAMER (1977). In seinem Modell wird nachgewiesen, daB in einem Zwei-Parteien-System eine Folge von Mehrheitsentscheidungen zu einer situation füht, in der nur noch allokationseffiziente Programme gegeneinander abgestimmt werden. Angenommen wird dabei, daß die jeweils siegreiche Partei bei dem Programm bleibt, mit dem sie in der letzten Wahl eine Mehrheit errungen hat, und die andere Partei ein neues Programm anbietet. Dabei soll letztere ihren Stimmenanteil maximieren, d.h. versuchen, möglichst viele Stimmen (und nicht nur die Minimalmehrheit, also $50 \%+1$ ) auf sich zu vereinigen.1) Sind die

1) Diese Annahme ist entscheidend. Wenn die Partei, die als letzte ihr Programm formuliert, nur eine (minimale) Mehrheit anstrebt, muB eine Folge von Wahlen nicht zu einem Paretooptimum konvergieren, es können sogar nicht-pareto-effiziente Programme gegeniiber paretoeffizienten eine Mehrheit erhalten. Vgl. dazu DOWNS (1968) S. $179 \mathrm{ff}$. und besonders MCKELVEY (1976). 
Präferenzen der Bürger bekannt, ist die jeweilige Oppositionspartei immer in der Lage, ein Programm zu formulieren, das eine Mehrheit findet. Insgesamt wird es jedoch unabhängig vom Ausgangspunkt nach einer Reihe von Wahlen dazu kommen, daB nur noch pareto-optimale Programme zur Auswahl stehen. In diesem $F$ all ist Staatsversagen nur eine vorübergehende Erscheinung.

Vergleicht man das Ergebnis mit den in Abschnitt 3.1 abgeleiteten Voraussetzungen des Staatsversagens, erkennt man folgendes: Zwar ist das Zielsystem der Politiker von dem der Bürger verschieden- ihr Ziel ist es ja, die Wahlen zu gewinnen, nicht eine optimale Versorgung der Bürger mit öffentlichen Leistungen zu gewährleisten. Durch die Abstimmung selbst haben die Burger aber ein geeignetes Instrument in der Hand, um $z$ wischen verschiedenen Budgetvorschlägen zu diskriminieren und so eine Ausrichtung des Budgets auf ihre Präferenzen zu erzwingen. Eine Partei, deren Programm nicht den Bürgerpräferenzen entspricht, kann auf die Dauer niemals die Wahl gewinnen. Ein Staatsversagen kann in diesem Modell vollkommener politischer Konkurrenz, außer für die Dauer eines Anpassungsprozesses, nicht auftreten.1)

Allerdings ist unter diesen Umständen nicht immer ein konsistentes Abstimmungsergebnis zu erwarten. Mehrheitsentscheidungen durften in aller Regel instabil sein, d.h. es gibt keine Alternative, die allen anderen bei einer Wahl vorgezogen wird. Das Problem der Nichtexistenz eines Gleichgewichts in einem durch Mehrheitswahl charakterisierten EntscheidungsprozeB wurde bereits von ARROW (1962) beschrieben und führt zu Phänomenen wie zyklischen Mehrheiten bzw. einer Instabilität des Ergebnisses politischer Prozesse. Im Extremfall kann es bei mehr als

i) HERDER-DORNEICH's "List der Demokratie"; vgl. HERDER-DORNEICH (1959) S. $58 \mathrm{f}$. 
zwei gleichzeitig zur Abstimmung stehenden Programmen zu der Situation kommen, daB keine der Alternativen eine Mehrheit erhält; - bei parweiser Abstimmung kann jede Alternative (je nach Reihenfolge der Abstimmung) gewinnen.

Die Existenz eines Gleichgewichts kann nur unter stark einschränkenden Voraussetzungen über die Form der individuellen Präferenzen bzw. der Verteilung der Individuen mit verschiedenen Präferenzen nachgewiesen werden, Einschränkungen, deren Erfüllung in der Realität recht unwahrscheinlich ist. 1)

Die Nichtexistenz eines Gleichgewichts im politischen AbstimmungsprozeB ist dann immer augenfällig, wenn Verteilungsfragen angesprochen werden.2) Unter diesen Umständen kann jede pareto-optimale Verteilung eine Mehrheit finden, und das Ergebnis des politischen Prozesses bleibt unbestimmt.

1) Zum Beispiel Eingipfligkeit der Präferenzen, vgl. BLACK (1958). KRAMER (1973) gibt eine Obersicht über die notwendigen Annahmen für die Existenz eines Gleichgewichts und kommt zu dem Ergebnis, daB "all exclusion conditions for equilibrium under majority rule are extremely restrictive in the sense that they are incompatible with even a very modest degree of heterogenity of tastes" ( $S .269$ ). Tatsächlich unterliegen die meisten Ansätze, die Bedingungen für eine Stabilität von Mehrheitsentscheidungen aufzeigen, Annahmen, die darauf hinauslaufen, daß die Präferenzen der Individuen nicht allzu stark voneinander divergieren dirfen, oder bestimmten Annahmen über die Symmetrie der Präferenzverteilung iuber die Individuen, wie z.B. bei PLOTT (1967). Insgesamt durften die getroffenen Annahmen allgemein zu unrealistisch sein, um der Hoffnung auf stabile Mehrheitsentscheidungen eine Grundlage $z u$ geben. Siehe dazu auch die Obersicht bei MUELLER (1979) und die dort zitierte Literatur.

2) vgl. dazu KNAPPE (1981) 


\subsubsection{Die Bedeutung der Information}

Alle bisherigen Ergebnisse hingen von der Voraussetzung $a b$, daB Politiker und Wähler über vollkommene Informationen verfügen bzw. die Informationsbeschaffung selbst ein kosten- und zeitloser ProzeB ist. Diese Annahme kann aber nicht aufrechterhalten werden.

Für die beschriebenen Zusammenhänge sind Informationen auf folgenden Ebenen wichtig: 1 )

1. Die Wähler müssen die verschiedenen Parteiprogramme genau kennen. Erst dann ergibt sich ein $Z$ wang für die Politiker, sich mit ihren Budgetvorschlägen den Wählerpräferenzen anzupassen, nur unter diesen Umständen ist eine pareto-optimale Versorgung zu erwarten. Die Aufgabe der Annahme eines vollkommen informierten Wählers gibt den jeweils um die Regierung streitenden Politikern einen gewissen Spielraum, von den Wählerpräferenzen abzuweichen, ohne Risiko zu laufen, dadurch Einbußen an Wählerstimmen zu erleiden.

2. Die Politiker müssen über die Präferenzen der Wähler informiert sein. Nur in diesem $F$ all besteht eigentlich die Möglichkeit, daB die Parteien ihre Angebote auf die Präferenzen der Wahlbürger abstellen. Bei einem limitierten Angebot an verschiedenen politischen Programmen, deren Zustandekommen nur auf unvollkommene Information zurückzuführen ist, kann der rationale Wähler sich zwar für das am wenigsten schlechte Programm entscheiden, eine vollkommene Obereinstimmung $z$ wischen Angebot an staatlicher Leistung und Nachfrage der Wähler ist jedoch Zufall. Es ist allerdings zu prüfen, ob der WahlprozeB selbst nicht die notwendigen Informationen vermitteln kann.

i) vgl. BERNHOLZ (1969) 
3. Eine wesentliche Problematik liegt allerdings auch in der Interpretation des Zusammenhangs von öffentlicher Leistung und dem Ergebnis staatlichen Handelns. Eine rationale Entscheidung über jede alternative Staatstätigkeit setzt eine richtige Vorstellung über die Ergebnisse dieser Maßnahmen voraus. Bisher wurde unterstellt, daB die Wähler iber die verschiedenen erreichbaren Zustände der Gesellschaft entscheiden. Zur Entscheidung angeboten werden aber nur verschiedene Mittel (Steuersätze, Staatsausgaben usw.), die diesen Zustand herstellen sollen. Anders ausgedrijckt: Die jeweiligen Politiker haben keine direkte Kontrolle über den Output der Statstätigkeit, sondern nur über die Inputs, die diese Zustände herstellen sollen. Dies ist solange unproblematisch, wie der Zusammenhang Input und output eindeutig und bekannt ist. Tatsächlich ist dies aber nicht so, wie die umfangreiche Literatur zu den Problemen wirtschaftspolitischer Beratungen zeigt. Damit wird das Wahlproblem aber heikel. Selbst wenn die Wähler die Ergebnisse des politischen Handelns kennen, der Zusammenhang zwischen diesen Ergebnissen und der Ursache aber nicht bekannt ist, ist eine rationale Diskriminierung $z$ wischen verschiedenen Alternativen $n i c h t$ möglich, und nur auf Grund von unvollständigen und falschen Einschätzungen machbar. Das gilt auch in umgekehrter Richtung, wenn die Politiker die Ergebnisse ihrer Handlungen nicht kennen.

Auf die einzelnen Punkte soll im folgenden näher eingegangen werden.

Der geringe Informationsstand der Wähler wurde bereits von SCHUMPETER beschrieben und beklagt. DOWNS hingegen versuchte den geringen Informationsstand der Wähler als Ergebnis eines durchaus rationalen Handelns zu begreifen. 
Die Grundlagen sind folgende: 1 ) Ausgangspunkt ist eine Situation, in der der einzelne Wahlbuirger keine Informationen uber die zur Wahl stehenden Programme hat. Um an diese Informationen zu gelangen, muB er in einen mit Kosten verbundenen SuchprozeB eintreten. Diese Kosten können aus monetären Ausgaben bestehen, aber auch als nichtmonetäre Aufwendungen (Zeit etc.) auftreten. Dabei müBten die Wähler nicht nur Informationen über den Inhalt der ihnen zur Auswahl stehenden Programme sammeln, sie müBten auch die Auswirkungen dieser Programme auf ihre eigene Situation zu bestimmen versuchen.

Diesen Aufwendungen gegenüber stehen die (erwarteten) Kosten, die der Wähler auf sich nehmen muß, wenn er nicht oder nur unvollständig informiert ist und auf Grund dieser mangelnden Informationen eine falsche Wahl trifft.2) Dabei diurten diese erwarteten kosten von einer Reihe von Faktoren abhängen. Genannt wird in der Literatur z.B. die Höhe des Parteiendifferentials. 3 ) Je größer der erwartete Unterschied $z$ wischen den verschiedenen Parteiprogrammen ist, desto höher sind die Verluste, die man dadurch in Kauf nimmt, daß die "falsche" Partei gewinnt, und desto wichtiger wird die eigene Entscheidung.

Von wesentlicher Bedeutung ist auch die Anzahl der Mitwähler,4) da mit steigender Anzahl derer das Gewicht der eigenen Stimme abnimmt und somit die erwarteten Kosten einer falschen Entscheidung relativ gering sind.

\footnotetext{
i) vgi. BARTLETT (1973) S. $27 \mathrm{ff}$.

2) Diese "Kosten" können gegebenenfalls auch entgangene Erträge sein.

3) DOWNS (1968) S. $234 \mathrm{ff}$.

4) TULLOCK (1967), Kapitel 7
} 
Ebenfalls dïrfte die Vermutung über die Differenz der Stimmenzahl zwischen den zur Wahl stehenden Programmen relevant sein, da bei einem Kopf-an-Kopf-Rennen ebenfalls die Bedeutung der eigenen Stimme und damit einer falschen Entscheidung zunimmt. 1 )

Ein "Informationsgleichgewicht" fur den Wähler ergibt sich dann da, wo die Grenzkosten des Sammelns und Verarbeitens weiterer Informationen gleich sind dem (erwarteten) Grenzertrag besserer Informiertheit. 2)

Folgende Graphik soll dies verdeutlichen: 3 )

1) Diese oberlegung liegt auch den zahlreichen Ansätzen zugrunde, die sich mit den Bedingungen, die zu einer Wahlbeteiligung überhaupt führen, befassen. Auch hier ist der EinfluB der eigenen Stimmabgabe auf das Ergebnis ein wichtiger Faktor, der bei der Entscheidung Wählen oder Nicht-Wählen herangezogen wird. Vgl. DOWNS (1969), Kap. 18, RIKER/ORDESHOOK (1968). Den Zusammenhang $z$ wischen Informationsstand und Wahlbeteiligung beleuchten Z.B. TOLLISON/WILLETT (1973), TOLLI SON/CRAIN/PAUTLER (1975)

2) Nicht ibbersehen werden soll dabei das Problem, daB Informationen über die politischen Programme erst in einem kostspieligen Prozeß beschafft werden müssen, die Grenzerträge der zu erwerbenden Informationen aber bekannt bzw. als Erwartungsgrößen gegeben sind. Diese Grenzerträge sind ja ohne Kenntnis der Auswirkungen alternativer Programme, also der unbekannten Größen, gar nicht zu bestimmen. Dieses Problem sei im folgenden jedoch vernachlässigt, ebenso wie alle Probleme, die sich aus einer möglichen "offentlichen-Guts"-Eigenschaft der Information ergeben.

3) siehe BARTLETT (1973) S. 30 

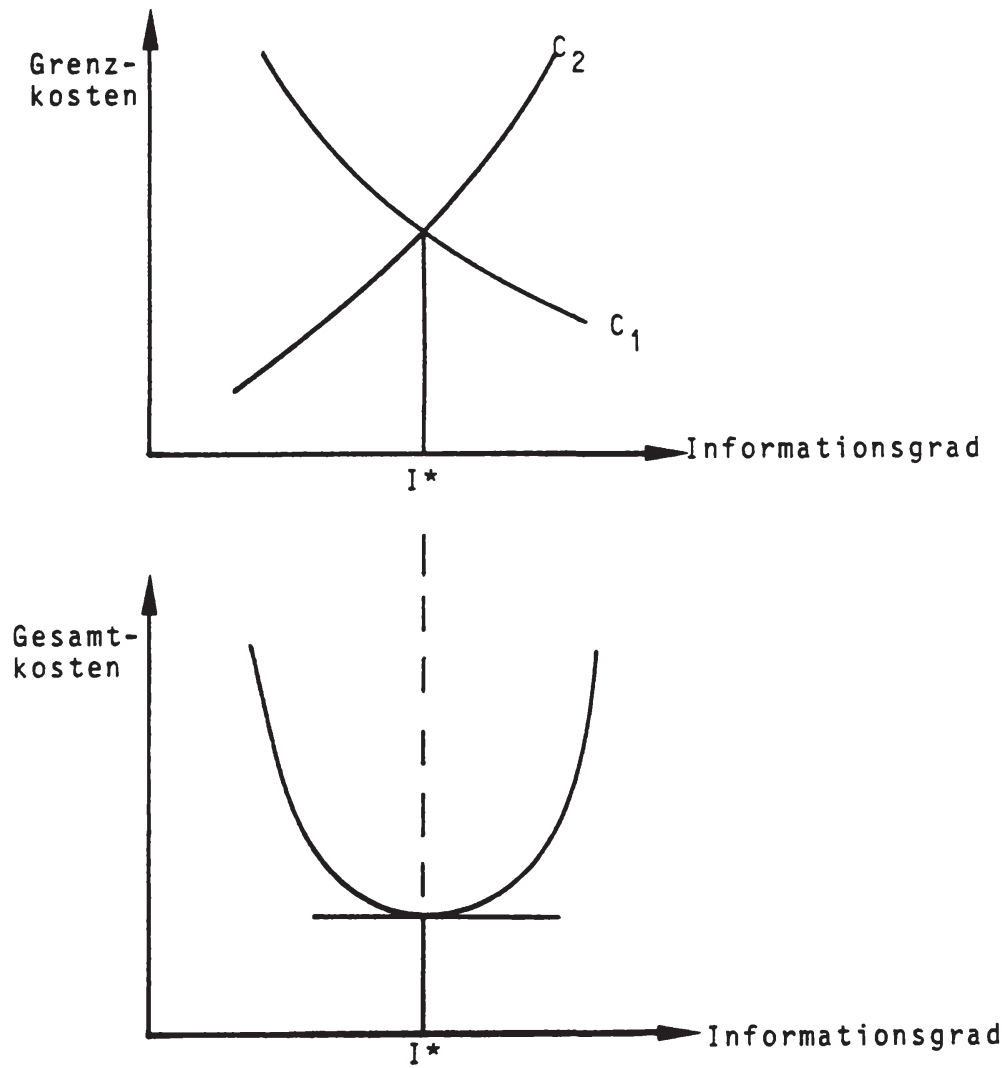

Die Kurve $C_{2}$ symbolisiert den Verlauf der Grenzkosten der Informationsgewinnung ("Costs of becoming informed"), $C_{1}$ die marginalen Kosten, die durch fehlende Informationen und dadurch bedingte falsche Entscheidungen entstehen ("Costs of being uninformed"). Ein optimaler Informationsstand ergibt sich dann bei I*, bei dem zugleich (vom 
individuellen Standpunkt gesehen) die Gesamtkosten der Informiertheit minimal sind. Damit ist die Höhe des Grades der "rationalen Informiertheit" (oder "rationalen Uninformiertheit") des Wählers bestimmt.

Dabei dürte die Höhe des optimalen Informationsstandes nicht als allzu groB veranschlagt werden. Gerade der geringe EinfluB der eigenen Wahlentscheidung läBt die Erträge der Information relativ gering erscheinen (wenigstens soweit es nur um die Wahlentscheidung geht), die Höhe der Informationskosten hingegen diurfte bei relativ komplizierter Materie nahezu prohibitiv wirken.1)

Anzumerken ist, daB der optimale Informationsstand sehr verschieden sein kann, sowohl was die Höhe der Informiertheit bestimmter Wählergruppen betrifft als auch was den Grad der Information über spezielle Teilbereiche des politischen Angebots angeht. Der optimale Grad der Informiertheit ist weder bei allen wählern noch bei allen zur Wahl gestellten Detailproblemen der gleiche. Die Bedeutung einer ungleichen Verteilung von Information ist noch herauszuarbeiten.

Selbst wenn die Wähler über die Inhalte der verschiedenen Programme und deren Auswirkungen informiert sind und sich rational entscheiden, ist immer noch offen, inwiefern das Angebot der Leistungen ihren Wünschen entspricht. Im Fall der vollkommenen politischen Konkurrenz wurde gesagt, daB eine optimale Anpassung des politischen Programms an die Wählerpräferenzen deren Kenntnis voraussetzt. Besteht diese nicht, dann ist das Angebot an Programmen nicht auf die Wähler abgestimmt.

i) vgl. zum Informationsstand von Wählern BERNHOLZ (1975) S. $81 \mathrm{ff}$. 
Für die Politiker stellt sich somit tendenziell dieselbe Problematik wie für die Wähler. Sie haben zu entscheiden, inwieweit sie sich auf eine kostenträchtige Suche nach Informationen über die Präferenzen der Wähler machen, wobei sie die Wahrscheinlichkeit, eine Wahl dadurch zu verlieren, $d a B$ sie (im Vergleich zu den politischen Konkurrenten) ein den Wählerwijnschen nicht angepaßtes Programm anbieten, bewerten und gegen die Kosten der Informationsgewinnung abwägen müssen. Auch hier gibt es einen optimalen Informationsstand, der nicht vollkommene Informationen umfaßt und daher nicht alle Wählerpräferenzen perfekt berücksichtigt.

Allerdings wïrde eine Unsicherheit der Politiker über die Wählerpräferenzen zu keinen Problemen führen, wenn der WahlprozeB selbst ein effizienter Mechanismus zur Diskriminierung $z$ wischen verschiedenen Alternativen darstellen und damit zur Aufdeckung der Präferenzen der Bürger führen wïrde. Selbst wenn die Politiker keine Informationen über die wähler haben, dann werden die Wähler doch immer für das Programm stimmen, das ihren Präferenzen am nächsten kommt. Der Politiker gewinnt die Wahl, der "zufällig" das richtige Programm anbietet -alle anderen fallen durch. Vernachläsigt man die Kosten der Formulierung des politischen Angebots, dann ist eine Kenntnis der Wählerpräferenzen nicht unbedingt notwendig, um zu einem optimalen Ergebnis zu kommen. Der Abstimmungsmechanismus selbst ist ein effizientes system zur Diskriminierung und ruft damit Lernprozesse hervor, die die Politiker über die Präferenzen der Bürger per Wahlentscheidung informieren. In einem dynamischen ProzeB ist dann damit $z$ u rechnen, daß langfristig jedes neu gewählte Programm eine Verbesserung darstellt, bis eine optimale Anpassung an die Wählerwünsche erreicht ist.1)

i) vgl. BERNHOLZ (1975) S. $36 \mathrm{ff}$. 
Eine solche Oberlegung abstrahiert aber von allen Eigentiumlichkeiten des Wahlprozesses in einer Demokratie. Folgende Eigenschaften sind dabei zu bedenken:

Die angesprochene Lösung funktioniert nur dann, wenn die optimale Alternative auch irgendwann in den zur wahl gesteliten Angeboten enthalten ist. Dies setzt aber letztendlich eine unendliche Anzahl von Angeboten voraus, die in der Realität nicht gegeben ist und auf Grund der dann entstehenden hohen Entscheidungskosten auch nicht gegeben sein kann. Die Wahl besteht immer zwischen einer begrenzten Anzahl von Alternativen, von denen die vielleicht am wenigsten schlechte gewählt wird. Alternativ könnte angenommen werden, daB die Wahlen unendlich oft wiederholt werden. Auch dies dürte in der Realität kaum vorzufinden sein und auf Grund der mit Wahlen verbundenen Kosten auch nicht wünschenswert sein.

Aber selbst wenn die Menge der zur Wahl stehenden Alternativen unbegrenzt ist, kommt man damit zu keiner eindeutigen Entscheidung. Bei mehreren Alternativen ist in der Regel nicht zu erwarten, $d a B$ eine davon eine Mehrheit erhält - somit ist das Ergebnis des Wahlprozesses zwischen einer unendlichen Anzahl von Alternativen unbestimmt. Im Prinzip kann (zumindest auf Verteilungsebene) jedem Wähler eine Alternative zugeordnet werden, die er für die beste hält. Eine Entscheidung ist aber nur möglich, wenn (im Falle der Mehrheitsregel) ein Programm die absolute Mehrheit erhält. Die Tendenz zur Begrenzung der Anzahl der angebotenen Alternativen wird dadurch gesteigert, ja notwendig, da eine höhere Anzahl von Angeboten eine Mehrheitsfindung erschwert. Eine Beschränkung der Anzahl der zur Auswahl stehenden Alternativen kann daher als Mittel interpretiert werden, um $z u$ einer eindeutigen Entscheidung zu gelangen - mit den angefiuhten negativen Effekten auf den Informationsgehalt von Wahlergebnissen. 
Zudem kann in einer Mehrheitswahlentscheidung auch ein suboptimales Programm aufgrund seines verteilungspolitischen Inhalts gegenüber einem optimalen gewinnen. Voraussetzung dafur ist, $d a B$ das suboptimale Programm eine Mehrheit der Wähler auf Kosten der Minderheit bevorteilt. $D a B$ es dann möglicherweise noch ein drittes Programm gibt, das wiederum alle besser stellen könnte, läßt sich aus der Wahlentscheidung nur dann ableiten, wenn bekannt ist, auf welche spezifischen Unterschiede der Wahlsieg zurückzuführen ist. In einer repräsentativen Demokratie ist es jedoch nicht üblich, daß alle Einzelentscheidungen getrennt zur Wahl gestellt werden. Letztendich stehen dem Wähler nur verschiedene "Pakete" zur Auswahl, in denen eine Vielzahl von Punkten enthalten ist. Weichen diese Pakete in mehreren Punkten voneinander ab, ist nicht mehr eindeutig identifizierbar, auf welche speziellen Unterpunkte ein Wahlsieg oder eine Wahlniederlage zurückzufuhren ist. Der Informationsgehalt der reinen Wahlentscheidung ist entsprechend gering, die Einstellung der Wahlbürger gegenüber den einzelnen Programmpunkten ist aus der Wahlentscheidung nicht abzuleiten.1)

Der bereits erwähnte Aspekt, daß die Wahlzeitpunkte weit auseinander liegen, d.h. daB nicht unendlich oft gewählt wird, bringt ein weiteres Problem mit sich. Für alle Probleme und Maßnahmen, die zwischen zwei Wahlzeitpunkten stattfinden, läBt sich keine Wahlentscheidung durchführen. In diesem Fall ist der Politiker darauf angewiesen, zu raten, welche Entscheidung der Wähler wohl getroffen hätte, wenn inm diese Alternative zur Wahl gestellt worden wäre. Eine Diskriminierung durch einen tatsächlich stattfindenden Wahlvorgang ist dann aber nicht gegeben.

i) $\mathrm{Vgl.BRETON}(1974)$ S. $50 \mathrm{f}$. 
Aus dem Gesagten folgt, daB der politische ProzeB durch Unvollkommenheiten geprägt ist, die eine Abweichung von den unter dem Regime der vollkommenen politischen Konkurrenz abgeleiteten Ergebnissen implizieren. Die Annahme vollständiger Information als Voraussetzung für eine optimale Nachfrageentscheidung ist nicht realistisch, und der AbstimmungsprozeB selbst ist nur begrenzt geeignet, die notwendigen Informationen zu vermitteln.

3.3.3 InteressengruppeneinfluB und unvollständige Information

Durch die fehlenden Informationen ergibt sich somit ein gewisser Handlungsspielraum auBerhalb des kompetitiven Wahlprozesses, dessen Auswirkungen untersucht werden miussen. Einerseits besteht ein gewisses Informationsdefizit der Wähler über Inhalt und Auswirkungen ihrer eigenen politischen Entscheidung, so daß ein von den Wählerpräferenzen abweichendes Parteiprogramm nicht unbedingt zum Verlust der Wahlen führen muß. Andererseits besteht auf Seiten der Politiker ein Defizit an Kenntnissen über die Wählerpräferenzen und die Auswirkungen ihrer Maßnahmen auf das Wahlergebnis.

Gegenüber der Lage vollkommener Information ergibt sich daraus als wesentliche Folgerung, daß die Wahlentscheidung nicht die einzige (und vermutlich nicht einmal die bedeutendste) Methode der EinfluBnahme von Privaten auf die staatlichen Entscheidungen ist. Die Politiker verfügen über einen bestimmten Handlungsspielraum und können auch Maßnahmen durchsetzen, die in einer Wahlentscheidung unter vollkommener Information nicht möglich gewesen wären. Daher besteht für die Mitglieder der Gesellschaft ein 
Anreiz, politische Entscheidungen durch andere Mittel als über Wahlen zu beeinflußen. Oberlegungen dieser Art sind die Grundlage für die "Interessengruppen-Theorie des Staates"1) bzw. fuir Ansätze, die sich mit dem EinfluB organisierter Gruppen auf die politische Entscheidung befassen.2)

Ausgangspunkt sind dabei die Oberlegungen OLSONS (1965) zur Gründung von Organisationen: Diese werden dann gebildet, wenn es gemeinsame Interessen gibt, die die Individuen einzeln weniger gut durchsetzen können als in einem kollektiven Verband. Dabei ist die Organisationsfähigkeit von Gruppeninteressen von verschiedenen Faktoren abhängig. Wichtig sind dabei insbesondere die Möglichkeiten, die die einzelne Organisation hat, um Nichtmitglieder von den Vorteilen, die sie erlangt, auszuschließen. Wenn es möglich ist, die Vorteile einer organisation in Anspruch zu nehmen, ohne ihr anzugehören und Beiträge zu leisten, wird es zum Phänomen des "Free-Rider"-Verhaltens kommen: Statt der Organisation beizutreten, werden die Individuen versuchen, ohne Eigenbeteiligung in den Genuß der von der jeweiligen Organisation bereitgestellten Leistungen zu kommen. Ein solches Verhalten kann die Entstehung einer Organisation gegebenenfalls sogar verhindern, wenn $z u$ viele potentielle Mitglieder die Free-Rider-Position einnehmen. Dabei dürten die Ausschlußmöglichkeiten durchaus ungleich sein, nicht alle Interessen durften in gleichem Maße organisatorisch vertretbar sein.

1) vgl. MCCORMICK/TOLLISON (1981); aus der umfangreichen Literatur zum E influB von Verbänden s. z.B.: LIEFMANN-KEIL (1969), ROSE-ACKERMANN (1980), BERNHOLZ (1969) (1975) und $v$. BEYME (1980)

2) Wobei die "Gruppe" auch aus nur einem einzigen Individuum bestehen kann, das EinfluB auf den politischen Prozeß nimmt. 
Die Einflußnahme durch kollektive Organisationen ist dabei nicht kostenlos. Damit die Gruppen Einfluß nehmen können, müssen sie sich organisieren, d.h. als Gruppe konstituieren. Dadurch entstehen bereits Kosten, da die Aufstellung und Erhaltung einer Organisation bereits Ressourcen verschlingt. Weiterhin muissen zur EinfluBnahme selbst Ressourcen eingesetzt werden.

Im wesentichen beruht dabei die Macht der Verbände oder spezieller Interessengruppen (Lobbies) auf einem unterschiedlichen Informationsniveau über die Interessen von organisierten und nichtorganisierten Individuen. Zu unterscheiden ist dabei wieder $z$ wischen den beiden Ebenen der Informiertheit:

a) Verbände sind Organisationen, die sich aus Mitgliedern mit einer gemeinsamen zielrichtung bzw. einem gemeinsamen, partikulären Interessengebiet zusammensetzen. Sie beschaffen Informationen über die staatlichen Maßnahmen, soweit sie die Interessen dieser spezifischen Gruppe tangieren bzw. Auswirkungen auf die Angehörigen dieser Gruppe haben könnten, und geben diese Informationen an die Mitglieder weiter. Daraus folgt, daß Interessengruppen, die über eine Organisation verfügen, im allgemeinen über die sie betreffenden speziellen Regelungen und Auswirkungen statlicher Maßnahmen besser informiert sind als die übrigen Mitglieder der Gesellschaft, aber nicht besser über die sie nicht direkt betreffende Maßnahmen des Staates. Das Ergebnis ist, daß statiche Entscheidungen, die spezifische, durch organisierte Interessengruppen verbundene Individuen betreffen, zu größeren Wählerverlusten oder -gewinnen führen als Maßnahmen, die die Individuen auf Gebieten treffen, in denen Interessen 
nicht organisiert sind. Daraus ergibt sich die altbekannte These von der Bevorzugung spezifischer Interessen auf Kosten des Allgemeininteresses.1)

b) Interessengruppen vermitteln Informationen an die Wähler. Dabei werden sie ein selektives Angebot an Informationen an die Stimmbürger weitergeben, d.h. die Informationen, die den Wähler davon überzeugen, daB die für die Interessengruppe vorteilhaften Maßnahmen auch für sie von Nutzen sind, werden verbreitet, gegenteilige Informationen zurückgehalten. Verbände betreiben somit "Reklame" für ihre Ziele. Neben der "Subventionierung" spezifischer Informationen2) haben Interessengruppen natülich auch die Möglichkeit, systematisch falsche Informationen unter die Wähler zu streuen und damit EinfluB auf den politischen EntscheidungsprozeB $z u$ nehmen. Insgesamt führt die Aktivität von Interessengruppen zu einem veränderten Wahlverhalten, das den stimmenmaximierenden Politiker zu bestimmten, die Interessengruppen begünstigenden Maßnahmen veranlaßt.

c) Weiterhin vermitteln Interessengruppen den Politikern die Informationen über die Präferenzen ihrer Mitglieder und die Auswirkungen der MaBnahmen auf das durch sie vertretene Wählerpotential, die sonst nicht verfügbar wären. Dabei spielt keine Rolle, ob Interessengruppen die wahren Präferenzen ihrer Mitglieder aufdecken oder diese falsch darstellen.

1) Das heiBt nicht unbedingt, $d a B$ damit eine ineffiziente Situation per se gegeben ist. Wenn die Kosten, herauszufinden was das Allgemeininteresse ist, wirklich extrem hoch sind, dann kann es durchaus rational sein, darauf keine Rücksicht zu nehmen.

2) siehe BARTLETT (1973) S. 71 
Es ergibt sich aus diesem Grunde, daß die Politiker eher den Wuinschen der durch organisierte Interessengruppen vertretenen Bürgern entsprechen werden als den Wünschen der nichtorganisierten. Dem Politiker wird klargemacht, welche Wählerstimmen er auf jeden Fall nicht erhält, wenn er irgendwelche, spezifische Gruppen begünstigende oder benachteiligende MaBnahmen trifft oder nicht. Bei Entscheidungen, die nur nichtorganisierte Bürger betreffen, ist das Ergebnis hingegen ambivalent und nicht eindeutig.

Die Tätigkeit von Interessengruppen muB solange zu keiner systematischen Verzerrung von Entscheidungen führen, wie die Bürger sich gleichmäBig in Interessengruppen organisieren und ihre Präferenzen mit gleicher Intensität an die Politiker weitergeben können. Interessengruppen sind dann möglicherweise nur rationale Institutionen, um das angesprochene Informationsdefizit zu verkleinern. Erst eine ungleichmäßige Repräsentation der Bürger durch verschiedene Interessengruppen, die zum Beispiel auf unterschiedliche kosten der Organisation zurückzuführen ist, führt zu einer Abweichung des letztendlich getrof fenen Entscheidungspakets von dem, das bei einer reinen Wahlentscheidung getroffen worden wäre. In diesem Sinne erhalten die Bürger, deren Interessen durch Organisationen vertreten werden, quasi ein stärkeres Gewicht als die Bürger, bei denen das nicht zutrifft.

Allerdings ist der unterschiedliche Informationsgrad von organisierten und nichtorganisierten Bürgern nicht der einzige Grund, aus dem ein höherer $E$ influB von Verbänden abgeleitet werden $k a n n$ und wird. Interessengruppen können auch dadurch EinfluB nehmen, indem sie für ein gleichgerichtetes Handeln ihrer Mitglieder sorgen und dies als Druckmittel in den politischen ProzeB einsetzen.1) obt

i) vgl. BERNHOLZ (1975), V. BEYME (1980) 
eine Interessengruppe eine gewisse Macht aus und kann durch eigene Aktionen andere erheblicher schlechter stellen, ist sie in der Lage, ihr genehme politische Entscheidungen $z$ u erpressen (z.B. Gewerkschaften durch Drohung eines "politischen" Streiks, das Offizierskorps durch Putschandrohung, die Mafia durch Mordankündigungen). Man kann eine solche Situation als Vermachtung der politischen Märkte verstehen mit dem Ergebnis, daß die Konkurrenz um Wählerstimmen von untergeordneter Bedeutung ist.

Daneben gibt es aber auch andere Mechanismen, die zu einer Dominanz der politischen Entscheidungen durch Interessengruppen führen können und die auf der organisiertheit dieser Gruppen beruhen. TULLOCK ${ }^{1}$ ) hat in mehreren Beitrögen auf die Möglichkeit hingewiesen, daß der Mechanismus des Stimmentausches, des "log-rolling", zu Ergebnissen des politischen Prozesses führen kann, bei denen Minderheitsinteressen dominieren. Unter Stimmentausch versteht man, $d a B$ eine Wählergruppe bei bestimmten Entscheidungen, die für sie relativ unwichtig sind, zugunsten einer anderen Gruppe für eine Maßnahme stimmt, die ihrem eigenen Interesse zuwider läuft. Dafür stimmt dann die andere Gruppe im Interesse der ersten, wenn es um Entscheidungen geht, die für die erste Gruppe relativ wichtig sind. Die Wähler der einen Gruppe tauschen sozusagen ihre Stimmen bei der einen Entscheidung gegen die Stimmen der anderen bei einer anderen Entscheidung.

Eine gewisse Problematik im Stimmentausch-Konzept ergibt sich aus dem Umstand, daß in der Regel Situationen, in denen es zu einem Stimmentausch kommen kann, durch die

1) TULLOCK (1959) (1970), BUCHANAN/TULLOCK (1962), Kap.
IOff. 
Existenz zyklischer Präferenzen1) gekennzeichnet sind. Die jeweils existierende Minderheitskoalition ist nicht die einzig mögliche, es können sich auch andere Gruppen zu einem Stimmentausch zusammenfinden - unter Umständen unter EinschluB einer der an der bestehenden Koalition beteiligten Gruppen. Ergebnis ist ein instabiler politischer ProzeB. 2)

Diese Situation kann dadurch geändert werden, $d a B$ organisierte Interessengruppen auftreten. Dadurch, daB sie organisiert sind, können sie bindende Vereinbarungen mit anderen Interessengruppen eingehen und damit durch einen expliziten Stimmentausch zu einer Stabilisierung des politischen Prozesses beitragen. ${ }^{3}$ )

Die stabilisierende Funktion ergibt sich vor allem deshalb, da die am Log-rolling-Prozeß beteiligen Gruppen uber mehrere Perioden bestehen bleiben, d.h. selbst stabile Partner eines Stimmentauschprozesses sind, und damit ein konstantes Verhalten von diesen erwartet werden kann, während die nichtorganisierten wähler kaum als Partner einer über mehrere Perioden bindenden Vereinbarung in Frage kämen. Ein Interesse an der Aufrechterhaltung einer bestimmten Situation, auch wenn kurzfristig ein besseres Ergebnis erzielt werden könnte, ergibt sich ja nur, wenn die langfristigen Erträge höher sind als die kurzfristigen. Das setzt voraus, daB die Partner des Stimmentauschprozesses uber mehrere perioden hinaus Bestand haben.

\footnotetext{
1) BERNHOLZ (1974) (1976)

2) vgl. dazu Abschnitt 3.3.1.

3) BERNHOLZ (1977)
} 
Im folgenden soll dieser Aspekt aber keine weitere Berücksichtigung finden. Hier interessieren in erster Linie die Auswirkungen des unterschiedlichen Informationsstandes.

\subsubsection{Politischer EinfluB und Staatsversagen}

Die mangelnde Information führt dazu, daß organisierte Interessengruppen auBerhalb von Wahlen Einfluß auf die Ergebnisse des politischen Prozesses nehmen können. Geht man dabei von eigennutzorientiertem Verhalten aus, so werden die Interessengruppen ihren $E$ influß im wesentlichen darauf richten, Einkommensvorteile für ihre Mitglieder zu erzielen. Diese Gewinne aus dem politischen Prozeß dürften regelmäßig zu Lasten anderer Gruppierungen gehen.1)

Diese Art von EinfluBnahme wird of bezeichnet als "rent-seeking" im Gegensatz zum "profit-seeking" durch produktive Tätigkeiten. 2) Als "Renten" sollen dabei jene Erträge bezeichnet werden, die die über den normalen opportunitätskosten eines Ressourceneinsatzes liegen.

"Profit-seeking" bezeichnet dabei den Einsatz von Ressourcen in einen produktiven Prozeß, dessen Ergebnis eine für die Gesellschaft nützliche Aktivität darstellt. Renten entstehen auch im normalen MarktprozeB, beispielsweise als obergangsphänomen, bevor ein Markt zum langfristigen

i) vgl. das Buch von MCCORMICK/TOLLISON (1981)

2) zur Theorie der rent-seeking society vgl. STIGLER (1971), POSNER (1971) (1974), PELTZMAN (1976), TOLLISON (1982), Beiträge in BUCHANAN/TULLOCK/TOLLISON (1980) 
Gleichgewicht gefunden hat. In diesem Fall erfiullen "Renten" eine positive ökonomische Funktion, indem sie Ressourcen in die relativ unterversorgten Sektoren locken.

"Rent-Seeking" bezeichnet einen etwas anders gelagerten Versuch, Gewinne zu machen. Hier interessiert vielmehr die Möglichkeit, künstlich eine Situation zu schaffen, in der die Erzielung einer Rente im oben definierten Sinn ermöglicht wird. "Rent-seeking" wird damit $z u$ einem Prozeß, der keine allokationsverbessernde Funktion hat und nur im guinstigsten Fall paretounschädlich ist. In der Regel durfte das Erzielen einer Rente mit einer ineffizienten Situation verbunden sein.

"Natürlicher" Adressat von Interessengruppen, die an der Erzielung einer Rente interessiert sind, ist der staat, der ja die Möglichkeit hat, bestimmte Zwänge auszuüben und somit Umverteilungsmaßnahmen zu organisieren, die nicht aus einem marktwirtschaftlichen WettbewerbsprozeB resultieren. So wurde die Theorie des "Rent-Seeking" auch im Zusammenhang mit der Analyse der Bestimmungsgrüde von bestimmten marktregulierenden Maßnahmen des Staates, wie Importkontrollen, Marktzutrittsbeschränkungen, Preisbestimmungen usw.1) Hier zeigte sich als erstes die Bedeutung der Einflußnahme von Interessengruppen, die uiber statiche Maßnahmen private Umverteilungsgewinne für ihr Klientel zu erzielen suchten. Die Ergebnisse können aber ohne weiteres auch auf andere statiche Maßnahmen ausgeweitet werden.2)

1) vgl. STIGLER (1971), POSNER (1974)

2) dazu PELTZMAN (1976), auf Steuerreformen bezogen FOLKERS (1983b) 
3.3.4.1 Politischer EinfluB und prozessuales Staatsversagen

Die Einflußnahme auf politische Entscheidungen ist kein kostenloser ProzeB. Die Höhe des politischen Einflusses kann dabei beschrieben werden durch eine Art Produktionsfunktion, deren Inputs die zur Einflußnahme aufgewandten realen Ressourcen darstelien.1)

Für die Interessengruppen unterliegt dabej die "Produktion" von politischem Einfluß ähnlichen Kalkülen wie eine normale Investitionsentscheidung. Sie haben abzuwägen, inwieweit weitere Ausgaben zur Erhöhung des eigenen Einflusses durch die pekuniären Erträge dieses Einflusses noch gedeckt werden. Ein Gleichgewicht ergibt sich dann, wenn die Grenzerträge des politischen Einflusses gleich den Grenzkosten der Einflußnahme sind (siehe Zeichnung).

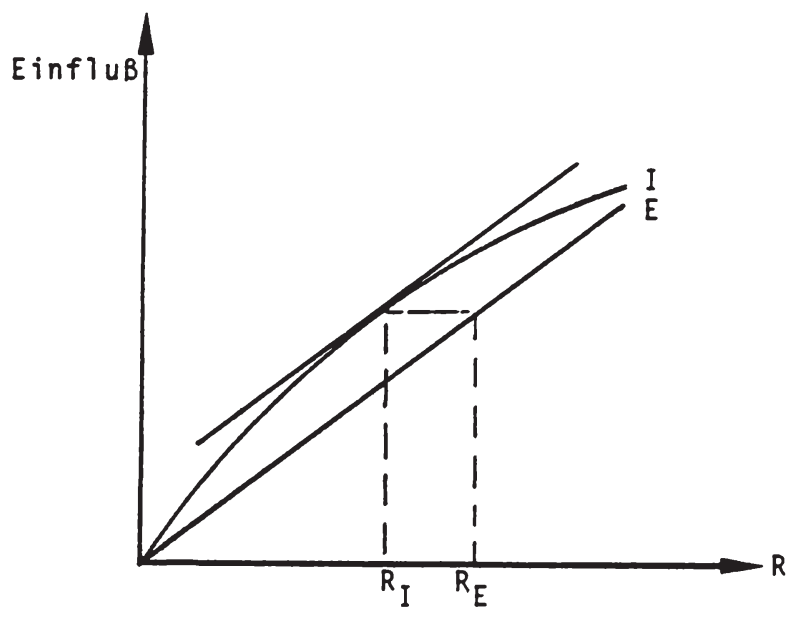

i) vgl. MCCORMICK/TOLLISON (1981) Chapt. 3; MCCORMICK/TOLLISON (1980) 
Auf der Ordinaten ist der $E$ influB, den eine Interessengruppe auf den politischen ProzeB hat, abgetragen, auf der Abszisse die in diesen investierten bzw. daraus gewonnenen Ressourcen R. I bezeichne nun eine Art "Produktionsfunktion des politischen Einflusses" in Abhängigkeit vom Ressourceneinsatz, also den Kosten der Einflußnahme. E kann entsprechend als Ertragsfunktion der EinfluBnahme interpretiert werden: Entlang $E$ läBt sich bestimmen, wie hoch der $E$ influB sein muB, um einen bestimmten Umverteilungsgewinn aus dem politischen ProzeB zu ziehen. Der Gewinn der Interessengruppe ergibt sich dabei als NettogröBe der gesamten Einkünfte aus dem politischen ProzeB und der darin investierten Ressourcen. Im betrachteten Fall gilt:

$$
G=R_{E}-R_{I}
$$

Die Kosten sind dabei von den Mitgliedern der jeweiligen organisation zu tragen und können aus verschiedenen Bestandteilen bestehen. Zu nennen sind:

a) die reinen Organisationskosten, d.h. Kosten, die durch die Notwendigkeit, die Interessen zu organisieren, entstehen,

b) Informationskosten: Es geht um das Sammeln und Auswerten von Informationen über die möglichen politischen Entscheidungen und deren Auswirkungen auf die Position der Mitglieder der Interessengruppen,

c) Propagandakosten: Hier geht es um die Verbreitung von Informationen uber die Auswirkungen von politischen Programmen und die Vorstellungen der Interessengruppe, um furr oder gegen bestimmte Entscheidungen Stellung zu nehmen bzw. Stimmung zu machen. Diese Informationskosten beinhalten auch die Weitergabe von möglichen Reaktionen der Interessengruppe auf bestimmte politische MaBnahmen an die Politiker. 
d) andere Arten von Ausgaben zur EinfluBnahme: Bestechungsgelder, 1) Wahlkampfspenden usw.

Dabei können c) und d) als direkte Einflußnahmekosten angesehen werden, während a) und b) eher vorgelagerte Kosten sind, ohne die jedoch auch eine Einflußnahme nicht möglich ist.

Letztendich führt die Existenz dieser "EinfluBnahmekosten" dazu, daB allein zur Herbeiführung von bestimmten Umverteilungsmaßnahmen Ressourcen eingesetzt werden, die anderweitig eine produktive Verwendung hätten finden können: Umverteilung wird zu einem Negativ-Summen-Spiel (TULLOCK, 1980), d.h. die Kosten von Umverteilungsmaßnahmen übersteigen die "tatsächlich" durch den Prozeß der Umverteilung selbst hervorgerufenen (allokativen) Kosten.

TULLOCK (1971) hat diesen ProzeB beschrieben: Ressourcen, die allein eingesetzt werden, um bestimmte Umverteilungsgewinne aus dem politischen Prozeß zu erzielen, sind vom individuellen Standpunkt aus gesehen $z$ war produktiv, aus gesellschaftlicher Sicht jedoch Verschwendung. Dabei setzt diese Verschwendung von Ressourcen nicht unbedingt voraus, daß es zu tatsächlichen Umverteilungsgewinnen für die jeweiligen Interessengruppen als Investoren kommt. Allein der Umstand, daß durch politische Einflußnahme Gewinne auf Kosten anderer Gruppen möglich sind, führt dazu, daB die einzelnen Interessengruppen zu einer Teilnahme am politischen ProzeB quasi gezwungen sind: wenn nicht, um eigene Gewinne zu erzielen, so doch zumindest, um zu verhindern, daB andere Gruppen Einkommensvorteile auf Kosten der betrachteten Interessengruppe durchsetzen. Nicht nur der Einsatz von Ressourcen, um Umverteilungsgewinne zu

i) vgl ROSE-ACKERMANN (1975) (1978), K. SCHMIDT (1969) (1978) 
erzielen, sondern auch um Umverteilungsverluste zu vermeiden, stellt eine gesellschaftliche Verschwendung dar.

Die Möglichkeit zur Einflußnahme auf politische Entscheidungen im Rahmen eines Nicht-Wahl-Mechanismus führt somit zu einem wie oben definierten "prozessualen Staatsversagen": Der Verlust an Ressourcen, die zur Entscheidungsfindung eingesetzt werden, ist höher als unter der "reinen" Majoritätsregel ohne Einflußnahme durch politische Interessengruppen - es kommt zu einem kostenverursachenden Wettbewerb um EinfluBnahme auf den politischen ProzeB, dessen $Z$ iel reine Umverteilungsmaßnahmen sind. Allerdings müssen nicht alle in die EinfluBnahme eingesetzten Ressourcen unbedingt als Verschwendung angesehen werden. Teilweise, z.B. bei der Bezahlung von Bestechungs geldern, dürfte es sich um reine Umverteilungsmaßnahmen zugunsten der Bestochenen handeln, die keinen allokativen Verlust darstellen und gegebenenfalls verteilungspolitisch sogar als positiv zu werten sind.1) Allerdings weicht dann die Einkommensverteilung von der im eigentlichen Wahlprozeß gewünschten ab.

Auch die Kosten der Organisation und die Kosten des Sammelns von Informationen sind nicht alle als Verschwendung anzusehen. Interessengruppen können durchaus in der Lage sein, Informationen billiger zu beschaffen, als es deren Mitglieder ohne diese Organisation könnten. In diesem Sinn wäre eine Situation ohne organisation noch weniger effizient als eine situation mit organisierter Interessenvertretung.

i) Vgl. SCHMIDT (1969) 
Auch kann die Verbreitung von Informationen unter Wähler und Politiker durchaus nur eine Verlagerung von Kosten, die sonst bei diesen angefallen wären, auf die Organisation bedeuten. Allerdings zeigen sich hier sehr starke Argumente fur eine Ineffizienz dieser Informationspolitik. Sinn der Bereitstellung der Information durch die Interessengruppe ist es ja, speziell die Argumente (Informationen) zu verbreiten, die für eine politische Entscheidung sprechen, die den von den Interessengruppen vertretenen Individuen Vorteile bringen, und den Zugang zu Informationen mit gegenteiligem Inhalt zu erschweren.

Ausgedrückt werden kann dies als Versuch, den Erwerb bestimmter Informationen zu verbilligen und diese damit leichter zugänglich zu machen, bzw. andere zu verteuern.1)

Betrachtet werden soll das Informationskalkïl des Wählers. Dabei soll davon ausgegangen werden, daß die subjektiven (erwarteten) Kosten auch den tatsächlichen Kosten, die mit der Möglichkeit einer falschen Entscheidung aufgrund eines unzureichenden Informationsstandes verbunden sind, entsprechen, d.h. daß die Erwartungen der Individuen richtig sind. Dann ergibt sich die optimale Informationsmenge für den Wähler an dem Punkt, an dem die Grenzkosten der Informationsbeschaffung gleich den Grenzerträgen der besseren Informiertheit bzw. den vermiedenen Grenzkosten einer auf mangelnden Informationen beruhenden falschen Entscheidung sind. Diese optimale Informationsmenge fuihrt zu einer Minimierung der Kosten der Uninformiertheit. Einflußnahme wird dadurch möglich, daß man das Kostenkalkül der Wähler verändert. Wenn die Interessengruppe an einer positiven Entscheidung interessiert ist, wird sie die Informationen, die für diese Entscheidung sprechen,

1) vgl. zum folgenden BARTLETT (1973), S. $31 \mathrm{ff.}$, S. $130 \mathrm{ff}$. 
verbilligt zur Verfügung stellen. Beispiel dafür sind Anzeigenkampagnen, kostenlose Abgabe von Informationsbroschüren, Flugblattaktionen usw.

Dieser Zusammenhang ist in der folgenden Zeichnung dargestellt. Die für den Wähler relevanten Grenzkosten der Informationsbeschaffung sinken dabei auf $\mathrm{C}^{\prime} 2$, der Wähler wird insgesamt mehr (spezifische) Informationen nachfragen als im Fall ohne Subventionierung. Die vom Wähler zu tragenden Informationskosten werden be $I^{* \star} k l e i n e r$ sein als bei I* - die tatsächlichen Kosten der Informiertheit liegen jedoch höher, nämlich um die Differenz zwischen den tatsächlichen Kosten der Information $c * \star$ und den Minimalkosten der Informiertheit $C^{*}$. Der Wähler ist auf diesem Gebiet "uberinformiert".

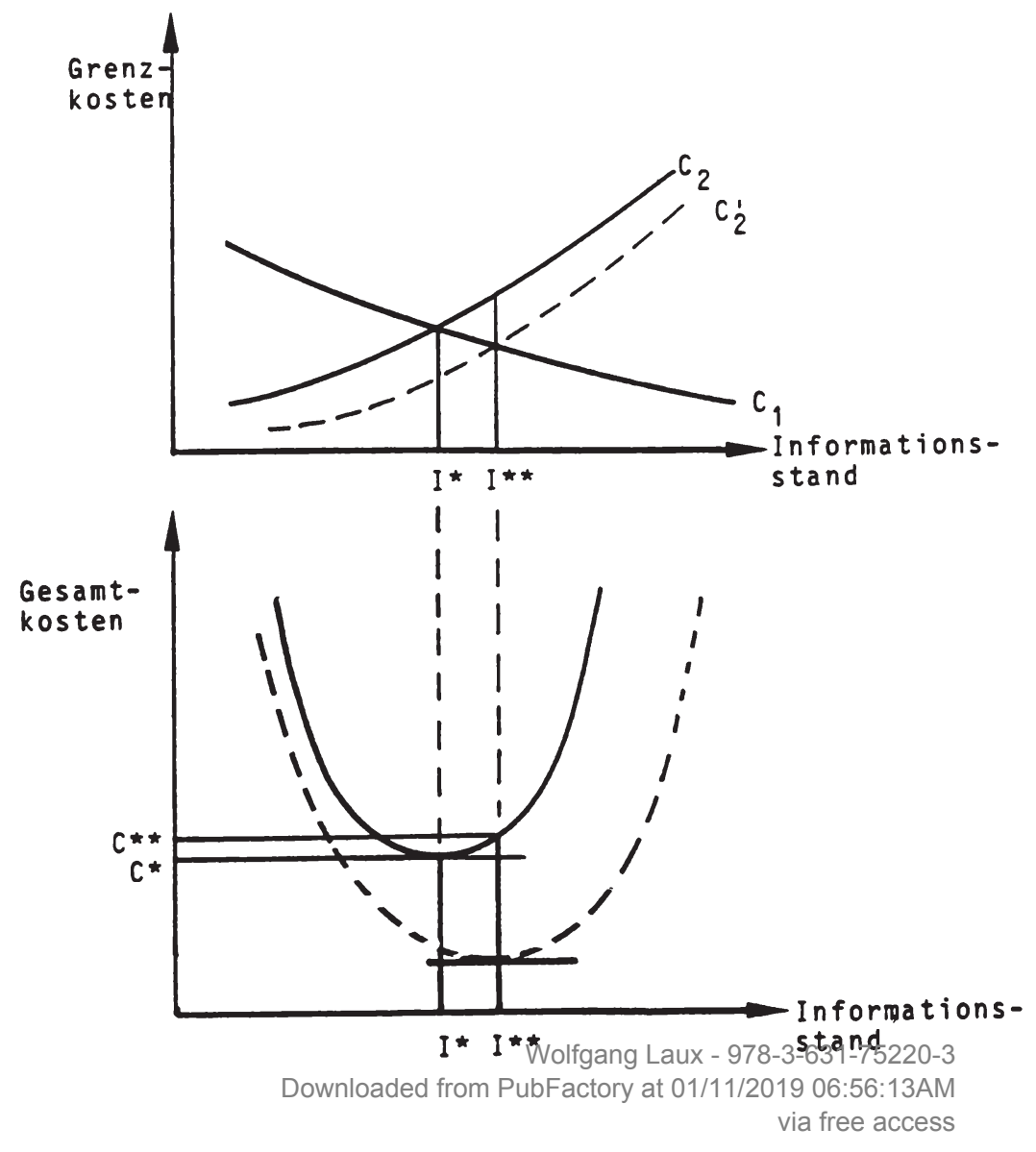


Umgekehrt werden Argumente, die gegen eine Maßnahme sprechen, deren Durchsetzung einer Interessengruppe gelegen kommt, zuückgehalten bzw. die Information über die Nachteile dieser Maßnahme verteuert. In diesem Fall wird der Wähler sich entscheiden, weniger an Informationen nachzufragen, also in einem geringeren Maße informiert sein als - objektiv gesehen - optimal ist. Auch hier liegen die Kosten $C \star \star$ des - diesmal zu geringen -Informationsstandes über denen der Minimalkostenkombination $C^{\star}$.

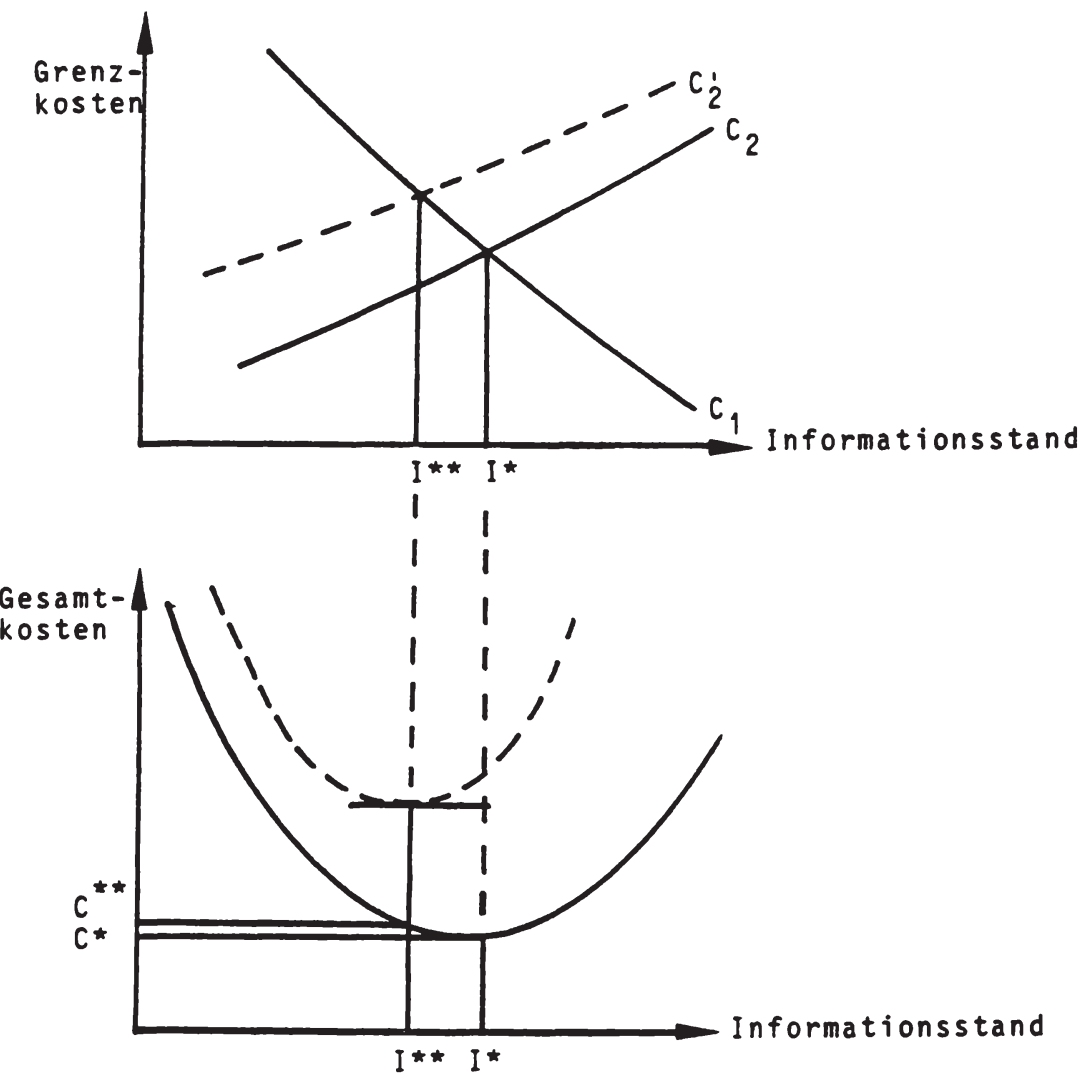


Die hier für die Wähler abgeleiteten Zusammenhänge gelten natuirlich auch für die Politiker und ihre Informationsbedürfnisse. Auch sie müssen sich über die Auswirkungen von Maßnahmen auf die möglichen Stimmengewinne und -verluste Gedanken machen. Auch hier können die Interessengruppen EinfluB nehmen, indem sie verzerrte Angaben über die zu erwartenden Wirkungen einer bestimmten politischen Entscheidung machen. Der Mechanismus ist dabei im wesentlichen derselbe wie im eben dargestellten Fall der Beeinflussung der Wähler, und führt ebenfalls zu einem Informationsstand, der anders ist als der mit Minimalkosten verbundene.

3.3.4.2 Ein Gleichgewichtsmodell des politischen Einflusses

Im folgenden soll ein Gleichgewichtsmodell für die Höhe der politischen EinfluBriahme dargestellt werden, um die Ergebnisse des vorhergehenden Abschnitts noch einmal zu verdeutlichen.1)

Dabei soll von einem Modell mit zwei Interessengruppen ausgegangen werden, die um politischen EinfluB zur Erzielung von Umverteilungsgewinnen konkurrieren. Dabei ist davon auszugehen, $d a B$ die Höhe der Einflußnahmekosten, die eine Interessengruppe auf sich nehmen muB, um einen bestimmten Umverteilungsgewinn zu realisieren, nicht unabhängig ist vom Einsatz von Ressourcen, die die andere Interessengruppe in den politischen ProzeB investiert. Die Begründung liegt auf der Hand: Jeder Umverteilungsgewinn, den die andere Gruppe macht, führt zu einer verringerten Chance, selbst Gewinne zu erzielen, bzw. zu einer direkten

\footnotetext{
i) Zum Modellaufbau vgl. BECKER (1983)
} 
Belastung über Steuerzahlungen, die den Umverteilungsgewinn aus der Beteiligung am politischen Prozeß verringern.

Für die einzelnen Interessengruppen gilt deshalb:

$$
\begin{aligned}
& \operatorname{Tr}_{i}=\operatorname{Tr} r_{i}\left(R_{i}, R_{j}\right) \\
& \operatorname{mit} \frac{\partial T r_{i}}{\partial R_{i}}>0, \frac{\partial T r_{i}}{\partial R_{j}}<0 \\
& \frac{\partial^{2} T r_{i}}{\partial R_{i}^{2}}<0, \frac{\partial^{2} T r_{i}}{\partial R_{j}^{2}}>0 \\
& \frac{\partial^{2} T_{i}}{\partial R_{i} \partial R_{j}} \leqslant 0
\end{aligned}
$$

mit $\operatorname{Tr}_{j} \quad$ = Umverteilungsgewinn (Transfer) der Gruppe $i$

$R_{i, j}=$ von Gruppe $i, j$ in den politischen Prozeß eingesetzte Ressourcen

Im einzelnen bedeutet das, daß die Höhe der Transfers negativ abhängig sind von der Menge an Ressourcen, die die andere Interessengruppe in den politischen Prozeß investiert. Es ist möglich, daß diese Transfers auch negativ werden können, d.h. daß durch die Aktivität anderer Interessengruppen eine Gruppe i zu Nettozahlern wird.

Des weiteren wird angenommen, $d a ß$ die beiden Interessengruppen die Gesamtheit der in der Gesellschaft vorhandenen Individuen umfassen. Es gilt somit folgende Budgetbeschränkung:

$$
\sum_{k=i, j} T r_{k}=0
$$


d.h. positive Umverteilungsgewinne, die die eine Gruppe im politischen ProzeB erzielt, werden von der anderen Gruppe bezahlt. (Der Einfachheit halber wird hier davon ausgegangen, daB der Wert der empfangenen und entnommenen Leistung einander entsprechen.)

Dieser Zusammenhang läBt sich für die Interessengruppe $i$ unter der Annahme

$\frac{\partial^{2} T_{i}}{\partial R_{j} \partial R_{j}}>0$

folgendermaßen graphisch darstellen:

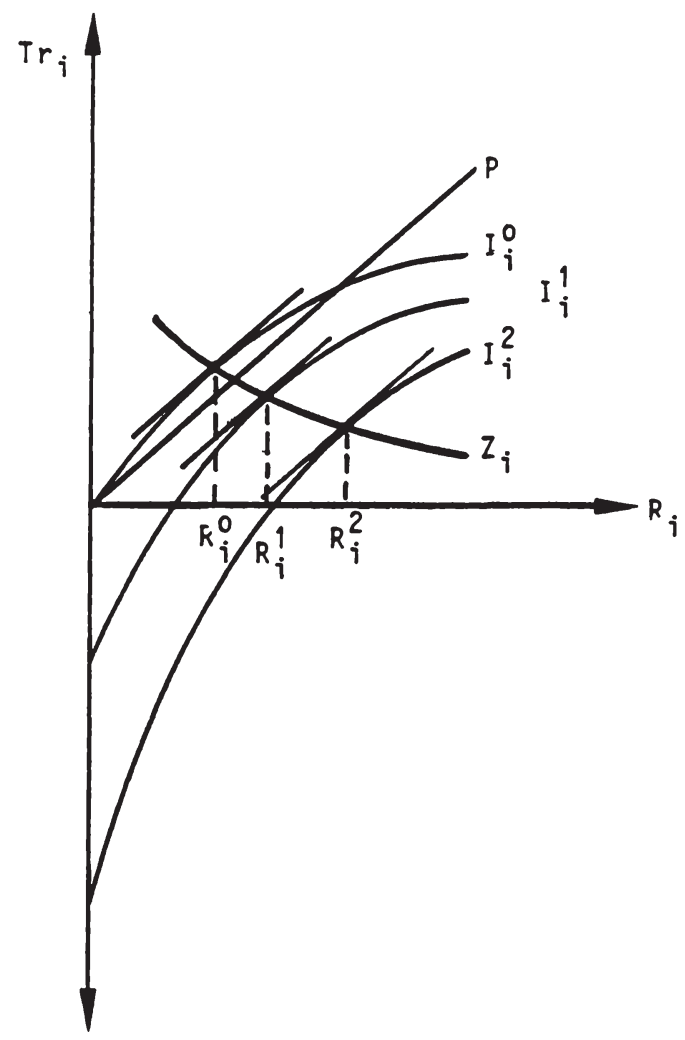

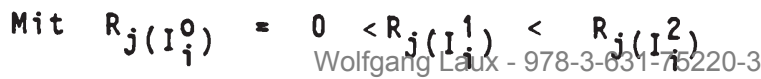


Auf der Ordinaten wird die Höhe der Umverteilungsgewinne (-verluste) abgetragen, auf der Abszisse die Menge an Ressourcen, die in den politischen EinfluBnahmeprozeB investiert wurden. Die Steigung der Geraden $P$ bezeichnet die (hier aus Vereinfachungsgründen konstant gehaltene) Substitutionsrate von öffentlichen Transfers und privaten Ressourcen, also quasi deren relatives Preisverhältnis.

Die verschiedenen I-Kurven zeigen an, welche Umverteilungsgewinne (oder -verluste) die Interessengruppe $i$ durch den Einsatz von Ressourcen in den politischen ProzeB machen kann bei jeweils gegebener Einflußnahme bzw. Höhe des Ressourceneinsatzes der Interessengruppe $j$. Ein optimum für die Interessengruppe besteht immer dann, wenn die Grenzerträge des Einsatzes von privaten Ressourcen in den politischen ProzeB $\partial \mathrm{Tr}_{j} / \partial R_{j}$ gleich sind der Grenzrate der Substitution von öffentlichen Transfers und privaten Gütern, die durch die Steigung der $P-G e r a d e n$ gegeben ist. Dabei ergibt sich ein Minimum an eigenem Ressourceneinsatz (und ein Maximum an Transfergewinnen) für Gruppe $i$, wenn Gruppe $j$ keine Ressourcen in den politischen ProzeB einsetzt (Kurve $I^{i} 0$ ). Bei zunehmender EinfluBnahme durch Gruppe $j$ steigt auch die von Gruppe $i$ in den politischen ProzeB eingesetzte Ressourcenmenge, wobei die erzielten Erträge auch negativ sein können, d.h. die Interessengruppe zum Nettozahler wird. Aus $\Sigma T r_{k}=0$ folgt, daß umgekehrt die Grenzproduktivität der von der Interessengruppe $j$ in die politische Einflußnahme eingesetzten Ressourcen sinkt, wenn $R_{j}$ steigt. Für die Interessengruppe $j$ folgt ein mit zunehmendem $R_{i}$ abnehmender Einsatz von eigenen Ressourcen, es gilt:

$\frac{\partial^{2} T r_{j}}{\partial R_{i} \partial R_{j}}<0$

Ein Gleichgewicht läBt sich jetzt folgendermaßen ableiten: $Z_{i}$ bezeichne eine Anpassungskurve, die die Reaktion der Interessengruppe $i$ auf eine Erhöhung der von $j$ in den 
politischen ProzeB eingesetzten Ressourcen darstellt. Geht man von einem Verhalten unabhängiger Anpassung aus, d.h. die jeweils betrachtete Gruppe nimmt die Höhe der Einflußnahme der anderen Gruppe als gegeben an und paßt ihren eigenen Ressourceneinsatz in den politischen ProzeB an, ohne mögliche Wirkungen auf das Verhalten der anderen Gruppe zu bericksichtigen, dann läBt sich ein Gleichgewichtspunkt für den Ressourceneinsatz in den politischen ProzeB konstruieren. Dieser ergibt sich als Schnittpunkt der Anpassungskurven $Z_{i}$ und $Z_{j}$.

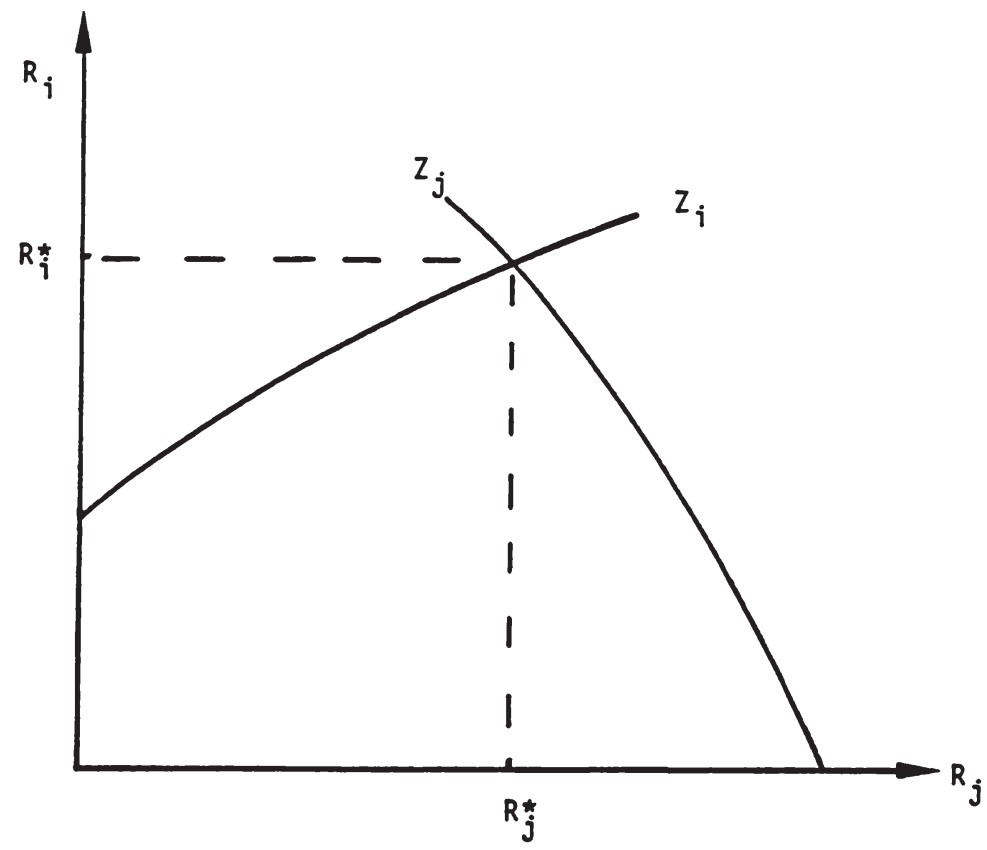


Voraussetzung für ein Gleichgewicht ist, daß die Steigung der $Z_{j}$-Kurve $k$ leiner ist als die Steigung der $Z_{j}$-Kurve.1)

Das Zwei-Interessengruppenmodell verdeutlicht noch einmal den Umstand des prozeßbezogenen Staatsversagens: Beide Gruppen setzen Ressourcen in Höhe von $R_{j}$ * $b z w . R_{j}$ in den politischen ProzeB ein, die verloren sind.

\subsubsection{Situationsbezogenes Staatsversagen und politischer} EinfluB

Im Rahmen der Interessengruppentheorie des Staates läBt sich allerdings nicht klären, ob eine ungleiche Verteilung des Einflusses auch zu einer ineffizienten Allokation führen muB. Eine ungleiche Machtverteilung führt im wesentlichen dazu, daß die mit höherer Macht ausgestatteten Individuen ein positives Einkommen durch den politischen Prozeß erzielen können, also Umverteilungsgewinne realisieren. Ober die spezifische Art dieser Umverteilungsgewinne ist damit noch nichts gesagt.

Ein Beispiel eines Interessenverbandes mag in diesem Zusammenhang ein Verband von Baunternehmern sein. Nach der herkömmlichen Theorie der Einflußgruppen könnten diese darauf drängen, mehr an öffentlichen Gebäuden durch statliche Mittel herzustellen, und dabei z.B. auf die Wählerstimmen der in der Bauindustrie tätigen Arbeiter verweisen. Unter diesen Umständen kommt es gegebenenfalls $z u$ einer zu großen Versorgung mit Gebäuden, d.h. die Ausgaben für öffentliche Bauten überschreiten das optimale, von den Wählern an sich gewünschte Maß.2) Die

1) Dazu BECKER (1983)

2) Man könnte diese Situation auch beschreiben als einen im politischen ProzeB sich manifestierenden Nutzenvergleich. Tatsächlich wird die Erhöhung der Einkommen der Mitglieder der Lobby stärker bewertet als die 
Voraussetzung für ein erfolgreiches Operieren dieser Lobby seien erfültt - hohe gruppeninterne Erträge, geringe Organisationskosten, begrenzte Gruppengröße usw.

All dies als gegeben, stellt sich die Frage, weshalb die Lobbyisten den (kostspieligen) Weg der Erhöhung der für die Herstellung von Gebäuden verwandten Ausgaben wählen. Der Sinn dieser Maßnahme liegt ja weniger darin, daß eine Erhöhung der Ausgaben an sich für wünschenswert gehalten wird, sondern es handelt sich um einen Versuch, über diese Ausgaben höhere Einkommen (Gewinne) zu erzielen. Wenn die Gruppe der Bevorteilten bekannt ist, bleibt zu fragen, weshalb diese ihren Einfluß nicht darauf richten, direkte Einkommenstransfers zu erhalten, sondern stattdessen den komplizierten Umweg über erhöhte Aufträge wählen. Die Belastung der Steuerzahler bliebe die gleiche, der Gewinn fur die Mitglieder oder die Klientel des Interessenverbandes wäre höher, und es bestände keine Gefahr, daß mögliche Gewinne durch das Hinzutreten und die Aktivität neuer Produzenten auf diesem Sektor wegkonkurriert wiurden. 3 ) Wenn aufgrund eines besonders starken Einflusses eine Erhöhung des eigenen Einkommens via politische

Verschlechterung der Einkommensposition derjenigen, die über Steuern die Erhöhung der Bauausgaben finanzieren.

3) Diese Gefahr besteht immer dann, wenn die Abgabe der öffentlichen Leistungen nicht an bestimmte Personen gebunden ist, sondern spezifische Kriterien zur Vorausssetzung hat, die im Moment des Beschlusses von den Mitgliedern der betreffenden Interessengruppe erfüllt wurden. Als Beispiel sei die Subventionierung von Milchprodukten genannt, die von allen Milchproduzenten gleichermaßen in Anspruch genommen werden kann. Sind dann durch die Milchproduktion höhere Gewinne zu erzielen als durch andere Produkte, werden auch andere Produzenten in diesen Sektor vorstoßen und damit die Voraussetzung der Subventionsvergabe erfüllen. Sinkende Preise aufgrund erhöhter Produktionsmengen werden dann die Gewinne (auch der ursprünglichen Lobbyisten) auf ein Normalmaß reduzieren. Zur Konkurrenz im RentSeeking-Modell vgl. POSNER (1975), TULLOCK (1975) 
Entscheidung möglich ist, dann ist es in einem Höchstmaße irrational, diesen wenig effizienten weg zu wählen. Geringe Effizienz ist aber immer dann der Fall, wenn nicht-freie Transfers als Ergebnis des politischen Prozesses herauskommen.1)

Hinzu kommt noch, daß für jede Interessengruppe ein Anreiz besteht, möglichst effiziente Methoden der Realisierung von Umverteilungsgewinnen zu wählen. Je größer die auf den eigentlichen Verteilungsgewinn bezogene Ineffizienz ist, d.h. je mehr Mittel über das Budget bewegt werden mussen, um einen bestimmten Verteilungsgewinn zu realisieren, um so höher wird der Widerstand anderer Gruppen sein und um so höhere Einflußnahmekosten entstehen den Mitgliedern der betrachteten Interessengruppe. Aus diesem Grund ergibt sich für jede Interessengruppe ein Anreiz, möglichst effiziente Wege, d.h. mit möglichst geringen Zusatzkosten belastete Methoden der Realisierung von Umverteilungsgewinnen zu wählen. 2)

Wenn aber die Interessengruppen ihren $E$ influß dahingehend geltend machen können, daß sie bestimmte freie Transferzahlungen erhalten, handelt es sich um reine Umverteilungsmaßnahmen, uber deren Wuinschbarkeit objektiv kaum eine Aussage zu machen ist. Ein Staatsversagen kann es in diesem Sinne nicht geben, höchstens eine Abweichung von dem Endzustand, der sich bei einer reinen Mehrheitsentscheidung ohne Einflußnahme der Interessengruppen ergeben würde.

1) Dasselbe Phänomen ist auch in den meisten Studien der Public-Choice-Literatur zu finden, soweit die Bereitstellung öffentlicher Güter mit Verteilungsfragen verknüpft ist. Es wird selten erklärt, warum diese Umverteilung ausgerechnet über die Versorgung mit öffentlichen Gütern stattfindet, und nicht über direkte Transfers.

2) BECKER (1983) 
Der Grund, daß die suboptimale Lösung gewählt wird, liegt im Informationsgehalt solcher Maßnahmen.

Ein Spielraum für die politische Einflußnahme ist nur solange gegeben, wie eine mögliche Bevorteilung bestimmter Gruppen nicht $z u$ entsprechenden Gegenmaßnahmen der negativ tangierten Gruppe führt. Solche Gegenmaßnahmen können zum Beispiel darin bestehen, daß die negativ Betroffenen sich selbst organisieren oder $d a B$ sie anderen Parteien ihre Stimme geben und somit den Wahlerfolg der die spezifischen Gruppen begünstigenden Politiker in Frage stellen. $O b$ es dazu kommt, hängt aber vom Informationsstand der Betroffenen ab. Dabei wird aber die Höhe der Kosten, die bei der Beschaffung von Informationen über die Auswirkungen und verteilungspolitischen Inhalte einer Maßnahme anfallen, wesentich von deren spezifischer Ausgestaltung bestimmt. Eine reine Umverteilung über Barzahlungen ist leicht als solche $z u$ identifizieren und wird damit zu Stimmverlusten bei allen von der Umverteilung negativ Betroffenen führen. Die Durchsetzung einer solchen offenen Umverteilungsmaßnahme düfte also kaum möglich sein, der diskretionäre Spielraum für offene, ungebundene Transfers zugunsten rein personell abgegrenzter $k$ leiner Gruppen duifte entsprechend gering sein.1)

Höhere Informationskosten entstehen dann, wenn die Umverteilung nicht explizit, sondern implizit uiber bestimmte Ausgabenkategorien (Staatsaufträge, Subventionen usw.) erfolgt. Für die Steuerzahler ist dann nicht mehr eindeutig sichtbar, ob eine solche Maßnahme rein gruppenegoistischen Interessen dient oder auch positive Auswirkungen auf die eigene Lage hat. Die Problematik der

1) $\mathrm{vgl}$ NELSON (1976) S. 323, TULLOCK (1967) S. $103 \mathrm{ff}$. Ähnlich argumentiert RUBIN (1975). Dieser führt eine Bevorzugung ineffizienter Transferarten darauf zurück, daß diese in größeren zeitlichen Abständen iberprüft würden als (effiziente) Geldleistungen. 
Kenntnis der Auswirkungen öffentlicher Maßnahmen auf die eigene Position wurde ja bereits angesprochen. Damit entstehen für die einzelnen Steuerzahler hohe Informationskosten, die sie aufwenden müßten, um die Maßnahme zu evaluieren. Die Existenz solcher Informationskosten ist aber notwendig, damit einzelne Interessengruppen Vorteile gegenüber anderen Gruppen durchsetzen können. Daher besteht für die jeweilige Interessengruppe ein Anreiz, die Undurchschaubarkeit des Budgets zu erhöhen und damit partiell die Wirksamkeit der politischen Konkurrenz um Wählerstimmen über Budgetvorschläge außer Kraft zu setzen.

Das Kalkül der Interessengruppen kann dann berechnet werden:

$$
\text { Max: } U=U(\operatorname{Tr}, R) \text { mit } \operatorname{Tr}=\operatorname{Tr}(B, F)
$$

Wobei Tr den Einkommenswert oder Verteilungsgewinn aus einer spezifischen statichen Leistung symbolisiert. Dieser ist abhängig von den für die staatliche Leistungen aufgewendeten realen Ressourcen $B$ (also dem Umfang des Budgetpostens), und dem Wert $F$ als Ausdruck für den Grad der Ineffizienz dieser staatiichen Umverteilungsmaßname, die durch die spezifische Art der Umverteilung gegeben ist.

Für die Höhe der bei diesem UmverteilungsprozeB aufgewandten Ressourcen $B$ gelte:

$$
B=B(R, W)
$$

Wobei $R$ die durch die Interessengruppen involvierten Kosten darstellen (Mitgliedsbeiträge, Lobbykosten, Bestechungsgelder usw.), die den einzelnen Mitgliedern der 
Interessengruppen entstehen. W gilt als Indikator des Widerstands anderer Gruppen bzw. der Wähler insgesamt (durch Gegenstimmen usw.).

Es gelte: $\frac{\partial T r}{\partial B}>0, \frac{\partial T r}{\partial F}<0, \frac{\partial 2 T r}{\partial B \partial F}<0$

Damit ergibt sich fü die Zielfunktion der Interessengruppe:

$$
\text { Max: } U=U\{\operatorname{Tr}(B(R, W), F), R\}
$$

Wobei Handlungsparameter die für die Erlangung der Transfers eingesetzten Ressourcen $R$ und der Grad der Ineffizienz $F$ der staatlichen Maßnahme sind.

Es gilt: $\frac{d U}{d R}=\frac{\partial U}{\partial T r} \frac{\partial T r}{\partial B} \frac{\partial B}{\partial R}+\frac{\partial U}{\partial R}=0$

Aus: $\quad \frac{\partial U}{\partial T r}=\frac{-\partial U}{\partial R}$ folgt: $\frac{\partial T r}{\partial B}=\frac{\partial R}{\partial B}$

Die einzelnen Mitglieder der Interessengruppen erreichen dann ihr Nutzenmaximum, wenn die Verteilungsgewinne aus einer Erhöhung des staatlichen Budgets gleich sind dem Wert der Ressourcen, die sie für diese marginale Erhöhung ausgeben müssen.

Differenzieren wir nach $F, g i l t$ :

$\frac{d U}{d F}=\frac{\partial U}{\partial T r} \quad \frac{\partial T r}{\partial F}=0$;

$D a \frac{\partial T r}{\partial F}<0$ für $F>0$ muß $F=0$ sein.

Die Situation ändert sich, wenn die Widerstandsfunktion der anderen Bürger nicht unabhängig ist vom eigenen Verhalten. Gesetzt den Fall, es gilt: $\partial W / \partial F<0, d . h$. die möglichen entstehenden Widerstände durch zu erwartende 
Stimmverluste hängen ab von der Durchsichtigkeit der politischen Maßnahme, die negativ mit $F$ (als waste-Indikator) korreliert.

Es folgt dann:

$$
\begin{aligned}
\text { Max !: } U= & U \\
\text { mit } & (T r, T) \\
& \operatorname{Tr}=\operatorname{Tr} \quad(W, F) \\
B & =B \quad(W, R) \\
W & =W \quad(F)
\end{aligned}
$$$$
\text { mit } \frac{\partial W}{\partial F}<0
$$

Es folgt:

$\frac{d U}{d F}=\frac{\partial U}{\partial T r} \frac{\partial T r}{\partial B}+\frac{\partial B}{\partial W} \frac{\partial W}{\partial F}+\frac{\partial U}{\partial T r} \frac{\partial T r}{\partial F}=0$

oder $\frac{\partial T r / \partial F}{\partial T r / \partial B}=-\frac{\partial B / \partial W}{\partial F / \partial W}<0$

Sinkt durch eine Erhöhung der Ineffizienz einer Maßnahme deren Informationsgehalt über die damit verbundenen Verteilungswirkungen, so verringert sich der Widerstand der Wähler. Die Interessengruppe kann daher ein höheres Budget durchsetzen. Andererseits sinkt aufgrund der erhöhten Ineffizienz der Anteil der Umverteilungsgewinne an den statlichen Gesamtausgaben. Beide Effekte wird die Interessengruppe gegeneinander abwägen und den Grad der Ineffizienz wählen, bei dem sich marginale Gewinne und Verluste ausgleichen.

\subsubsection{Die Behandlung des Fiskalillusion in der Litera- tur}

Die Oberlegung, daß die Entscheidung der Wahlbürger nicht von der Höhe der Kosten und Vorteile der Staatstätigkeit, sondern von der "wahrgenommenen" Höhe dieser Größen abhängt, ist nicht neu. Insbesondere bei Diskussionen um 
die optimale GröBe des Budgets wurde dieser Zusammenhang unter dem Begriff der "Fiskalillusion" behandelt. Dabei ist man sich über die Auswirkungen der Fiskalillusion nicht einig.

DOWNS (1960) argumentierte, daß die aktuelle Höhe des Budgets kleiner sei als die "korrekte" Größe, wobei diese definiert war als der Budgetumfang, der sich ergeben würde, wenn die Wähler vollkommen über Nutzen und Kosten der staatlichen Maßnahmen informiert wären. Zur Begrindung dieser These fuhrt DOWNS auf, daB die Wahrnehmung der Steuerbelastung stärker wäre als die Wahrnehmung des Nutzens, den der einzelne aus dem Statshaushalt zieht, und daß deshalb ein stimmenmaximierender Politiker ein zu kleines Budget anbieten wïrde.

Andere Beiträge stellen im Gegensatz zu DOWNS eher auf die Einnahmeseite $a b$ und argumentieren, $d a B$ "Fiskalillusion" hauptsächlich an dieser Stelle auftreten wïrde und es somit zu einem zu großen Umfang des Budgets käme.1)

Letztendlich läBt sich über die Auswirkung der Fiskalillusion auf die Optimalität des bestehenden Budgetumfangs nichts aussagen. Versuche, durch einen Vergleich der Höhe der Fiskalillusion auf der Einnahme-und Ausgabenseite (im Sinne einer Unterschätzung der Lasten bzw. Nutzen) herauszufinden, ob der Budgetumfang zu groB (Dominanz der Fiskalillusion auf der Einnahmeseite) oder zu klein (stärkere Ausgabenillusion) ist, dürften wenig hilfreich sein.2) Gibt man nämlich die Voraussetzung nur eines öffentlich angebotenen Gutes bzw. nur einer Einnahmeart auf, dann dirfte auch die Struktur der Ausgaben und

1) Zur Fiskalillusion auf der Einnahmeseite vgl. WAGNER (1976), GOETZ (1977), FLOWERS (1977), BROWNING (1974), POMMEREHNE/SCHNEIDER' (1978), SCHMOLDERS (1959)

2) vgl. WEST/WINER (1980), CARTER (1982) 
Einnahmen durch die Existenz der Fiskalillusion nicht unberührt bleiben. Bei gegebenem Budgetumfang können dann bestimmte Ausgaben- oder Einnahmenkategorien zu hoch, andere zu niedrig sein, der Gesamteffekt bleibt offen.

Bei den genannten Autoren handelt es sich bei der "Fiskalillusion" um ein nicht geklärtes, exogen gegebenes Phänomen. DaB die Verbreitung von Fiskalillusion ein Mittel sein kann, um bestimmte Verteilungswirkungen des Staatshaushalts systematisch zu verschleiern und damit den Widerstand der Steuerzahler zu minimieren, wurde bereits von dem Italiener PUVIANI um die Jahrhundertwende erkannt.1) PUVIANI ging dabei von einem durch eine herrschende Klasse dominierten Stat aus, die die Illusion benutzt und fördert, um eigene Privilegien zu erhalten und zu schützen. "Schlieblich erkennt man ein drittes friedliches Mittel des Schutzes gegen die Unzufriedenen in den optimistischen Illusionen betreffs der öffentlichen Einnahmen und Ausgaben. Mit ihrer Hilfe wird ein beträchtlicher Teil der Steueropfer der Bürger getarnt, der Glaube genährt, daB viele Institutionen und Formen öffentlicher Betätigung vorteilhaft seien, der Wert der vom State durchgeführten Reformen übertrieben. Man kann somit zu der Annahme kommen, daB die optimistische Illusion dem Stat eine größere Stabilität verleiht."2)

PUVIANI geht dabei weiterhin im einzelnen auf die verschiedenen Einnahmearten ein und schildert deren (vermutete) Wirkung auf das Belastungsgefühl der Statsbuirger, beschreibt aber auch die Möglichkeit zur Verschleierung von Ausgaben durch haushaltstechnische und andere Kniffe.

1) PUVIANI (1960). Das italienische Original erschien 1903. Eine detaillierte Inhaltsangabe findet man bei BUCHANAN (1967).

2) PUVIANI (1960) S. 25 
Die meisten Beiträge, die sich mit Fiskalillusion befassen, behandeln allerdings nicht die Schaffung von Fiskalillusion als ein Ergebnis eines rationalen Entscheidungsprozesses, wie es bei PUVIANI und BARTLETT begriffen wird, sondern begnügen sich, das Phänomen zu erfassen und daraus Politikempfehlungen abzuleiten.1)

Eine besondere Rolle bei der Diskussion der Fiskalillusion spielt dabei die in den meisten Staten zu beobachtende wachsende Statsverschuldung. Besonders BUCHANAN's und WAGNER's Kritik2) an den ökonomischen Folgen des Keynesianismus betont, daB die Möglichkeit der Staaten zur Verschuldung zu einem überhöhten Statshaushalt geführt habe.

Ihrer Meinung nach existierte vor der keynesianischen Revolution als ein Teil der Finanzverfassung die Vorstellung eines jährlichen, materiellen Budgetausgleichs, d.h. ein Verbot der Schuldfinanzierung von öffentichen Ausgaben. Wenn auch eine solche Vorschrift nicht explizit in den Verfassungen zum Ausdruck $\mathrm{kam}$, so bestand doch ein allgemeiner Konsens, der einer Kreditfinanzierung von Staatsausgaben in Normalzeiten (Kriege galten als Ausnahmezeiten, eine Kriegsfinanzierung durch Schuldaufnahme wurde allgemein akzeptiert) den Ruch der Unsolidität anhängte und somit quasi als eine implizite Regel wirkte, die diese Art der Finanzierungsweise weitestgehend ausschaltete.

1) Z.B. WAGNER (1976), MUSGRAVE (1981), die beide eine Vereinfachung des Steuersystems postulieren, bzw. durch die Integration von Einnahme- und Ausgabeentscheidungen eine höhere Transparenz der Entscheidungen schaffen wollen, oder WEST/WINER (1980), die einen optimalen Grad der Fiskalillusion ableiten.

2) BUCHANAN/WAGNER (1977) (1978a) (1978b) 
Die keynesianische Revolution veränderte die Einstellung gegenuber der Staatsverschuldung als Finanzierungsinstrument, indem das Augenmerk auf die stabilisierenden Effekte des Staatshaushalts gelenkt und die staatliche Kreditaufnahme als geeignetes Mittel zur Bekämpfung der Rezession dargestellt wurde.

Die Enttabuisierung der Staatsverschuldung zog jedoch eine übermäBige Ausdehnung der Staatsausgaben nach sich: Gegenüber einer Erhöhung von Steuern bzw. einer Reduktion von Ausgaben stellt die Kreditaufnahme eine Art des Ressourcenentzugs dar, die ein relativ geringes Belastungsgefühl auf Seiten der Statsbürger hinterläBt bzw. deren negativen Auswirkungen für den einzelnen Bürger schwer feststellbar und quantifizierbar sind. Daraus resultiert eine Unterschätzung der tatsächlichen Belastung durch die Staatsverschuldung, mit dem Effekt, daB es zu einer zu großen Ausdehnung der Staatsausgaben kommt.

Der Grund liegt nach BUCHANAN/WAGNER in einem unsymmetrischen Einsatz des keynesianischen Instrumentariums: In der Rezession werden Steuerausfälle und konjunkturpolitisch begrüdete Mehrausgaben durch Schuldaufnahme finanziert, im Boom kommt es, anders als von der keynesianischen Doktrin vorgesehen, jedoch nicht zu einer Bildung von Oberschüssen bzw. zu einer Reduktion des Schuldenbestandes, da die Leidtragenden solcher Maßnahmen relativ leicht $z u$ identifizieren sind und damit dadurch starke politische widerstände hervorgerufen werden, die einen stimmenmaximierenden Politiker veranlassen, darauf zu verzichten und weiterhin zur kreditaufnahme zu greifen. 
BUCHANAN/WAGNER fordern entsprechend die Aufnahme eines Artikels in die Verfassung, der einen jährlich materiell ausgeglichenen Haushalt zur Vorschrift macht, und die Statsverschuldung als Finanzierungsinstrument somit ausschließt.

Die Forderung nach einer Reduktion der Statsverschuldung findet, wenn auch oft aus anderen Gründen, eine groBe Anzahl von Befürwortern. Die Idee einer verfassungsmäBigen Begrenzung der Schuldaufnahmemöglichkeit, die ja auch einer Initiative einer Anzahl von Bundesstaaten in den USA zugrunde $\mathrm{lag}, 1$ ) wäre auch im Rahmen einer Arbeit über den Sinn von Budgetbeschränkungen eingehender $z u$ behandeln. Allerdings liegen zum Problem eines verfassungmäBig verankerten $Z$ wangs zum materiellen Haushaltsausgleich bereits einschlägige Arbeiten vor, so daB sich eine tiefere Behandlung hier erübrigt.2)

\subsubsection{Exkurs: Bürokratie als Interessengruppe}

Einer besonderen Berücksichtigung unter den verschiedenen Interessengruppen bedarf die Bürokratie. In Abschnitt 3.2 wurde gezeigt, daß eigennutzorientierte Bürokratien an einem möglichst hohen Budget interessiert sind. Dabei wurde aber das Verhalten der Bürokratie nur insoweit in Betracht gezogen, wie diese als Anbieter öffentlicher Leistungen fungieren, während die Nachfrage nach öffentlichen Leistungen eine exogen gegebene Größe darstellte.

1) siehe dazu Kapitel 1.

2) vor allem: BAUM (1982) und die erwähnten Schriften von BUCHANAN / WAGNER. 
$Z u$ beachten ist allerdings, $d a B$ die Nachfrage nach öf fentlichen Leistungen von der Administration selbst beeinfluBt werden kann.

Dabei werden in der Literatur mehrere Kanäle der EinfluBnahme unterschieden, wobei das Ergebnis jedesmal eine überhöhte Nachfrage nach öffentlichen Leistungen und damit ein zu großes Budget ist. Die drei Arten der Einflußnahme, die nicht unabhängig voneinander sind, sind folgende:

1. Bürokraten haben auch ein Stimmrecht, sind Wähler und beeinflussen durch ihre Wahlentscheidung das AusmaB der staatlichen Leistungen.

2. Buirokratien haben eine wichtige Funktion bei der Formulierung von Programmen ("agenda setting").

3. Die Bürokratie hat ein spezifisches Informationsmonopl über die Auswirkungen der von ihr erbrachten Leistungen und kann dieses sowohl gegenüber den Politikern als auch den Wählern einsetzen.

\subsubsection{Zum Abstimmungsverhalten der Bürokraten}

Speziell TULLOCK,1) aber auch andere Autoren sehen einen Grund für einen wachsenden Staatsapparat darin, daß den Bürokraten (den Beschäftigten des öffentlichen Dienstes) die Möglichkeit gegeben ist, zu wählen. Diese haben aber ein Interesse an einem möglichst großen Budget, da sie gerade dadurch Umverteilungsgewinne machen können. Natürlich sind sie auch als Steuerzahler von einem zu hohen öffentlichen Haushalt negativ betroffen, aber ihr Gewinn aus der Bereitstellung öffentlicher Leistungen geht auf jeden Fall über den Wert hinaus, den sie diesen als reine "Konsumenten" zurechnen: Es handelt sich um eine Art

1) TULLOCK (1974) (1977) (1972) 
Selbstkontraktion, 1) da sie als Anbieter der öffentlichen Leistungen gleichzeitig durch die Wahlentscheidung über die Höhe des statiichen Sektors bestimmen können, somit als Nachfrager nach ihren eigenen Leistungen auftreten.

Die Effekte sollen an der nachfolgenden Abbildung dargestellt werden:2)

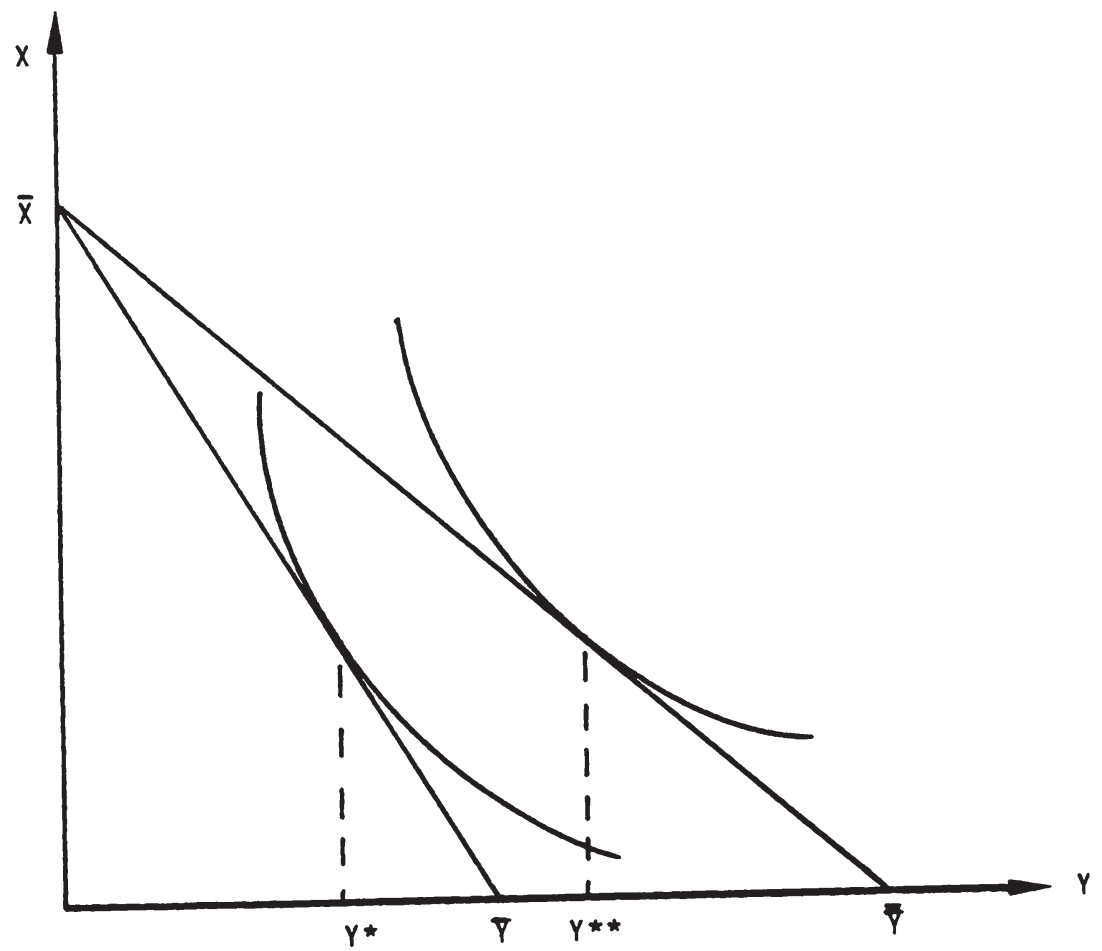

1) vgl. KRAUSE-JUNK (1972), S. 137

2) vgl. BORCHERDING/BUSH/SPANN (1977), S. 217 
In einer einfachen 2-Güterwelt soll die Entscheidung $z$ wischen einem öffentlichen Gut $Y$ und dem privaten Gut $X$ getroffen werden. Die Höhe des privaten Einkommens $X$ se $i$ vorgegeben, ebenso der marginale (= durchschnittliche) Steversatz.1) $Z$ u bestimmen ist nun, für welche Höhe des Angebots an öffentlichen Gütern das betrachtete Individuum stimmen wird. Hier soll dies die Menge $Y^{*}$ sein.

Das gilt allerdings nur, solange das betrachtete Individuum nicht selbst zur Bürokratie gehört. In diesem Fall soll die Höhe des privaten Einkommens abhängig sein von der Höhe des öffentlichen Outputs, beispielsweise durch höhere Bezahlung. Für das Individuum ist der Preis des öffentlichen Gutes kleiner als der marginale Steuersatz, beziehungsweise es zieht zwei Arten von Nutzen aus der Bereitstellung des öffentlichen Gutes: Einmal den direkten Nutzen aus dem Konsum des öffentlichen Gutes selbst, zum anderen die mit der Bereitstellung verbundenen Vorteile aus seiner Tätigkeit in der Administration. Ausgedrückt werden kann das durch eine Rechtsdrehung der Budgetgeraden auf $\bar{X} \overline{\bar{Y}}$. Die vom Bürokraten als Wähler nachgefragten Menge der öffentlichen Leistungen wird bei $Y \star \star$ liegen, das heiBt in aller Regel (Giffen-Paradox ausgeschlossen) bei einer höheren Menge, als wenn er ohne Beriucksichtigung des Umstandes, $d a B$ er vom öffentlichen BereitstellungsprozeB profitiert, seine Präferenzen offengelegt hätte.

Daraus folgt, daß die durch Wahlen geäuBerte Nachfrage nach öffentlichen Gütern bereits zugunsten eines zu hohen Budgets verzerrt ist, da bestimmte Wähler aus der Art der Bereitstellung selbst einen Nutzen ziehen.

i) Daraus bestimmt sich die Steigung der Budgetgerade $\bar{x} \bar{y}$ 


\subsubsection{Die Programmformulierung}

Durch die Formulierung von möglichen Programmen ergibt sich eine weitere Möglichkeit für die Bürokratie, das Ergebnis des politischen Prozesses zu bestimmen. Ausgangspunkt dieser Modelle des "agenda-setting"l) ist die oberlegung, daB die Budgetvorschläge, die zur Wahl gestellt werden, von der Bürokratie vorbereitet werden. Dabei kann nur eine begrenzte Anzahl von Alternativen zur Wahl gestellt werden. Die Bürokratie hat somit die Chance, durch die Ausgestaltung der verschiedenen Vorschläge dafür $z u$ sorgen, daß der ihr genehme bei einer Wahlentscheidung gewinnt.

Das Grundkonzept soll an einem extrem vereinfachten eindimensionalen Median-Wähler-Modell dargestellt werden.2) Dabei soll die Entscheidung über eine bestimmte Höhe des Budgets getroffen werden, das stellvertretend für ein Bündel öffentlicher Leistungen stehen soll. Auch die Steuerlastverteilung soll vorgegeben sein. Des weiteren soll angenommen werden, $d a B$ die wähler eine gleiche positive wie negative Abweichung von dem von ihnen als optimal angesehenen Budget gleich stark bewerten, das heiBt: Eine $10 \%$ ige Ober-oder Unterschreitung wird von ihnen gleich schlecht angesehen. Als Gleichgewichtslösung ergibt sich dann nach der herkömmlichen Lösung das Budget, das der Median-Wähler bevorzugt.

i) DENZAU, MACKAY, WEAVER (1979) (1981); DENZAU, MACKAY (1980); MACKAY, WEAVER (1978) (1979); ROMER, ROSENTHAL (1978) (1979); FILIMON, ROMER, ROSENTHAL (1982)

2) DazU: DENZAU, MACKAY, WEAVER (1979) 


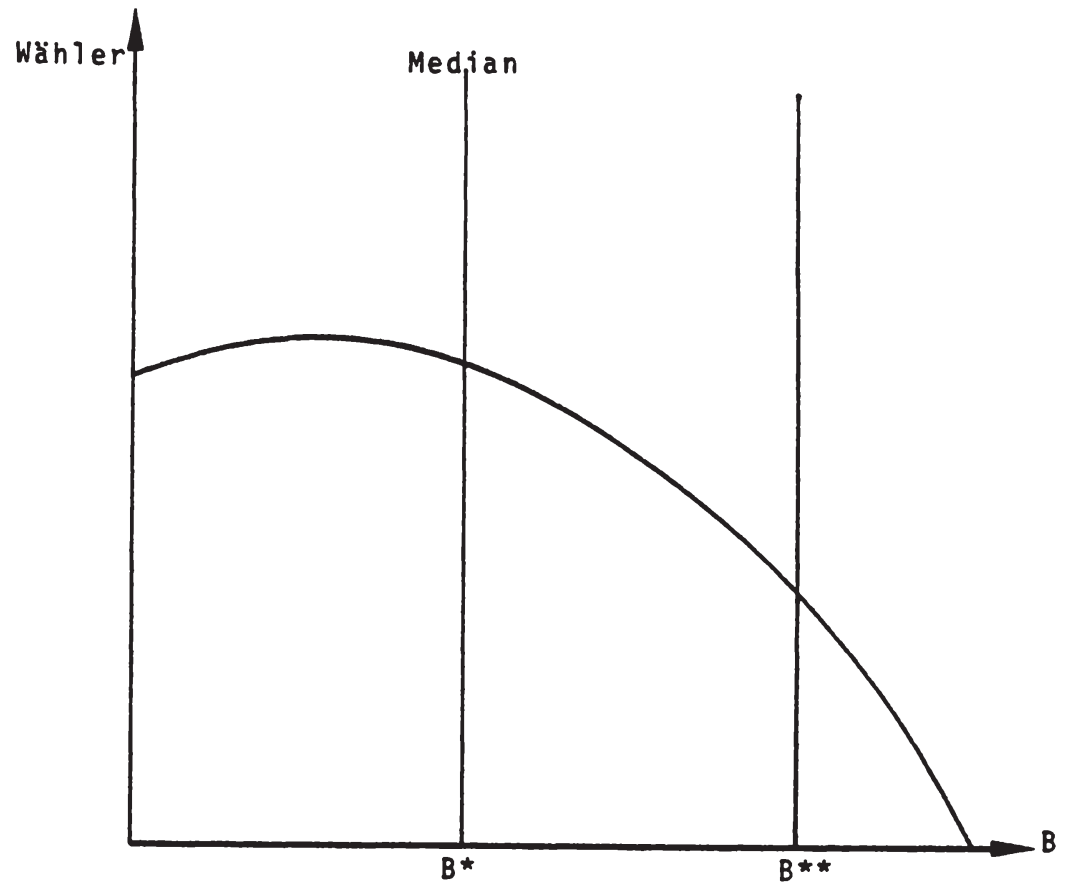

Auf der Abszisse sei die Höhe des Budgets abgetragen, auf der Ordinate der Anteil der Wähler (in Prozent ausgedrückt), die das jeweils vorgeschlagene Budget für das Beste halten. Als Gleichgewichtslösung ergibt sich dann 
das Budget $B^{*}$, die BudgetgröBe, die der Median-Wähler bevorzugt. Dieses Budget wird gegen jede Alternative gewinnen.

Dies gilt allerdings nur, wenn im Rahmen eines politischen Wettbewerbs eine Annäherung der tatsächlichen Vorschläge an das Median-Wähler-Budget möglich ist. Ausgangspunkt der Analyse der genannten Autoren ist nun, daB die Teilnahme am politischen ProzeB nicht kostenlos und somit nicht uneingeschränkt möglich ist. Teilnahme am politischen ProzeB beinhaltet aber in diesem Zusammenhang die Möglichkeit, Budgetvorschläge zu machen und abstimmen zu lassen.

Damit besteht ein bestimmter monopolistischer Spielraum bei der Aufstellung des Budgets beziehungsweise der verschiedenen Budgetvorschläge, und damit die Möglichkeit, das Wahlergebnis durch die konkrete Ausgestaltung der zur Abstimmung gestellten Alternativen zu beeinflussen. Geht man davon aus, daß die statiche Bürokratie als die Instanz, die im wesentlichen mit der vorbereitung und Formulierung von Programmen und Budgetvorschlägen betraut ist, diese monopolistische stellung innehat, und daß diese an einem möglichst hohen Budget interessiert ist, dann ergibt sich folgendes Problem: Im einfachsten Fall kann die Burokratie einen Budgetvorschlag zur Wahl stellen und als Alternative nur ein Budget in Höhe von Null zulassen, das heiBt die Wähler vor eine "Alles-oder-nichts-Entscheidung" stellen. Die maximal durchsetzbare Budgethöhe liegt dann bei $B^{\star \star}$, dem Budget, das der Medianwähler gerade noch einem Budget in Höhe von Null vorzieht. Genau dieses Budget wird von der Birokratie als Vorschlag unterbreitet. Der Budgetumfang wird auf jeden Fall weit gröBer sein als der, der sich in einem "normalen", sprich konkurrenzmäBigen, politischen Entscheidungsprozeß herausgebildet hätte. 
Das vorgestellte Modell ist durch seine Annahmen über die Eindimensionalität extrem einfach, aber auch die Aufgabe dieser vereinfachenden Annahmen ändert nichts an der Grundargumentation, $d a B$ ein monopolistischer Spielraum der Bürokratie bei der Aufstellung des Budgets zu einem aus der Sicht der Wähler zu hohen und damit ineffizienten Staatshaushalt fuihrt.

Eine mögliche Modifikation besteht darin, daß die Bürokratie nicht eine Alles-oder-nichts-Entscheidung herbeiführen kann, sondern daB sie nur einen Kostenvorteil bei der Formulierung des Haushaltsvorschlags hat. Unter diesen Umständen muß sie damit rechnen, daB, wenn der eingebrachte Budgetvorschlag zu hohe (Effizienz-) Kosten für die Wähler mit sich bringt, diese die Kosten eines Gegenvorschlags auf sich nehmen, einen solchen formulieren und zur Abstimmung stellen. Diese Möglichkeit begrenzt $z$ war den Spielraum der Bürokratie und führt dazu, daB das Budget nicht zu extremen GröBen anwächst; solange jedoch ein Kostenvorteil bei der Formulierung des Budgetvorschlags auf Seiten der Burrokratie vorhanden ist, wird eine Tendenz zum ausschlieBlichen Angebot von zu groBen Budgets zu verzeichnen sein.1)

Eine weitere Modifikation ergibt sich, indem man die Idee des Budgets als einer homogenen Größe aufgibt und eine unterschiedliche Zusammensetzung der öffentlichen Leistungen und deren Finanzierung zuläBt. Damit ergibt sich für die Bürokratie ein weiteres Instrument, um eigene Vorstellungen über die Höhe der Statsleistungen durchzusetzen: Der gezielte diskriminierende Einsatz von Statsleistungen und Steuern. ${ }^{2}$ )

i) vgl. DENZAU, MACKAY, WEAVER (1981)

2) "benefit and tax share discrimination"; $v g l$ besonders DENZAU, MACKAY (1980), MACKAY, WEAVER (1978), HUNTER (1983) 
Der Grundgedanke ist dabei der folgende: Die Höhe des Steuersatzes kann als Preis, den der einzelne Stimmbürger für die öffentlichen Leistungen zahlen muß, angesehen werden. Kennt die Bürokratie die verschiedenen Zahlungsbereitschaften der Wähler, kann sie durch Steuersatzdiskriminierung und gezielte Differenzierung der statilichen Leistungen die Zustimmung der Bürger zu einem möglichst hohen Budget erreichen. Diejenigen Steuerzahler, deren Nachfrage nach öffentlichen Leistungen stark (Steuer-) preiselastisch ist, werden mit einem relativ geringen Steuersatz belastet bzw. erhalten einen recht hohen Anteil an den Statsleistungen. Die Stimmbiirger mit einer preisunelastischen Nachfrage nach öffentlichen Leistungen werden hingegen sehr stark mit Steuern belastet, da sie trotzdem für die Erstellung der öffentlichen Leistung stimmen werden. Durch geschickte Diskriminierung läßt sich so ein maximales Budget durchsetzen.

Eine Steuersatzdiskriminierung selbst ist dabei nicht unbedingt negativ zu bewerten: auch die Lindahl-Lösung einer optimalen Versorgung mit öffentlichen Giitern verlangt ja nach marginaler Zahlungsbereitschaft differenzierende Steuersätze. Die Steuerlastverteilung, die die Bürokratie wählt, unterscheidet sich jedoch von der nach der Lindahl-Lösung. Die Bürokratie wird nicht nach marginalen, sondern nach totalen (Gesamt-) Zahlungsbereitschaften differenzieren, um ein möglichst hohes Budget durchzusetzen: Individuen, die dem bestehenden Umfang an staatlichen Leistungen einen relativ hohen Wert beimessen, werden mit hohen Steuern belastet, auch wenn sie einer weiteren Einheit öffentlicher Güter nur einen geringen Wert beimessen, und umgekehrt. Im Ergebnis wird das Budget unter allokativen Gesichtspunkten zu groB sein (auBer im 
Fall vollkommen preisunelastischer Nachfrage), und die Struktur der Steuerbelastung in aller Regel auch von der Lindah1-Lösung abweichen.1)

Analog kann für eine differenzierte Zuteilung öffentlicher Leistungen (soweit diese nicht rein öffentliche Güter darstellen, also von allen im gleichen $A u s m a B$ konsumiert werden) argumentiert werden: Hier wird die Bürokratie den Bürgern, deren Nachfrage nach öffentlichen Leistungen relativ preiselastisch ist, eine größere Menge zuteilen als den Bürgern mit relativ preisunelastischer Nachfrage, da bei letzteren eine Ausdehnung des Angebots an Staatsleistungen nur zu einer geringfügig erhöhten (Steuer-) zah-

1) Diese Argumentation weist Parallelen auf zu der Polemik BUCHANAN/BRENNAN's (1980) gegen die Möglicheit von Steuersatzdiskriminierungen im Leviathan-Staat. In deren Modell steht jedoch der steueraufkommenmaximierende Stat im Mittelpunkt der Analyse, der ohne irgendwelche exogenen Beschränkungen durch differenzierende Besteuerung verschiedener Individuen ein möglichst hohes Aufkommen zu erreichen sucht. Die Steuersatzdiskriminierung dient bei ihnen der maximalen Ausbeutung der staatsbürger, wird also nach den unterschiedlichen Substitutionsmöglichkeiten der Individuen vorgenommen, während bei DENZAU, MACKAY und WEAVER die Diskriminierung der Maximierung der politischen Zustimmung zu den jeweiligen Budgetvorschlägen dient. Die unterschiedlichen Reaktions-bzw. Ausweichmöglichkeiten der Steuerzahler spielen dabei keine Rolle. 
lungsbereitschaft führt. Auch durch solche MaBnahmen versucht die Bürokratie, die Zustimmung zu einem relativ großen Budget zu erreichen.1)

Grundlegend für alle diese oberlegungen ist, $d a \beta$ die staatliche Bürokratie die Initiative bei der Formulierung der Ausgaben - und Einnahmenprogramme hat. Begründet werden kann dies mit der Unzulänglichkeit der Formulierung der Budgetaufstellung durch nicht-statiche Stellen. Tatsächlich dürfte es für AuBenstehende unmöglich bzw. zu teuer sein, mit der gleichen Kompetenz und Sachkenntnis einen Alternativhaushalt zu dem von der staatlichen Bürokratie vorgeschlagenen zu entwickeln. Auch wenn verschiedene Parteien um den Wahlsieg konkurrieren, bedient sich die jeweils siegreiche im wesentlichen eines gleichbleibenden Verwaltungsapparats und ist somit ebenso von den Initiativen der Bürokratie abhängig. Die Alternativvorschläge, die die einzelnen Parteien den Wählern vorlegen, beziehen sich entsprechend nie auf die Gesamtheit aller Detailprobleme - eine echte Alternative in diesem Sinne wäre nur zu erwarten, wenn jede Partei einen eigenen vollständigen Staatsapparat besäße, der im Fall des Wahlsiegs dann die Aufgaben der staatlichen Bürokratie übernehmen würde.

i) Die Möglichkeit einer Leistungsdiskriminierung setzt allerdings voraus, $d a B$ die betreffenden öffentlichen Leistungen nicht wieder auf privaten Märkten weiterverkauft werden können. In diesem $F$ all wird sich ein einheitlicher Preis für das entsprechende Gut bilden, eine differenzierende Leistungsabgabe durch die Bürokratie ist sinnlos. Wenn jeder das öffentliche Gut auf dem Markt erwerben kann, kann die Bürokratie durch eine nach Zahlungsbereitschaft differenzierte Leistungsabgabe kein höheres Budget durchsetzen. Vgl. dazu die Diskussion zwischen DENZAU/MACKAY (1980) und STAAF/WEST (1980) 
Der Spielraum der Bürokratie bei der Programmformulierung ist dementsprechend nicht unbedingt unbegrenzt, ein Spielraum ist aber auf jeden Fall vorhanden.

\subsubsection{Bürokratie und Information}

Eine wichtige Rolle für die Herleitung der Machtposition der Bürokratie dürfte auch die Verfügbarkeit von Informationen spielen. Die Bedeutung mangelnder Information uiber die Wählerwünsche und die Auswirkungen spezieller Programme auf Seiten der Politiker wurde bereits angesprochen.1) Hier geht es allerdings weniger um die Information über die Effizienz der bürokratischen Produktion, sondern um die Möglichkeiten, die die Administration hat, um durch gezielte Informationspolitik die Nachfrage nach statlichen Leistungen zu erhöhen.

GALBRAITH (1958) hat bei seiner Begriundung der Unterversorgung der Gesellschaft mit öffentlichen Gütern auf die Möglichkeiten der Informationspolitik hingewiesen. Seine These lautet, daß durch die Werbung für privat produzierte und angebotene Güter die Nachfrage der Individuen systematisch zuungunsten der öffentlichen Leistungen verzerrt würde, es somit zu einem zu kleinen öffentlichen Sektor käme. GALBRAITH übersieht dabei, daB auch die Administration als Anbieter staatlicher Leistungen Werbung treiben kann. 2)

i) $\mathrm{vg} 1.3 .3 .2$.

2) vgl. auch TUERCK (1978) S. $72 f f$. 
AnnahmegemäB ist die Bürokratie an einem möglichst hohen Budget interessiert. Entsprechend liegt es in ihrem Interesse, die Lasten der öffentlichen Einnahmeerzielung möglichst gering und den Nutzen der öffentlichen Ausgaben möglichst hoch erscheinen zu lassen.1)

Die Möglichkeit, daB gerade durch Werbung die Nachfrage nach öffentlichen Leistungen erhöht werden kann, ergibt sich nach WAGNER (1978) aus den Eigenschaften dieser spezifischen Leistungen, welche sich im AnschluB an DARBY/KARNI (1973) als "credence services", "Vertrauensgitter" bezeichnen lassen. Das sind solche Leistungen, die zwar eine bestimmte Auswirkung auf die Nutzenposition der Nachfrager haben, bei denen es aber nicht oder nur sehr eingeschränkt möglich ist, diese auch genauer zu spezifizieren. Solche gibt es auch als privates Angebot. Als Beispiel mag ein Arzneimittel dienen: In den seltensten Fällen läßt sich für den einzelnen der sich nach Einnahme des Arzneimittels einstellende Gesundheitszustand mit Sicherheit auf die Wirkungen dieses bestimmten Medikaments zurückführen. Eine Gesundheitsverbesserung wäre möglicherweise auch ohne dieses Medikament zustandegekommen, bzw. der Krankheitsverlauf hätte ohne die Einnahme dieser Arznei noch schlimmer verlaufen können, so daB auch eine Verschlechterung des Gesundheitszustandes nicht unbedingt einen SchluB auf die Wirksamkeit des Mittels zuläBt. WAGNER argumentiert nun, daB die öffentlichen Leistungen nicht unbedingt von ihrem spezifischen Charakter her "credence services" darstellen, sondern durch die Art der Bereitstellung dazu werden

Dieses Argument läBt sich in die Begriffe des in Abschnitt 3.3.2 dargestellten Informationskalküls übersetzen: LäBt sich bei einem privat angebotenen Gut die Auswirkung auf

i) vgl. dazu WYSE/DAVIES (1978), WAGNER (1978) 
die eigene Nutzenposition noch relativ einfach feststellen, indem auf den Konsum dieses Gutes verzichtet und somit eine Vergleichsmöglichkeit geschaffen wird, diurfte bei öffentlichen Leistungen diese option nicht mehr vorhanden sein: Ein zeitweiliger Verzicht auf Steuerzahlungen wird auf erhebliche Schwierigkeiten beim Finanzamt stoßen, ebenso wie ein individueller Verzicht auf den durch Polizei und Justiz garantierten Schutz vor Verbrechen kaum vorstellbar ist.

Selbst bei öffentlichen Leistungen, bei denen ein SelbstausschluB möglich ist, ist diese Art des Tests eine recht kostspielige Angelegenheit, da der Verzicht auf die Inanspruchnahme öffentlicher Leistungen in der Regel nicht mit einer verringerten Steuerbelastung verknüft ist, d.h. anders als bei einer Transaktion auf Privatmärkten der Kaufpreis nicht einbehalten werden kann. Zudem hat der individuelle Verzicht auf die Inanspruchnahme öffentlicher Leistungen andere Auswirkungen als die Einstellung der Leistungen insgesamt, ein $S c h l u B$ läBt sich nicht ohne weiteres ziehen.

Die Essenz ist, daß die Informationskosten bei öffentlichen Leistungen allein durch die spezifische Art der Bereitstellung schon höher liegen als bei privaten Gütern. Dadurch werden solche Leistungen tendenziell zu "credence services".

Die spezifischen Eigenarten der "credence services" bieten dem Anbieter die Möglichkeit, durch falsche Informationen uber die Qualität oder den notwendigen Umfang der Leistungen die Nachfrage zu beeinflussen bzw. in die Höhe zu treiben. Die Bürokratie hat somit die Möglichkeit, durch eine spezifische Informationspolitik, also Werbung, einen positiven Nutzen der öffentlichen Leistungen zu suggerieren und somit für eine künstliche Nachfrageerhöhung zu 
sorgen.1) Werbung zu treiben nimmt dabei natülich ebenso Ressourcen in Anspruch wie andere Tätigkeiten. Die Administration wird also solange Werbung für die öffentlichen Leistungen treiben, bis die Grenzkosten der Werbung2) gleich den Grenzerträgen aus der Erhöhung der Nachfrage nach öffentlichen Leistungen, also des erhöhten Budgets, sind.

3.3.6 Die Auswirkung von Budgetbeschränkungen auf den politischen ProzeB

Eine Möglichkeit, die Auswirkungen dieser Art des Statsversagens auf der Nachfrageseite zu vermindern, könnte in der Einführung von Budgetbeschränkungen gesehen werden. Der Sinn einer solchen Maßnahme ist dann immer einsichtig, wenn das bestehende Budget, gemessen an den Wuinschen der Wähler, ubberdimensioniert ist.

Allerdings stellt nicht die Höhe des Budgets an sich die ZielgröBe dar, die durch die Einführung einer Budgetgrenze beeinfluBt werden soll. Es geht vielmehr um eine Reduktion der Ineffizienz des staatlichen Handelns. Eine Budgetbegrenzung hat dann einen Sinn, wenn das individuelle Entscheidungskalkiil der am politischen ProzeB Beteiligten

i) Das soll nicht heiBen, daß jede Werbung für öffentliche Leistungen auf einen zu hohen Umfang des Budgets gerichtet ist. Ohne Werbungsmaßnahmen kann die Nachfrage wegen der Nichtkenntnis der Effekte der öffentlichen Leistungen auch zu gering ausfallen.

2) Beispielsweise durch eine Verringerung des diskretionären Budgets 
so beeinfluBt wird, $d a B$ es zu einer Verminderung der Ineffizienzen kommt.1) ob dies gegeben ist, soll im folgenden geprüft werden.

Bei einer nicht-existierenden Budgetgrenze werden die Interessengruppen solange politische Transfers auf Kosten der relativ uninformierten Mehrheit durchsetzen, bis der marginale Wert der letzten in die transfererwerbende Aktivität eingesetzten Ressource gleich ist dem Wert der dadurch erreichten Transfers (oder der vermiedenen Belastung). Dabei hängt die Höhe der notwendigen Ressourcen ab von dem Einsatz von Ressourcen a) der ebenfalls um Transfers sich bemühenden anderen Interessengruppen und $b$ ) den Ressourcen, die die einzelnen Bürger zur Informationsgewinnung in den politischen ProzeB investieren.

Die einzelnen Interessengruppen stehen dabei in einem kompetitiven Verhältnis zueinander. Je größer das Budget bereits ist, desto schwerer ist eine weitere marginale Erhöhung zwecks Gewinn von Transfers durchzusetzen, d.h., desto höher sind die Kosten der Transfererlangung. Die Aktionen anderer Interessengruppen tangieren ceteris paribus die eigenen Möglichkeiten negativ.

Dennoch ist die dadurch zustandekommende Beschränkung nicht so stark, da eigene Transfers durch Budgeterhöhungen durchgesetzt werden können, die nicht zu einer direkten Verminderung der für andere Interessengruppen zur Verfügung stehenden Beträge führt.

Dies ändert sich, wenn eine quantitativ bindende Budgetbeschränkung eingeführt wird. Jede Erhöhung des für eigene Zwecke verwandten Budgets führt dann zwangsläufig zu einer Verminderung der für die konkurrierenden Interessengruppen

i) In diesem Sinn MUSGRAVE (1981) und ARANSON/ORDESHOOK (1981) 
zur Verfuigung stehenden Beträge. Damit treten die einzelnen Interessengruppen in eine direkte Konkurrenz zueinander. Nicht mehr die marginale Widerstandsbereitschaft der Wähler ist die relevante Größe, sondern die um dasselbe Budget konkurrierenden Inputs anderer Interessengruppen. Das hat Auswirkungen sowohl auf die Höhe der Ressourcen, die zur Erlangung von Transfers eingesetzt werden, als auch auf die Ausgestaltung der Statsausgaben, über die die Interessengruppen ihre Gewinne erzielen.

\subsubsection{Die Ressourcen zur Transfererlangung}

Eine Budgetbeschränkung fuihrt zu einer Erhöhung der Intensität des Wettbewerbs um Transfers. Das bedeutet, daB für alle am politischen ProzeB Beteiligten die Kosten der Transfererlangung steigen. Der Preis des politischen Einkommens steigt, und es steht zu vermuten, daß das zu einer geringeren Nachfrage nach politischem EinfluB führen duirfte. Eine Einführung von Budgetgrenzen könnte unter diesen Umständen zu einer Reduzierung der Ausgaben führen, die für politische Maßnahmen benutzt werden. Die erwarteten Erträge einer Investition in den politischen Entscheidungsprozeß sinken.

Damit würde eine Budgetbegrenzung zu einem positiven Effekt führen: Die für die spezifische Entscheidung verwandten Ressourcen sinken und können anderen, produktiven Verwendungen zufiieBen - eine Umschichtung von Ressourcen von "rent-seeking" zu "profit-seeking".

Die Einführung einer Budgetbeschränkung kann dann interpretiert werden als eine Verschiebung der Ertragsfunktion des politischen Einflusses: die politischen Umverteilungsgewinne bei gegebener EinfluBnahme sinken. Die Effekte sollen an folgender, in Abschnitt 3.3.4.1. 
beschriebener Zeichnung verdeutlicht werden:

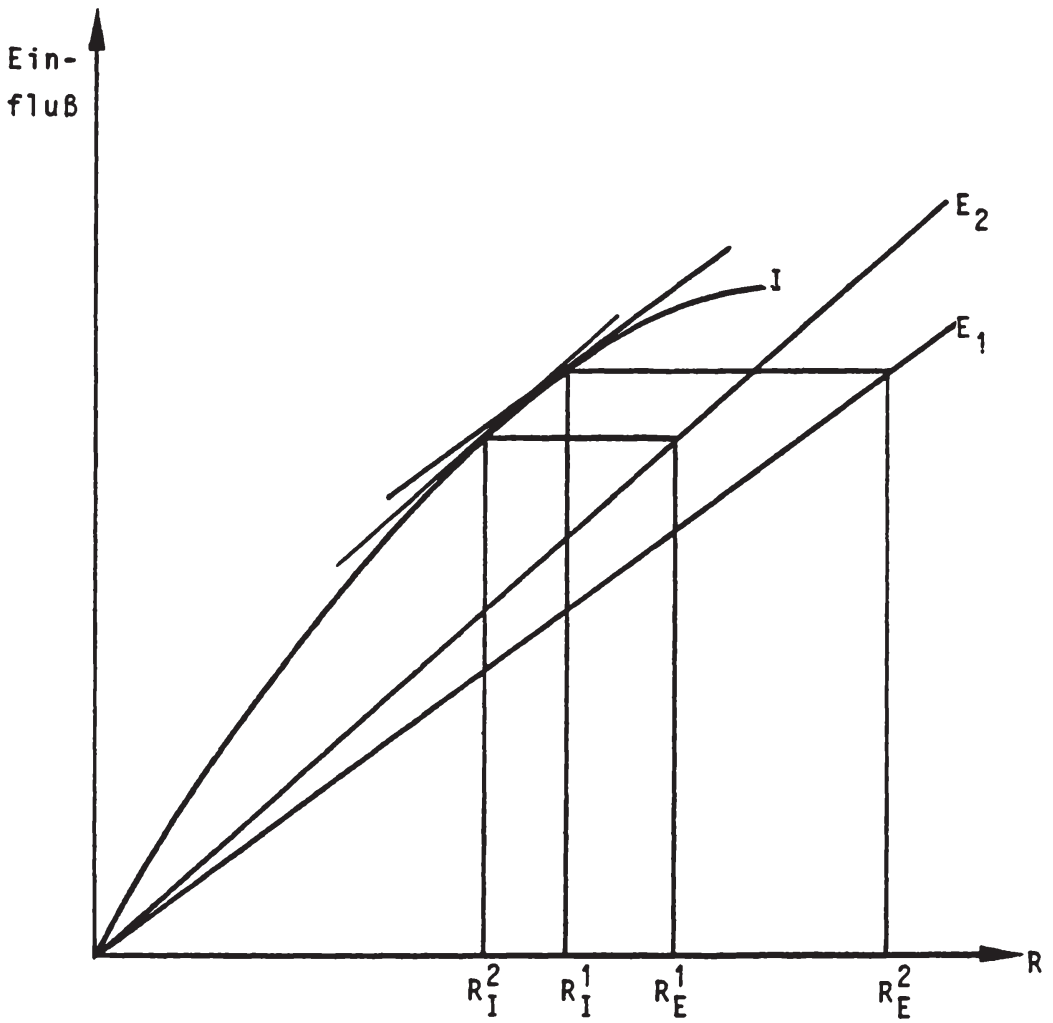

I bezeichnet dabei die Höhe des Einflusses in Abhängigkeit vom Ressourceneinsatz, E 1 die Erträge ohne und $E_{2}$ die Erträge nach Einführung der Budgetbegrenzung. Ein Gleichgewicht ergibt sich da, wo Grenzkosten und Grenzerträge der Einflußnahme gleich sind. Wurden ohne Budgetbegrenzun- 
gen noch Ressourcen in der Höhe von $R_{I}{ }^{1}$ in den politischen ProzeB investiert, so sinken diese nach Einführung der Budgetgrenze auf $R_{I}^{2}$.

Die postulierten Folgen auf die Kosten der Einflußnahme auf den politischen ProzeB sollen noch einmal im ZweiGruppen-Beispiel verdeutlicht werden.

Durch eine Budgetbeschränkung ergibt sich eine maximale Höhe der im politischen ProzeB zu gewinnenden oder zu verlierenden Ressourcen. $T r_{i}$ ist also nach oben und unten auf einen Maximalbetrag $T r_{m a x}$ begrenzt. Damit verändert sich in gewissen Grenzen die Anpassungsfunktion der Interessengruppen $i$ und $j$. Folgende Zeichnungen sollen das verdeutlichen.

\section{Gruppe i}

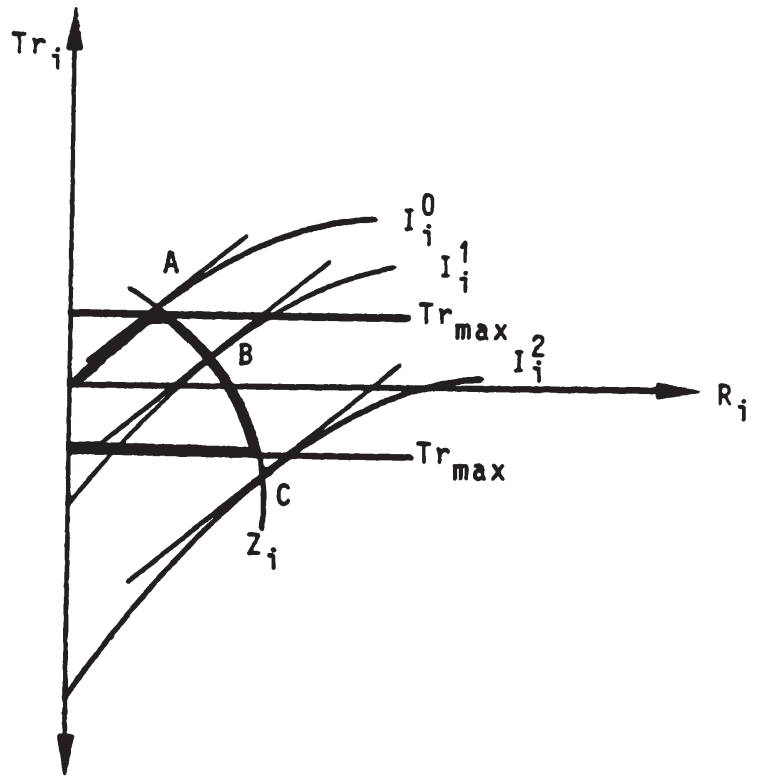




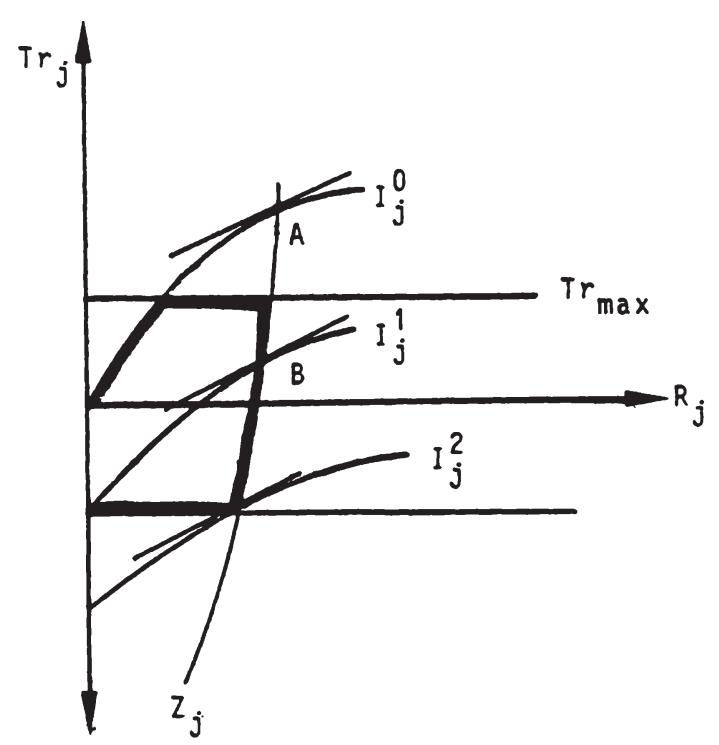

Die Budgetbeschränkung wirkt sich natürlich nur dann aus, wenn sie bindend wird. Betrachtet man die Kurve $I^{0} i$, die die Höhe der Transferzahlungen in Abhängigkeit vom Ressourceneinsatz der Gruppe $i$ bei gegebenem, sehr geringen Ressourceneinsatz durch Gruppe $j$ beschreibt, so ergibt sich folgendes: Den maximalen Nettogewinn macht Gruppe $i$ in Punkt $A$. Dazu muB sie eine bestimmte Menge Ressourcen in den politischen ProzeB einsetzen und ein Transfervolumen realisieren, das über dem im Rahmen der Budgetbeschränkung möglichen liegt. Da die Budgetbeschränkung nicht durchbrochen werden darf, muB Gruppe $i$ sich mit einem geringeren Transfervolumen begnügen, braucht aber andererseits auch nur eine geringere Menge an Ressourcen in den Prozeß der politischen EinfluBnahme zu investieren. Die Budgetbeschränkung führt dazu, daB der Einsatz von Ressourcen in den politischen ProzeB sinkt. 
Bei einem höheren Ressourceneinsatz durch $j$ soll als neue Ertragsfunktion der EinfluBnahme die Kurve $I^{1} ;$ gelten. Hier liegt das gleichgewichtige Transfervolumen unterhalb der Budgetgrenze (Punkt $B$ ). In diesem Fall bleibt die Höhe des Ressourceneinsatzes unverändert.

Bei einem sehr hohen Ressourceneinsatz durch $j$ (Kurve $I^{2} i$ ) wäre es in der Ausgangslage sogar dazu gekommen, daß Gruppe $i$ Umverteilungsverluste erlitten hätte, deren Höhe über dem maximalen Budget liegt (Punkt $C$ ). Die von Gruppe $i$ eingesetzten Ressourcen dienten dann nur dazu, den Umverteilungsverlust nicht noch gröBer werden zu lassen. Dies erübrigt sich jetzt dadurch, daB der maximale Verlust durch die Budgetbeschränkung vorgegeben ist. Wenn durch Einflußnahme die Verluste nicht verringert werden können, dann kann getrost darauf verzichtet werden, Ressourcen in den politischen ProzeB einzusetzen. Für Gruppe j gelten analoge oberlegungen.

Die modifizierten Anpassungskurven lassen sich somit folgendermaßen darstellen:

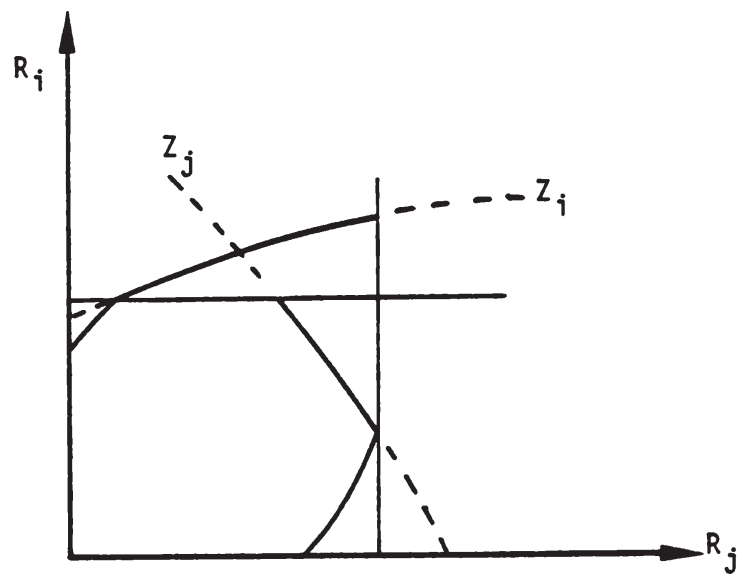


Vorausgesetzt, die Budgetbegrenzung ist gegenüber dem Ausgangsbudget bindend (sonst ist sie sinnlos), dann folgt daraus, daB eine Gruppe auf die EinfluBnahme verzichtet - sie kann sich dadurch nicht verschlechtern. Damit wird der anderen Gruppe ermöglicht, mit einem Minimum an eigenen Ressourcen das Budget zu ihren Gunsten auszuschöpfen. Der Einsatz von Ressourcen in den ProzeB der EinfluBnahme auf politische Entscheidungen wird damit reduziert, eine Budgetbeschränkung hätte in diesem $F$ all einen positiven Effekt. Allerdings können diese Ergebnisse nicht auf den Mehr-Gruppen-Fall übertragen werden. Im Zwei-Gruppen-Modell ergibt sich ja per definitionem, falls die Budgetbeschränkung bindend wird, daß eine Gruppe der einzige Gewinner des Prozesses ist und bereits in der Ausgangssituation einen Umverteilungsgewinn in Höhe des Gesamtbudgets, also einen höheren Betrag als unter der Budgetbegrenzung möglich, realisierte. Da die Einflußnahme der anderen Gruppe mehr oder weniger darauf gerichtet war, diese Umverteilungsgewinne nicht noch weiter wachsen zu lassen, und dies durch die Einführung einer Budgetbegrenzung bereits verhindert wird, ist ein sinken der in den politischen ProzeB eingesetzten Ressourcen nur logische Konsequenz.

Dieser einfache Zusammenhang besteht nicht mehr, wenn wir eine Vielzahl von Interessengruppen betrachten. Immer noch begrenzt die Budgetbeschränkung die Höhe der Umverteilungsgewinne nach oben - dies gilt aber für die Gesamthöhe der politischen Einkommen, nicht für jeden einzelnen Transferbetrag.

$D a B$ die Gewinne insgesamt geringer werden und damit die Erträge der für die politische EinfluBnahme eingesetzten Ressourcen sinken, impliziert aber noch nicht, daB deswegen weniger Ressourcen eingesetzt werden. Im Gegenteil kann die Verschärfung der Konkurrenzsituation, die 
durch die Budgetbeschränkung zustande kommt, dazu führen, daß die Höhe der Einflußnahmekosten noch steigt. AnnahmegemäB ist die Höhe des Einflusses einer Interessengruppe bei gegebenen in den politischen ProzeB eingesetzten Ressourcen nicht unabhängig von der Menge der Ressourcen, die andere Interessengruppen aufwenden. Mit anderen Worten: die Lage der "Produktionsfunktionen politischer Einflußnahme" sind nicht unabhängig voneinander, es kommt zu reziproken externen Effekten. Diese dirften in aller Regel nicht separabel sein, d.h. auch die Grenzerträge der in den politischen ProzeB eingesetzten Ressourcen dirfte von der Höhe der EinfluBnahme anderer Gruppen abhängen.1)

Damit kann aber die Produktionsfunktion politischer Einflußnahme fur eine bestimmte Interessengruppe immer nur bei gegebenen in den politischen ProzeB eingesetzten Ressourcen der anderen Gruppen abgeleitet werden. Die Einführung einer Budgetbegrenzung verändert nun das Gefüge der Höhe der gegenseitigen Abhängigkeiten des Ressourceneinsatzes in den politischen ProzeB. Anders ausgedruckt: Die jeweilige Produktionsfunktion verändert nicht nur ihre Lage, sondern auch ihre Form. Dann ist aber nicht mehr eindeutig prognostizierbar, ob eine Budgetbeschränkung zu einer Erhöhung oder Verminderung des Einsatzes von Ressourcen in den politischen ProzeB führt. Dies soll in der folgenden Zeichnung demonstriert werden.

i) vgl. BECKER (1983) S. $388 \mathrm{ff}$. Möglicherweise gibt es dann kein Gleichgewicht, davon soll aber hier abstrahiert werden. Vgl. dazU DAVIS/WHINSTON (1962) 


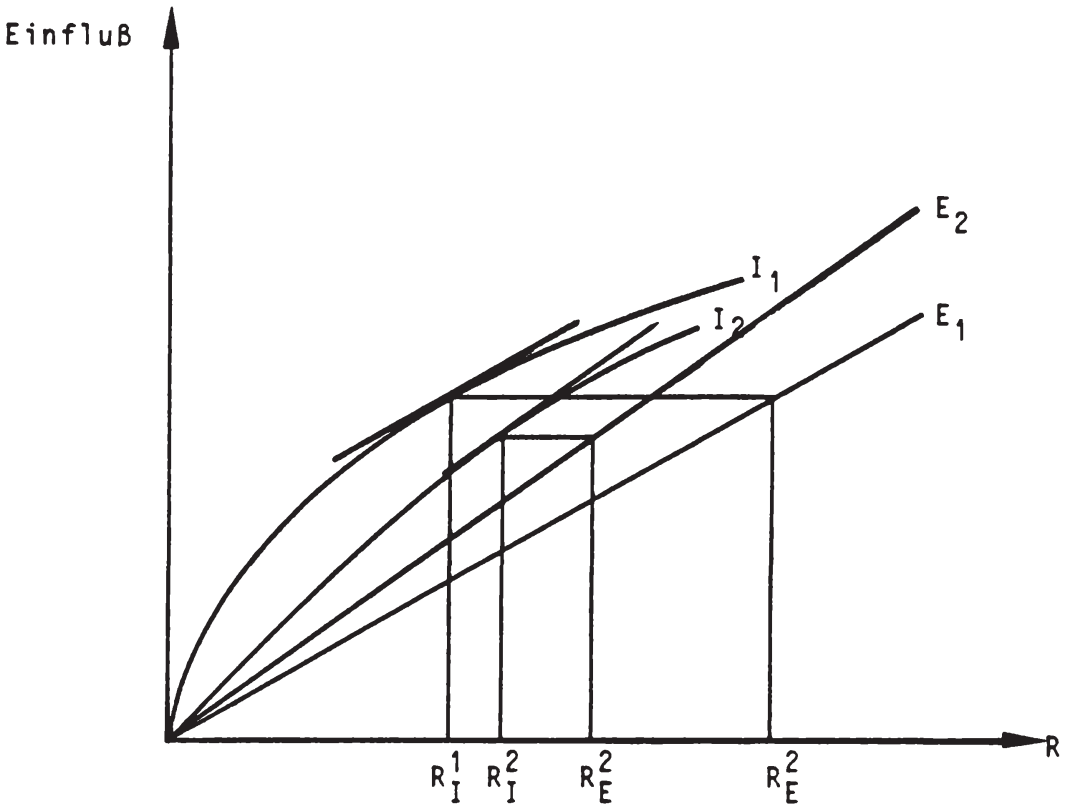

I 1 symbolisiert die Produktion politischen Einflusses für eine Interessengruppe in einer Situation, in der keine Budgetbeschränkung gilt, I 2 die Produktionsfunktion mit einer Budgetbeschränkung, jeweils bei gegebener EinfluBnahme anderer Gruppen. Insgesamt ergibt sich jedoch unter dem Regime einer Budgetbeschränkung ein erhöhter Ressour ceneinsatz durch die Interessengruppe ( $R_{I}{ }^{2}$ statt $R_{I}{ }^{1}$ ), obwohl die Umverteilungsgewinne $\left(R_{E}{ }^{2}-R_{I}^{2}\right.$ gegenüber $R_{E}{ }^{1}$ $R_{I}{ }^{1}$ ) bzw. die Höhe der Ausgaben, die der Interessengruppe zugute kommen $\left(R_{E}^{2} b z w . R_{E}^{1}\right)$, gesunken sind. 
Naturlich ist es keineswegs $z$ wingend, daB die reziproke Abhängigkeit des Einflusses der Interessengruppen zu diesem Ergebnis fuihrt - das Gegenteil ist aber auch nicht zwingend. Als a-priori-Aussage läBt sich somit nur festhalten, daB die Wirkung einer Einführung von Budgetgrenzen auf den Einsatz von Ressourcen zur Entscheidungsfindung nicht eindeutig vorhersagbar ist: eine Budgetbegrenzung kann die prozeBbezogene Ineffizienz reduzieren, kann sie aber auch erhöhen.

3.3.6.2 Reduktion oder Erhöhung situationsbezogener Ineffizienz?

Eine zweite Auswirkung der Budgetbeschränkung ergibt sich aus der Art und Weise, wie budgetäre Transfers zu erlangen sind. Hier spielt folgender Gesichtspunkt eine Rolle: Im ProzeB ohne Budgetbeschränkung stellt sich für die einzelnen Interessengruppen das Entscheidungsproblem, inwieweit eine Erhöhung der eigenen Transfers durch eine Erhöhung des "Ineffizienzgrades" des Budgetprozesses erreicht werden kann.1) Im Fall einer Budgetbeschränkung ändert sich dieses Kalkül, gegebenenfalls sogar das Vorzeichen. Jede Erhöhung des für eigene Zwecke zur Verfügung stehenden Budgets führt jetzt zu einer Reduktion der budgetären Spielräume anderer Interessengruppen. Hier steigen offensichtlich die Opportunitätskosten, und eine Erhöhung der budgetären Ineffizienz wird zu einer Strategie minderer Qualität. Durch eine Budgetgrenze ist zumindest $z u$ erwarten, daß eine Erhöhung der Ineffizienz, um Verteilungsgewinne zu erzielen, eine weniger erfolgversprechende Strategie darstellt, und somit in geringerem Maße angewandt wird.

i) Vgl. dazu Abschnitt 3.3 .4 .3 
Die Problematik, daB eine Ausdehnung der Ausgaben ibber die Höhe der eigentlichen Umverteilungsgewinne hinaus zu einer relativ hohen Belastung der Bürger führt und damit eine gefahrenträchtige Strategie darstellt, stellt sich ohne eine Budgetgrenze auch. Allerdings ist in diesem Fall eher die Frage, ob eine Erhöhung des Budgets von den Steuerzahlern sanktioniert wird oder nicht. Es geht darum, den Wählern die letzte Mark an Staatsausgaben wertvoller erscheinen zu lassen als eine Mark an Steuerzahlungen.

Diese Option stellt sich im Fall der Budgetbeschränkung nicht mehr. Eine Erhöhung der Ausgaben ist durch die verfassungsmäßige Begrenzung ausgeschlossen. Je größer jedoch die Ineffizienz der Umverteilungsmaßnahme ist, umso geringer ist der Anteil der von der Interessengruppe realisierten Gewinne am jeweiligen Budgetvolumen. Dieser verringerte Umverteilungsgewinn kann nicht mehr durch einen erhöhten Budgetumfang ausgeglichen werden. Jede Interessengruppe wird sich somit bemühen, zu möglichst effizienten, d.h. Wenig ausgabenintensiven Maßnahmen zur Erzielung von Verteilungsgewinnen zu greifen.1)

Im wesentichen dirfte daher von der Einfiurung einer Budgetgrenze eine Verringerung der situationsbezogenen Ineffizienz des States zu erwarten sein.

Dazu ist aber folgendes zu bemerken: Innerhalb der Interessengruppentheorie des Staats kann im Regelfall nicht geklärt werden, wieso es uberhaupt zu einer Bereitstellung öffentiicher Güter kommt. Im wesentiichen konkurrieren die einzelnen Interessengruppen ja um Verteilungsgewinne durch den statilichen Haushalt, d.h. um private Guiter.

i) vgl. dazu BECKER (1983) S. $381 \mathrm{ff}$. 
$D a B$ es uiberhaupt zu einer Versorgung mit öffentlichen Gütern kommt, kann dann unter drei Gesichtspunkten begründet werden:

1. Der Konsum des öffentlichen Gutes kann selbst das politische $z i e l$ der Interessengruppe sein, d.h. die Mitglieder der Interessengruppe versuchen nicht, Gewinne durch eine erhöhte Versorgung mit privaten Gütern zu erreichen, sondern durch den erhöhten Konsum an öffentlichen Gütern. Angenommen, die Höhe der statlichen Ausgaben ist unabhängig davon, ob sie privaten Transfers oder der Bereitstellung rein öffentlicher Güter dienen. Dann wird eine Interessengruppe sich immer dann für die Produktion eines öffentlichen Gutes einsetzen, wenn dadurch die Mitglieder einen höheren Nettogewinn erzielen als durch reine Transferzahlungen.

Damit kann die betrachtete Interessengruppe quasi als club angesehen werden, der $z$ wei voneinander unterschiedliche Funktionen erfiult. Einmal tritt sie als eine Organisation hervor, die ihren Mitgliedern Verteilungsgewinne über den Staatshaushalt zuteilen will, d.h. als typische einflußnehmende Institution. Zum zweiten stellt sie einen privaten Club zur Bereitstellung öffentlicher Güter dar. Diese beiden Funktionen könnten im Prinzip auch von zwei verschiedenen Organisationen übernommen werden.

Allerdings können diese völlig unterschiedlichen Funktionen möglicherweise nur deshalb wahrgenommen werden, da ein gemeinsamer organisatorischer Rahmen vorhanden ist. OLSON (1965) wies bereits darauf hin, daß eine Organisation nur dann erfolgreich operieren $k a n n$, wenn sie neben der Versorgung mit öffentlichen Gütern auch Dienstleistungen anbietet, bei denen der AusschluB von Nichtmitgliedern möglich ist. In jedem anderen Fall düfte ab 
einer bestimmten Größenordnung das Schwarzfahrerverhalten potentieller NutznieBer eine effiziente Organisation und damit Interessenvertretung verhindern.1)

Sobald die Organisation zwei separate Aufgaben zu erfiillen hat, nämlich die Bereitstellung öffentlicher Güter und die EinfluBnahme auf den politischen ProzeB zum Erwerb von Einkommen, ist diese Voraussetzung erfiilt: Durch den Erwerb von Einkommen aus dem politischen UmverteilungsprozeB versorgt die Organisation jeden einzelnen mit einem mit AusschluBcharakter versehenen privaten Gut - damit ergibt sich ein Anreiz, der Organisation beizutreten. Zum zweiten kann dann die Organisation sich um die Bereitstellung kollektiver Gïter kümmern, die ihren Mitgliedern zur Verfügung gestellt werden. Die Problematik einer optimalen Versorgung mit öffentlichen Gütern ist damit allerdings nicht aufgehoben, nur verlagert sich die Entscheidung, welcher Anteil des im politischen Bereich bezogenen Einkommens nun fijr die Bereitstellung öffentlicher Güter oder für die Auszahlung privater Transfers an die Mitglieder der Interessengruppe verwandt wird, auf die Organisation selbst und muB dort gelöst werden. Das Ergebnis hängt dann ab von den innerhalb der Interessengruppe herrschenden Entscheidungsstrukturen.

Immerhin kann es aufgrund der Gruppenaktivität zur Bereitstellung von öffentlichen Gitern kommen, die von den Mitgliedern dieser Gruppe nachgefragt werden. Dabei können die beiden Aktivitäten - Bereitstellung öffentlicher Güter und Kampf um politisches Einkommen -, obwohl funktionell

1) Natürlich können auch öffentliche Güter durchaus die Eigenschaft der AusschlieBbarkeit aufweisen. In diesem Fall stellt sich das Problem nicht in der Schärfe, dennoch ergibt sich ein Problem hinsichtlich der Bestimmung des optimalen Beitrags der einzelnen Mitglieder, das nur dann trivial ist, wenn alle dieselben Präferenzen haben oder diese bekannt sind. 
getrennt, uno actu verfolgt werden. Statt Zahlungen an die Interessengruppe $z u$ leisten, die ihrerseits für die Bereitstellung der öffentlichen Güter sorgt, kann der Stat letzteres auch direkt selbst übernehmen, bzw. die Zahlungen werden als zweckgebundene Zuweisungen ausgezeichnet.

Die Versorgung mit öffentlichen Gütern ergibt sich somit als Produkt privater Aktivität und könnte (abgesehen von den spezifischen AusschluBproblemen) durchaus ohne die Existenz eines Zentralstats zustande kommen.

Allerdings finden sich alle angesprochenen Probleme, die für den EntscheidungsprozeB auf staatlicher Ebene gelten, ebenso beim EntscheidungsprozeB innerhalb von Organisationen; eine optimale Versorgung mit öffentlichen Gütern im Sinne der Paretoeffizienz ist nicht zu erwarten. Immerhin weisen organisierte Interessenverbände im Gegensatz zu staatlichen Organisationen den Vorteil auf, daB in der Regel kein Bürger zu einer Mitgliedschaft in dieser Organisation gezwungen ist bzw. der Austritt aus dem Kollektiv leichter ist als der Austritt aus dem Staatsverband.

2. Neben der Bereitstellung von öffentlichen Gütern auf Initiative von Interessengruppen kann diese auch auf Initiative der Politiker erfolgen, um den Präferenzen von (unorganisierten) Bürgergruppen, deren Wahlentscheidung einen großen EinfluB hat, zu entsprechen. Es handelt sich bei diesen Gruppen quasi um "latente Interessengruppen",1) die zwar nicht durch eine Organisation vertreten werden, deren Interessen aber auch ohne verbandsmäßige Vertretung leicht identifizierbar sind und die über hinreichende Informationen über die Resultate politischer Entscheidun-

1) WAGNER (1966) 
gen verfügen, um eine rationale Wahlentscheidung $z$ wischen verschiedenen politischen Vorschläge zu treffen. Im Idealfall der vollkommenen politischen Konkurrenz ist dies ja Grundlage einer optimalen Statstätigkeit.

3. Eine letzte Möglichkeit, wie eine Versorgung mit öffentlichen Gütern zustande kommen kann, liegt darin, daß diese Nebenprodukt der Konkurrenz der Interessengruppen um private Einkommen sein kann.1)

In Abschnitt 3.3.4.3 wurde gezeigt, daB eine Ausdehnung des Budgets ibber die Höhe der als Verteilungsgewinne zur Auszahlung kommenden Beträge hinaus aus Sicht der Interessengruppe durchaus rational sein kann, um den Informationsgehalt dieser Maßnahmen und damit die politischen Widerstände möglichst gering zu halten. Die Menge an Ressourcen, die dabei ubber den reinen Umverteilungszahlungen liegt, wurde als gesellschaftliche Verschwendung bezeichnet.

Dies muB aber nicht so sein. Verschwendung stellen sie primär nur dar aus der Sicht der Interessengruppen, die dadurch anderen Mitgliedern der Gesellschaft Mittel entziehen, die sie selbst nicht als Verteilungsgewinne einstecken können. Die "verschwendeten" Ressourcen können dabei durchaus zu positiven Ergebnissen für die anderen Mitglieder der Gesellschaft verwandt werden.

Nehmen wir als Beispiel wieder den zitierten Baunternehmer. Seine Lobbytätigkeit für Aufträge mögen als einzigen Grund die Erzielung privater Gewinne haben, aus seiner Sicht sind alle statichen Ausgaben, die sein eigenes Einkommen nicht erhöhen, als Verschwendung anzusehen. Daraus folgt nicht, daß die Herstellung

i) $v$ gl. AUSTER (1974), ARANSON/ORDESHOOK (1981) S. 163 
öffentlicher Gebäude für die anderen Mitglieder der Gesellschaft nicht doch von Nutzen sein kann. Den Bauausgaben kann durchaus eine positive $Z$ ahlungsbereitschaft der Staatsbürger gegenüber stehen, sie können durchaus sinnvoll sein. Dies ist umso wahrscheinlicher, als eine widerstandsvermindernde Strategie der Baulobby insbesondere dann Erfolg haben durfte, wenn neben den Umverteilungsgewinnen für die Mitglieder der Interessengruppe auch ein positiver Nutzenzuwachs für die übrigen Mitglieder der Gesellschaft aus den staatlichen Ausgaben erwächst.

Dabei besteht sogar die Möglichkeit, daß diese staatlichen Ausgaben ohne aktive EinfluBnahme der jeweiligen Lobby gar nicht zustande gekommen wären - die Bereitstellung öffentlicher Guiter ist sozusagen ein externer Effekt der Tätigkeit von Interessengruppen, die an eigenen, privaten Gewinnen aus dem politischen ProzeB interessiert sind.

Dabei dirfte das Ergebnis des politischen Prozesses allerdings nicht eine optimale Versorgung mit öffentlichen Gütern im Sinne des Pareto-0ptimums sein. Das Kalkül, das der Bestimmung des Umfangs der öffentlichen Leistungen zugrunde liegt, ist ja nicht eine optimale Versorgung im Sinne eines Ausgleichs der Grenzrate der Transformation und der Summe der Grenzraten der Substitution, sondern die Produktion öffentlicher Giuter orientiert sich in erster Linie daran, inwieweit die einzelnen Interessengruppen auf diese Weise noch eine Erhöhung ihrer privaten Einkommen durchsetzen können. Ein Zusammenfallen von sozialem und privatem Optimum wäre reiner Zufall.1)

i) Und ist unter bestimmten technologischen Bedingungen gar nicht denkbar. Handelt es sich bei der Produktion des öffentlichen Gutes um eine linear homogene Produktionsfunktion und stehen die Anbieter des öffentlichen Gutes in vollkommener Konkurrenz zueinander, so wird bei einer optimalen Versorgung gerade Kostendeckung erreicht und eine Erhöhung der Nachfrage nach öffentlichen Giutern via EinfluBnahme der Produzenten hätte keinen Sinn. Eine erfolgreiche Lobbbytätigkeit setzt 
Die Einführung von Budgetbegrenzungen wird damit aber zu einer fragwiurdigen Methode, um statswirtschaftiche Ineffizienzen abzubauen. Der wesentliche Effekt der Budgetbeschränkung ist $j a, d a B$ die "ausgabenintensiven" Umverteilungsmaßnahmen zurückgehen. Diese sind ja mit sehr hohen statichen Aufwendungen verbunden, die aus der Sicht der Interessengruppe Verschwendung darstellen, d.h. ihr Einkommen nicht erhöhen. Stattdessen werden eher MaBnahmen getroffen werden, bei denen der Anteil der umverteilend wirkenden Ausgaben am Gesamtbudget relativ hoch ist. Wenn aber gerade das, was aus Sicht der Interessengruppen nur Verschwendung darstellt, für die nichtorganisierten Bürger nutzenstiftend ist, so führt eine Budgetbeschränkung zu folgendem perversen Effekt: Die aus allgemeiner Sicht relativ effizienten Ausgaben werden aufgrund der Budgetbeschränkung zurückgehen, während die "umverteilungsintensiven", d.h. die Ausgaben, bei denen der Anteil der spezifischen Begünstigungen relativ hoch sind, wenig tangiert werden oder sogar noch steigen.2) Eine Budgetbeschränkung könnte somit sogar den Grad der Ineffizienz des öffentlichen Haushalts noch verstärken, d.h. zu einem höheren Staatsversagen führen.

somit bestimmte technologische Bedingungen ( 2 . B. steigende Grenzkosten) oder eine bestimmte Marktform (Monopol) voraus, wenigstens, solange damit bleibende Gewinne aus dem politischen ProzeB gezogen werden können. Vgl. ROSE-ACKERMANN (1975) S. 188

2) Wenn die Interessengruppen wegen der Budgetbeschränkung ihre Ressourcen zum Gewinn von spezifischen Beginstigungen von den relativ ausgabenintensiven zu den umverteilungsintensiven umschichten. 
Ähnliches gilt, wenn wir den Konflikt latente Interessengruppen - existierende Interessengruppen betrachten, der durch die Budgetbeschränkung auftritt.

Als möglicher Grund für die Bereitstellung öffentlicher Güter wurde ja die Berïcksichtigung der Interessen von nichtorganisierten Wahlbürgern durch die Politiker genannt. In dem Augenblick, da eine Budgetbeschränkung eingeführt wird, steht der Politiker vor der Wahl, entweder die Interessen der nichtorganisierten Wähler zu berücksichtigen und die Bereitstellung öffentlicher Güter zu deren Gunsten auf Kosten der den spezifischen Interessengruppen zugute kommenden Ausgaben vorzunehmen, oder das Gegenteil zu tun. Dieser Konflikt besteht ohne die Budgetbeschränkung nicht in dem Maße, da ja eine Ausgabenerhöhung zu Gunsten der einen Gruppe nicht unbedingt eine Reduktion der Ausgaben, die den anderen zugute kommt, impliziert.

War vorher aus Sicht der Politiker ein optimales Budget dann gegeben, wenn der Grenzgewinn an Stimmen durch eine Erhöhung der Ausgaben jedweder Kategorie gleich Null war, so ist im Falle einer vorgegebenen Budgetgrenze für den Politiker nur noch die Struktur der Ausgaben ein Handlungsparameter, d.h. er wird die Ausgabenstruktur wählen, bei der der Grenzgewinn an Stimmen für jede Ausgabenart gleich ist. Anders ausgedrückt, es werden zuerst die Ausgaben gekürzt, bei denen der marginale Verlust an Stimmen relativ gering ist. Das können aber auch die öffentlichen Leistungen sein, die rein öffentliche Guiter im herkömmlichen Sinn darstellen und deren Bereitstellung eigentlich der effiziente Teil der Statstätigkeit war. Im Extremfall kann eine Budgetgrenze dazu führen, daß Pareto-Verbesserungen allein deswegen nicht vorgenommen werden, da dies nur über eine Erhöhung staticher Ausgaben möglich wäre und durch die Budgetbegrenzung dies 
nur auf Kosten anderer Ausgaben durchgesetzt werden kann. Mit anderen Worten, ein nicht-existierender Interessenkonflikt wird durch die Budgetbeschränkung erst aufgebaut.

Auf Grund von A-priori-oberlegungen läBt sich also nicht eindeutig folgern, daB eine Budgetbeschränkung zu einer höheren Effizienz im staatlichen Sektor führt, sondern auch das Gegenteil kann der Fall sein. Damit entfält aber das gewichtigste Argument für eine verfassungsmäBige Begrenzung der Ausgaben, das ja gerade darauf beruhte, daB sie als A-priori-Restriktion, also ohne Betrachtung der konkreten Folgen, zu einer Verbesserung des Ergebnisses staticher Entscheidungsprozesse führen sollte. Eben unter diesem Gesichtspunkt läßt sich der Sinn von Budgetbegrenzungen nicht eindeutig beweisen.

\subsubsection{Die Problematik von Ausweichmöglichkeiten}

Neben der Möglichkeit, daB der Grad der Effizienz der staatlichen Ausgaben durch die Budgetbeschränkung vermindert statt erhöht wird, kann gegen eine Ausgabengrenze eingewandt werden, daB Staatsausgaben nicht die einzige Methode sind, durch die Interessengruppen eigene Einkommensvorteile erzielen können. Neben den Ausgaben können Einkommmensvorteile auch dadurch erreicht werden, indem den Interessengruppen bestimmte spezifische Steuervorteile eingeräumt werden. Steuererleichterungen werden aber durch eine Ausgabenbeschränkung nicht betroffen, sie führen ja eher zu einer Verringerung des Budgetumfangs und gehen damit mit dieser spezifischen institutionellen Begrenzung konform.

Eine Ausgabengrenze würde dabei dazu führen, daß es zu einer Verlagerung des Wettbewerbs um politisches Einkommen von den Statsausgaben weg zu spezifischen Steuererleich- 
terungen hin käme. Effizienzvorteile dieser Verlagerung sind aber nicht ohne weiteres erkennbar. Steuerliche Sondervorteile können genauso effizienzmindernd sein wie statliche Ausgaben.

Dem Wettbewerb um politisches Einkommen wird durch die Einführung von Budgetgrenzen kein Ende gemacht, es kommt zu einer Verlagerung der Ressourcen, die vorher zur Erzielung von Verteilungsgewinnen durch öffentliche Ausgaben eingesetzt wurden, in die Konkurrenz um Steuervorteile.

Der Zusammenhang ist allerdings etwas komplexer und soll in einem Modell geklärt werden, in dem einem Wirtschafts subjekt drei Möglichkeiten des Ressourceneinsatzes zur Auswahl stehen: Einmal können die Ressourcen zur produktiven $Z$ wecken eingesetzt werden, um dadurch Einkommen $z u$ erzielen. Zum zweiten können sie in den politischen ProzeB gesteckt werden, und $z$ war einerseits, um Einkommensvorteile aus öffentlichen Ausgaben zu erhalten, und andererseits, um die Steuerbelastungen zu verringern, d.h. Einkommen durch spezifische Steuererleichterungen zu erreichen. Vermiedene Steuerzahlungen können ja als Einkommen angesehen werden. Zur Vereinfachung sollen die in den politischen ProzeB eingesetzten Ressourcen als verschwendet betrachtet werden. Im Gleichgewicht muB dann der Grenzertrag für jede der drei alternativen Verwendungsmöglichkeiten von Ressourcen gleich sein.

Nach Einführung einer Budgetgrenze soll der Ertrag der Ressourcen, die zur Produktion von politischem Einkommen durch Staatsausgaben eingesetzt werden, sinken. Es kommt zu einer Reallokation der Ressourcen, die Höhe der zur produktiven Verwendung wie zur Einflußnahme auf steuerliche Gegebenheiten eingesetzten Ressourcen wird steigen. Damit wird zumindest ein Teil der sonst in die (gesamt- 
wirtschaftich) unproduktive politische Einflußnahme investierten Ressourcen einer produktiven Verwendung zugeführt.

Das Problem ist nur, daß die Verlagerung der Ressourcen nicht nur von einem Individuum vorgenommen wird, sondern von allen. Nun duirfte im allgemeinen die Ertragsrate des Einsatzes von Ressourcen in den politischen ProzeB nicht unabhängig sein von der Menge an Ressourcen, die andere Interessengruppen zur Einflußnahme aufwenden. Die (Pseudo-)Produktionsfunktion für Einkommen aus steuerlichen Maßnahmen wird sich verändern. A priori kann dabei keine eindeutige Aussage über die Wirkung dieser Veränderung getroffen werden. Die Menge der zur politischen EinfluBnahme eingesetzten Ressourcen kann sowohl steigen als auch fallen, wie in Abschnitt 3.3 .5 .1 bereits beschrieben wurde.

Konsequenterweise verlangen daher zahlreiche Autoren die Einbeziehung von Steuervergünstigungen in eine Ausgabengrenze.1) Allerdings dürfte es wohl kaum im einzelnen zu entscheiden sein, welche Maßnahme als Steuervergünstigung anzusehen sind und welche nicht. Bereits bei der Definition der Bemessungsgrundlage gibt es genügend Möglichkeiten, eine Vergünstigung zu erlangen. Dabei wird nicht $k l a r$, inwieweit bestimmte, in der Bemessungsgrundlage nicht berücksichtigte Tatbestände bereits als Steuervergünstigung anzusehen sind. Ebenso durfte die Behandlung progressiver Tarife Schwierigkeiten bereiten, da auf gewisse Art jede Abweichung vom Spitzensteuersatz als Steuervergünstigung bezeichnet werden kann.

i) vgl. WILDAVSKY (1980) 
Die Abgrenzungsschwierigkeiten ergeben sich im Vergleich zu der Definition von Staatsausgaben vor allem deswegen, weil bei Steuervergüntigungen hypothetische $Z$ ahiungs ströme betrachtet werden. Je nachdem, welche Abgrenzung man dabei vornimmt, kann es zu erheblichen Differenzen kommen. Wenn aber solche Differenzen möglich sind, dann kann eine verfassungsmäßige Begrenzung kaum Erfolge zeigen. Der Sinn einer Verfassungsregel ist $j a, d a B$ der Rahmen vorgegeben wird, in dem spätere Entscheidungen $z u$ treffen sind. Werden konkrete Definitionsprobleme zum Gegenstand von Entscheidungen auf postkonstitutioneller Ebene, verliert die Budgetbegrenzung diesen Charakter.

Eine Alternative zum EinschluB von Steuererleichterungen in eine Staatsausgabengrenze wäre die zusätzliche Begrenzung des Steueraufkommens nach unten. Damit wäre ein ähnlicher Effekt bezweckt wie durch eine Staatsausgabengrenze - eine Einschränkung der Möglichkeiten, durch spezifische Steuererleichterungen Vorteile zu erlangen. Aber abgesehen von den bereits hinsichtlich der Wirksamkeit von statsausgabengrenzen kritisch angemerkten Punkten wirde eine solche Regelung letztendlich auf eine Festlegung der Statsquote hinauslaufen, womit eine relativ starke Inflexibilität verbunden wäre.

Schlieblich: Spezifische Steuervorteile schlagen sich in Höhe und Struktur des Budgets nieder, damit könnten Budgetbegrenzungen noch begründet werden; völlig versagen müssen aber budgetäre Maßnahmen gegenüber politischer Einflußnahme, die Einkommensvorteile zu erzielen versucht, ohne daB der Staatshaushalt dabei uberhaupt berührt wird. Der Staat nimmt ja nicht nur durch Zahlungen und Steuereinnahmen Einfluß auf wirtschaftliche Gegebenheiten, auch nichtbudgetäre Maßnahmen können zu Einkommensvorteilen/-nachteilen umschlagen. Zu denken ist dabei an Möglichkeiten, bestimmte Märkte zu regulieren, z.B. durch 
statlich geschaffene Zutrittsbeschränkungen, durch Preisregelungen und -garantien für privat hergestellte und auch konsumierte Güter (Mietpreisbindungen, gesetzliche Tarifbestimmungen, Mindestlöhne usw.). Auch die Aufnahme von Ressourcen durch den Staat muß nicht immer ein entsprechendes budgetäres Äquivalent finden: Beschlagnahmungen, die gesetzliche Verpflichtung von Privaten zur Mitwirkung an bestimmten staatlichen Leistungen (wie zum Beispiel Wehrpflicht) und anderes sind ebenso Methoden, Ressourcen dem privaten Sektor zu entziehen und auf andere Weise wieder zur Verfügung zu stellen.

Die Effekte statlicher Eingriffe zur Regulierung von Märkten können durchaus den Effekten von staatlichen Ausgaben und Steuern gleich kommen, 1) auch hier können Umverteilungsgewinne/-verluste entstehen und Aktivitäten auf diesem Feld dürften denselben Kalkülen unterliegen wie die Konkurrenz um Vorteile aus budgetären Aktivitäten des States. Gegen solche Maßnahmen ist eine Budgetgrenze unwirksam.

Anzunehmen ist jedoch, daß durch die Einführung einer Budgetbeschränkung die Aktivität der Interessengruppen sich stärker auf solche Gebiete verlagern wird, wo durch staatliche Maßnahmen, ohne daß das Budget berührt wird, Umverteilungsgewinne realisiert werden können. Die Nachfrage nach Regulierung bzw. die Höhe der zur Herbeiführung nichtbudgetärer staatlicher Maßnahmen eingesetzten Ressourcen wird steigen. Die damit verbundene Ineffizienz kann größer sein als die, die vor Einführung einer Budgetbeschränkung existierte.

1) $\mathrm{Vgl.POSNER}(1971)$ 
Zudem: Wenn durch eine Budgetbeschränkung alle Vorgänge, die auBerhalb des Haushalts bleiben, nicht beriurt werden, liegt es nahe, bestimmte Manipulationen an der Definition des Begriffs Staatsausgaben vorzunehmen und diese aus dem eigentlichen Haushalt auszulagern. So eindeutig ist der Begriff öffentliche Ausgaben nicht, als daB nicht durch reine Umdefinition eine "Flucht aus dem Budget" möglich wäre.1) Zwar gilt nach dem Budgetprinzip der Vollständigkeit, daB alle staatlichen Einnahmen und Ausgaben unsaldiert, also zu Bruttowerten, in den Haushalt einbezogen werden sollen, faktisch wird dieses Prinzip aber oft durchbrochen. Dazu zählt zum Beispiel die Tatsache, daß Bundesbetriebe in der Regel im Budget nur mit ihren $A b l i e f e r u n g e n ~ b z w$. Zuführungen aufgeführt werden.

Damit besteht aber die Möglichkeit einer Staatsausgabenerhöhung über die Budgetgrenze hinaus, indem eigene, nur mit dem Finanzierungssaldo in der Haushaltsrechnung erfaßte Körperschaften gegründet werden, denen eigene Finanzierungsquellen zugewiesen werden, wodurch die Budgetbeschränkung unterlaufen wird. Zwar ist theoretisch eine vollständige Erfassung dieser Körperschaften im Budget denkbar, aber hier ergeben sich doch Abgrenzungsprobleme und Ausweichmöglichkeiten. Wie zum Beispiel sind Ausgaben zu gewichten, die von Organisationen getätigt werden, an denen der Staat nur zu einem gewissen Prozentsatz beteiligt ist? Wie sind Institutionen zu werten, die zwar vom Charakter her private organisationen sind, aber in einen öffentlich rechtlichen Rahmen gestelit werden? Welche Behandlung erfahren Zahlungen $z$ wischen verschiedenen statichen Stellen?

1) Dazu und zum folgenden SENF (1976) S. $393 \mathrm{ff}$. 
Natürlich ließe sich für alle angesprochenen Punkte eine Lösung finden, die Gefahr besteht aber, daB durch die Erfindung neuer institutioneller Regelungen diese jeweiligen Bestimmungen unterlaufen werden können. Als Beispiel mag die Entwicklung in den USA zitiert werden, wo dem "Government Corporation Control Act" von 1945, der das Einnahme- und Ausgabegebaren von Statsunternehmen denselben Regeln unterwarf wie normale Staatseinnahmen/-ausgaben, die Entwicklung einer Fülle von staatlichen Organiationsformen folgte, die allein dem $Z$ weck dienten, diese Prozedur zu umgehen.1)

Die Möglichkeit der "Flucht aus dem Budget" führt aber dazu, daß die Entscheidungen, ob die Ausgaben bestimmter Institutionen in die Budgetgrenze einzubeziehen sind oder nicht, wiederum auf post-konstitutioneller Ebene getroffen werden müsen und somit der Charakter der Budgetbegrenzungen als Verfassungsregel in Frage gestellt ist.

Zudem hat eine allein durch die Einführung der Budgetbegrenzung provozierte Auslagerung von Ausgaben aus dem eigentlichen Haushalt zur Folge, daB der politische EntscheidungsprozeB noch undurchsichtiger und gegebenenfalls noch ineffizienter wird. Der Haushaltsplan hat ja auch die Funktion, dem Parlament ein Mittel der Kontrolle in die Hand zu geben -Ausgliederungen aus dem Haushalt, die einzig und allein aus dem Motiv der Umgehung von Budgetbegrenzungen getätigt werden, verhindern oder erschweren zumindest die Erfüllung dieser Aufgabe.

Darüber hinaus wird die Entscheidung, ob eine bestimmte Aktivität aus dem Budget zu finanzieren sei, oder einer "ausgelagerten" Institution übertragen wird, die vorher unter reinen Effizienzgesichtspunkten getroffen werden

i) vgl. SENF (1976) S. 397 
konnte, jetzt systematisch zugunsten einer Organisationsform, die nicht durch die Budgetbegrenzung erfaBt wird, verzerrt. Dies wird sicherlich nicht zu einer Erhöhung der Effizienz der Statswirtschaft beitragen.

Die amerikanischen Erfahrungen illustrieren deutlich die Möglichkeiten, die in einer Verlagerung von Staatsausgaben aus dem Budget heraus liegen. In Staten, in denen eine Einführung von Steuer- und Ausgabengrenzen durchgesetzt wurde, expandieren die "Off-Budget"-Aktivitäten sprunghaft.1)

Eine sinnvolle Ausgabengrenze setzt somit nicht nur eine genaue Definition dessen, was unter dem Begriff "Staatsausgaben" $z u$ verstehen ist, voraus, sondern auch, daB Umgehungsmöglichkeiten, die von dieser Bestimmung nicht erfaßt werden, zumindest nur in geringem Maße möglich sind. Diese Bedingung ist jedoch nicht erfiillt.

i) $\vee$ I. BENNET/DILORENZO (1982) 


\section{4. $\operatorname{sen} 7 u B$}

Insgesamt lassen sich keine eindeutigen Folgerungen über die Vorteilhaftigkeit einer Einführung von verfassungsmäBigen Budgetgrenzen, speziell Ausgabengrenzen ziehen. Auf der Angebotsseite ergab sich zwar, $d a B$ eine Budgetbegrenzung $z u$ einer Verbesserung der Effizienz der öffentlichen Leistungserstellung führt, wobei der Mechanismus im wesentichen auf der Herbeiführung einer konkurrenzähnlichen Situation innerhalb des öffentlichen Sektors beruht. Allerdings wurde nicht untersucht, inwieweit eine solche Wettbewerbsbeziehung nicht auch durch andere, nichtbudgetäre Maßnahmen erreicht werden kann, z.B. durch ein monetäres Anreizsystem für die Bürokratie oder ähnliches.

Während jedoch die Herstellung von wettbewerbsähnlichen Zuständen, soweit allein die Angebotsseite betrachtet wird, noch positiv zu bewerten ist, sind die Ergebnisse, die Nachfrageseite betreffend, ambivalent. Auch hier führt eine Einführung von Budgetgrenzen dazu, daß der Versuch, über den politischen ProzeB Einkommensvorteile zu realisieren, einem verstärkten Konkurrenzdruck unterliegt. Anders aber als im privaten ProduktionsprozeB, wo die durch den Wettbewerb induzierten Qualitäts - und Verfahrensinnovationen normalerweise auch gesellschaftich positiv zu bewerten sind, gilt dies für den politischen ProzeB nicht. Die Konkurrenz um politisches Einkommen ist, vom kollektiven Standpunkt aus, unproduktiv; Innovationen im ProzeB des Wettbewerbs um Transfers führen also nicht $z$ wangsläufig zu einer Verbesserung der gesellschaftlichen Situation, sondern können auch kontraproduktive Wirkungen 
zeigen. Das gilt auch für die Bürokratie als Anbieter öffentlicher Leistungen, soweit sie EinfluB auf die Nachfrage nimmt.

Aufgrund von A-priori-oberlegungen läBt sich also nicht eindeutig sagen, ob eine budgetäre Beschränkung zu einer Verminderung oder VergröBerung des "Staatsversagens" führt: Die Ergebnisse sind ambivalent. Damit fällt aber auch das in Abschnitt 2.2 entwickelte Argument für eine verfassungmäBige Verankerung einer Budgetgrenze fort: Eine eindeutige Aussage, ob es zu einer Verbesserung oder Verschlechterung kommt, setzt eine Analyse der konkreten Folgen der Einführung der Budgetbegrenzung voraus. Dann aber sind auch Gewinner und Verlierer dieser Maßnahme identifizierbar. Damit wird das Zustandekommen einer verfassungsmäBigen Einigung auf eine konstitutionelle Budgetbegrenzung erschwert. Einstimmigkeit könnte nur noch dann erzielt werden, wenn wirklich alle Beteiligen mit Sicherheit gewinnen würden, die Budgetbeschränkung also eine Paretoverbesserung nach sich zöge. Dies ist jedoch kaum zu erwarten.

Anders ausgedrückt: $O b$ eine verfassungsmäBig verankerte Budgetbegrenzung zu einer Erhöhung der staatswirtschaftlichen Effizienz führt, ist eine empirisch zu lösende Frage, die nicht von der Theorie allein beantwortet werden kann. Damit ist nicht gesagt, daB eine solche konstitutionelle Regelung nicht wünschenswert wäre; allerdings ist nicht zu erwarten, $d a B$ ein derartiger Vorschlag auch unbedingt auf ungeteilten Beifall aller am politischen ProzeB Beteiligten treffen wird. Die Chancen, daß eine solche Regel durchgesetzt werden $k a n n$, sind nicht allzuhoch zu veranschiagen. 
Zudem wurde nicht untersucht, welche anderen Möglichkeiten einer Erhöhung der staatswirtschaftlichen Effizienz bestehen und inwieweit die Anwendung solcher Instrumente nicht einer globalen Maßnahme wie der Festschreibung der Staatsquote vorzuziehen sind. VerfahrensmäBige Änderungen ( $z$. B. die Integration von Einnahme- und Ausgabeentscheidungen 1)) mögen dabei wesentlich positivere Effekte nach sich ziehen als quantitative Beschränkungen.

Letztendlich stellt sich das Problem des Staatsversagens im wesentlichen als ein Informationsproblem dar: Nur wenn unvollkommene Information vorhanden ist, d.h. der Prozeß der Informationsgewinnung und -verarbeitung $n i c h t$ kostenlos ist, können sich spezifische Interessen gegen Mehrheitsinteressen durchsetzen bzw. Wird die Beteiligung am politischen ProzeB zu einer kostspieligen Angelegenheit. In diesem Sinn ist jede Maßnahme zu begrüBen, die die Informationsbeschaffungs - und -verarbeitungskosten senkt. Budgetgrenzen zielen auf indirekte Weise dahin: Durch die aufgrund der Budgetbeschränkung eingeführte bzw. verschärfte Konkurrenzsituation der Anbieter und Nachfrager öffentlicher Leistungen soll dieser Informationsstand verbessert werden. $D a B$ Budgetbegrenzungen diesem Anspruch nur in begrenztem Rahmen gerecht werden können, sollte in dieser Arbeit gezeigt werden.

i) vgl. MUSGRAVE (1981) S. $113 \mathrm{ff}$. 


\section{Literaturverzeichnis}

AARON, H./MCGUIRE, M. (1970): Public Goods and Income Distribution, in: Econometrica 38,5 . $907-921$

ALCHIAN, A. (1965): The Basis of Some Recent Advances in the Theory of Management of the Firm, in: Journal of Industrial Economics 14 , S. $30-41$

AMACHER, R./TOLLISON, R. /WILLETT, D. (1975): Budget Size in a Democracy: a Review of Arguments, in: Public Finance Quarterly 3. S. $99-121$

ARANSON, P./FERROR, T./SASSONE, P. (1978): Pigouvian Politics in Closed Behavourial Systems, in: Public Choice $33, \mathrm{~S}$. $5-26$

ARANSON, P./ORDESHOOK, P. (1981): Alternative Theories of the Growth of Government and their Implications for Constitutional Tax and Spending Limits, in: LADD, H./TIDEMAN, T. (eds.): Tax and Expenditure Limitations, Washington D.C., S. $143-175$

ARROW, K. (1962): Collective Choice and Individual Values, 2. Auflage, New Haven/London

AUSTER, R. (1974): The GPITPC and Institutional Entropy, in: Public Choice 19, S. 77-83

BARRO, R. (1973): The Control of Politicians: An Economic Model, in: Public Choice 14, S. $19-42$

BARTLETT, R. (1973): Economic Foundations of Political Power, New York

BATOR, F. (1958): The Anatomy of Market Failure, in: Quarterly Journal of Economics 72,5 . $351-379$

BAUM, Th. (1982): Statsverschuldung und Stabilisierungspolitik in der Demokratie, Frankfurt/Bern 
BECKER, G. (1983): A Theory of Competition Among Pressure Groups for Political Influence, in: Quarterly Journal of Economics 98 , S. $371-400$

BENNET, J./DILORENZO, R. (1982): Off-Budget Activities of Local Government: The Bane of the Tax Revolt, in: Public Choice 39, $S$. $333-342$

BEN-ZION, U./EYLAU, Z. (1974): On Money, Votes and Policy in a Democratic Society, in: Public Choice 17, S. 1-10

BERNHOLZ, P. (1969): Einige Bemerkungen zur Theorie des Einflusses der Verbände auf die politische willensbildung in der Demokratie, in: Kyklos 22, S. $276-288$

BERNHOLZ, P. (1974): Logrolling, Arrow Paradox and Decision Rules: a Generalization, in: Kyk los 27 , S. $49-62$

BERNHOLZ, P. (1975): Grundlagen der Politischen okonomie, 2. Band, Tübingen

BERNHOLZ, P. (1977): Dominant Interest Groups and Powerless Parties, in: Kyklos $30, \mathrm{~S}$. $411-420$

v. BEYME, K. (1980): Interessengruppen in der Bundesrepublik Deutschland, 5. Auflage, München

BLACK, D. (1958): The Theory of Committees and Elections, Cambridge

BLANKART, Ch. (1975): Zur ökonomischen Theorie der Bürokratie, in: Public Finance $30, \mathrm{~S}$. $166-175$

BORCHERDING, Th./BUSH, W./SPANN, R. (1977): The Effects of Public Spending on the Divisibility of Public output in Consumption, Bureaucratic Power, and the Size of the Tax-Sharing Group, in: BORCHERDING, Th. (ed.): Budgets and Bureaucrats: The Sources of Government Growth, Durham, S. 211-228

BRENNAN, G./BUCHANAN, J. (1977): Towards a Tax Constitution for Leviathan, in: Journal of Public Economics 8, S. 255-274 
BRENNAN, G./BUCHANAN, J. (1978): Tax Instruments as Constraints on the Disposition of Public Revenues, in: Journal of Public Economics 9, S. 301-318

BRENNAN, G./BUCHANAN, J. (1979): The Logic of Tax Limits: Alternative Constitutional Constraints on the Power to Tax, in: National Tax Journal 32 , Suppl., S. $11-22$

BRENNAN, G./BUCHANAN, J. (1980): The Power to Tax, Cambridge

BRETON, A. (1974): The Economic Theory of Representative Government, Chicago

BRETON, A./WINTROBE, R. (1975): The Equilibrium Size of a Budget Maximizing Bureau: A Note on Niskanens Theory of Bureaucracy, in: Journal of Political Economy 83, $S$. 195-207

BROWNING, E. (1974): Why the Social Insurance Budget is to Large in a Democracy, in: Economic Inquiry 13, S. $373-388$

BUCHANAN, J. (1962): Politics, Policy and Pigovian Margins, in: Economica 29, S. 17-28

BUCHANAN, J. (1967): Public Finance in Democratic Process, Chapel Hills

BUCHANAN, J. (1975a): A Contractarian Paradigma for Applying Economic Theory, in: American Economic Review, Papers and Proceedings 65 , S. 225-230

BUCHANAN, J. (1975b): The Limits of Liberty. Between Anarchy and Leviathan, Chapel Hills

BUCHANAN, J. (1980): Procedural and Quantitative Constitutional Constraints on Fiscal Authority, in: MOORE, W. /PENNER, R. (eds.): The Constitution and the Budget, Washington D.C./London, S. $80-84$

BUCHANAN, J./FLOWERS, M. (1969:) An Analytical Setting für a 'Taxpayer's Revolution', in: Western Economic Journal $7, S$. $349-359$ 
BUCHANAN, J./TOLLISON, G./TULLOCK, G. (1980): Toward a Theory of Rent Seeking Society, College Station

BUCHANAN, J./TULLOCK, G. (1962): The Calculus of Consent, Ann Arbor

BUCHANAN, J./WAGNER, R. (1977): Democracy in Deficit. The Political Legacy of Lord Keynes, New York/San Francisco/London

BUCHANAN, J./WAGNER, R. (1978a): The Political Biases of Keynesian Economics, in: BUCHANAN, J./WAGNER, R. (eds.): Fiscal Responsibility in Constitutional Democracy, Boston, S. 79-100

BUCHANAN, J./WAGNER, R. (1978b): Democracy and Keynesian Constitutions: Political Biases and Economic Consequences, in: BUCHANAN, J./BURTON, J. /WAGNER, R.: The Consequences of Mr. Keynes, London, S. $11-27$

BUSH, W./DENZAU, A. (1977): The Voting Behaviour of Bureaucrats and Public Sector Growth, in: BORCHERDING, Th. (ed.):Budgets and Bureaucrats: The Sources of Government Growth, Durham, S. 90-99

CARTER, R. (1982): Beliefs and Errors in Voting Choices: A Restatement of the Theory of Fiscal Illusion, in: Public Choice $39, \mathrm{~S}$. $343-360$

COURANT, P./GRAMLICH, E./RUBINFELD, L. (1979): TaX Limitation and the Demand for Public Services in Michigan, in: National Tax Journal 32, Supp 1., S. 147-157

COURANT, P./GRAMLICH, E./RUBINFELD, L. (1981): Why Voters Support Tax Limitation Amendments: the Michigan Case, in: LADD, H./TIDEMAN, T. (eds.): Tax and Expenditure Limitations, Washington D.C., S. $37-72$

DARBY, M./KARNI, E. (1973): Free Competition and the Optimal Amount of Fraud, in: Journal of Law and Economics 16, S. 67-88

DAVIS, 0./WHINSTON, A. (1962): Externalities, Welfare and the Theory of Games, in: Journal of Political Economy 70 , S. 241-262 
DAVIS, 0./WHINSTON, A. (1967): On the Distinction Between Private and Public Goods, American Economic Review 57 , S. $360-373$

DENZAU, A./MACKAY, R. (1976): Benefit Shares and Majority Voting, in: American Economic Review 66 , S. $69-76$

DENZAU, A./MACKAY, R. (1980a): A Model of Benefit and Tax Share Discrimination, in: Journal of Public Economics 13, S. 341-368

DENZAU, A./MACKAY, R. (1980b): Limits on Public Provision of Private Goods: Reply and Further Analysis, in: American Economic Review 70 , S. $466-473$

DENZAU, A./MACKAY, R./WEAVER, C. (1979): Spending Limitations, Agenda Control and Voter's Expectations, in: National Tax Journal 32, Suppl., S. 189-200

DENZAU, A./MACKAY, R./WEAVER, C. (1981): On the Initiative Referendum Option and the Control of Monopoly Government, in: LADD, H./TIDEMAN, T. (eds.): Tax and Expenditure Limitations, Washington D.C., S. $191-222$

DOWNS, A. (1960): Why the Government Budget is to Small in a Democracy, in: World Politics 13 , S. 541-563

DowNS, A. (1968): Okonomische Theorie der Politik, Tübingen

DWORAK, R. (1980): Taxpayers, Taxes and Government Spending - Perspectives on the Tax Revolt, New York

FILIMON, R./ROMER, R./ROSENTHAL, H. (1982): Asymetric Information and Agenda Control: the Bases of Monopoly Power in Public Spending, in: Journal of Public Economics 17 , S. 51-70

FLOWERS, M. (1977): Revenue Structure, Fiscal Illusion and Budgetary Choice: Comment, in: Public Choice 29, S. 127-131

FOLKERS, C. (1983a): Begrenzung von Steuern und Staats ausgaben in den USA, Baden-Baden 
FOLKERS, C (1983b): Zu einer positiven Theorie der Steuerreform, in: HANSMEYER, H.K. (Hrsg.): Statsfinanzierung im Wandel, Schriften des Vereins für Socialpolitik N.F. Bd. 134, Köln, S. $189-211$

FREIMAN, M./GRASSO, P. (1982): Budget Impact and Voter Response to Tax Limitation Referenda, in: Public Finance Quarterly 10 , S. $49-66$

FREY, B. (1970): Models of Perfect Competition and Pure Democracy, in: Kyklos 23 , S. $736-755$

FREY, B./POMMEREHNE, W. (1982): How Powerful Are Public Bureaucrats?, in: Public Choice 38 , S. 253-262

GALBRAITH, K. (1958): The Affluent Society, Boston GOETZ, Ch. (1977): Fiscal Illusion in State and Local Finance, in: BORCHERDING, Th. (ed.): Budgets and Bureaucrats: The Sources of Government Growth, Durham, S. $176-185$

GROSSEKETTLER, H. (1981): Der Brennan-Buchanan-Plan. zur Eindämmung der Statstätigkeit oder: Brauchen wir eine neue Finanzwissenschaft?, in: Finanzarchiv, N.F. 39 , S. $495-508$

GUNTHER, G. (1980): Constitutional Roulette: The Dimension of Risk, in: MOORE, W./PENNER, R. (eds.): The Constitution and the Budget, Washington D.C., S. 5-9

HAYEK, F. (1945): The Use of Knowledge in Society, in: American Economic Review 35 , S. $519-530$

HERDER-DORNEICH, Ph. (1959): Politisches Modell zur Wirtschaftstheorie, Freiburg

HERDER-DORNEICH, Ph./GROSER, M. (1977): Jkonomische Theorie des politischen Wettbewerbs, Göttingen

HUNTER, W. (1983): Tax Structure and Bureaucratic Bargaining, in: Public Finance Quarterly 11, S. 347-364 
JACKSON, R. (1971): A 'Taxpayer's Revolution' and Economic Rationality, in: Public Choice 10, S. 93-96

KIRLIN, J. (1982): The Political Economy of Fiscal Limits, Lexington

KNAPPE, E. (1980): Einkommensumverteilung in der Demokratie, Freiburg

KRAMER, G. (1973): On a Class of Equilibrium Conditions for Majority Rule, in: Econometrica 41 , S. 285-297

KRAMER, G. (1977): A Dynamical Model of Political Equilibrium, in: Journal of Economy Theory 16 , S. 310-334

KRAUSE-JUNK, G. (1972): Probleme der Berechnung und Schätzung öffentlicher Ausgaben, in: Public Finance 28, S. 127-144

LEIBENSTEIN, H. (1966): Allocative Efficiency vs. "X-Efficiency", in: American Economic Review 56 , S. $392-415$

LIEFMANN-KEIL, E. (1969): Zur Aktivität der Interessenverbände, in: ARNDT, H. (Hrsg.): Sozialwissenschaftliche Untersuchungen, Berlin, S. 27-61

LINDSAY, C./NORMAN, D. (1977): Reopening the Question of Government Spending, in: BORCHERDING, Th. (ed.): Budgets and Bureaucrats: The Sources of Government Growth, Durham, S. 260-274

LIPSEY, R./LANCASTER, K. (1956): The General Theory of Second Best, in: Review of Economic Studies 24 , S. 11-32

LITTLE, I. (1950): A Critique of Welfare Economics, oxford

MACKAY, R./WEAVER, C. (1978): Monopoly Bureaus and Fiscal outcomes: Deductive Models and Implications for Reform, in: TULLOCK, G. /WAGNER, R. (eds.): Policy Analys is and Deductive Reasoning, Lexington, S. $141-165$

MACKAY, R./WEAVER, C. (1981): Agenda Control by Budget Maximizers in a Multi-Bureau Setting, in: Public Choice 37, 1981, S. $447-472$ 
MCCORMICK, R./TOLLISON, R. (1980): Wealth Transfers in a Representative Democracy, in: BUCHANAN/TOLLISON/TULLOCK (1980), S. $293-313$

MCCORMICK, R./TOLLISON, R. (1981): Politicians, Legislation and the Economy: An Inquiry into the Interest-Group Theory of Government, Boston

MCGUIRE, Th./COINER, M./SPANCAKE, L. (1979): Budget-Maximizing Agencies and Efficiency in Government, in: Public Choice $34, \mathrm{~S}$. $333-357$

MCKELVEY, R. (1976.): Intransitivities in Multidimensional Voting Models and Some Implications for Agenda Control, in: Journal of Economic Theory 12 , S. 472-482

MELTZER, A./RICHARD, S. (1981): A Rational Theory of the Size of Government, in: Journal of Political Economy 89, S. 914-927

MIGUE, J./BELANGER,G. (1974): Toward a General Theory of Managerial Discretion, in: Public Choice 17 , S. $27-43$

MUELLER, D. (1977): Allocation, Distribution and Collective Choice, in: Public Finance 32 , S. $225-244$

MUELLER, D. (1979): Public Choice, Cambridge

MURPHY, D./RUBART, F./MOLLER, F./RASCHKE, J. (1979): Protest: Grüne, Bunte und die Steuerrebellen - Ursachen und Perspektiven -, Reinbek

MUSGRAVE, R. (1969): Finanztheorie, Tübingen

MUSGRAVE, R. (1981): Leviathan Cometh-or does he?, in: LADD, H./TIDEMAN, T. (eds.): Tax and Expenditure Limitations, Washington D.C., S. $77-120$

NELSON, D. (1976): Political Information, in: Journal of Law and Economics 19, S. 315-336

NISKANEN, W. (1968): Non Market Decisions - The Peculiar Economics of Bureaucracy, in: American Economic Review, Papers and Proceedings 58, S. 293-305 
NISKANEN, W. (1971): Bureaucracy and Representative Government, Chicago

NISKANEN, W. (1975): Bureaucrats and Politicians, in: Journal of Law and Economics $18, \mathrm{~S}$. $617-643$

OEHSEN, J.H. von (1982): Optimale Besteuerung - Optimal Taxation -, Frankfurt/Bern/Las Vegas

OLSON, M. (1965): The Logic of Collective Action, Cambridge

ORZECHOWSKI, W. (1977): Economic Models of Bureaucracy: Survey, Extensions and Evidence, in: BORCHERDING, Th. (ed.): Budgets and Bureaucrats: The Sources of Government Growth, Durham, S. 229-359

PEACOCK, A. (1980): On the Anatomy of Collective Failure, in: Public Finance 35 , S. 33-43

PELTZMAN, S. (1976): Towards a More General Theory of Regulation, in: Journal of Law and Economics 19, S.211-240

POMMEREHNE, W./SCHNEIDER, F. (1978): Fiscal Illusion, Political Institutions and Local Public Spending, in: Kyklos $31, S$. $381-408$

POSNER, R. (1971): Taxation by Regulation, in: Bell Journal of Economics and Management Science 2, S. 22-50

POSNER, R. (1974): Theories of Economic Regulation, in: Bell Journal. Of Economics and Manegement Science 5, S. 335-358

POSNER, R. (1975): The Social Costs of Monopoly and Regulation, in: Journal of Political Economy 83, S.807-827

PUVIANI, A. (1960): Die Illusionen in der öffentlichen Finanzwirtschaft. Berlin

RAE, D.(1969): Decision-Rules and Individual Values in Constitutional Choice, in: American Political Science Review 63, S. $40-56$

RAPAPORT, A./CHAMMAH, A. (1965): Prisoner's Dilemma. A Study in Conflict and Cooperation, Ann Arbor 
RAWLS, J. (1971): A Theory of Justice, Cambridge

RECKTENWALD, H. (1980): Zur Theorie des Staatsversagens, in: Public Finance $35,5.72-78$

REID, J.(1979): Tax Revolts in Historical Perspective,in: National Tax Journal 32 , Suppi., S. $67-74$

RIKER, W. (1980): Implications from the Disequilibrium of Majority Rule for the Studies of Institutions, in: American Political Science Review 74, S. 432-446

RIKER,W./ORDESHOOK, P. (1968): A Theory of the Calculus of Voting, in: American Political Science Review 62, S. 25-42

ROMER,T./ROSENTHAL, H. (1978): Political Ressource Allocation, Controlled Agenda and The Status Quo, in: Public Choice $33, \mathrm{~S}$. $27-43$

ROMER, T./ROSENTHAL, H. (1979): Bureaucrats Versus Voters: On the Political Economy of Ressource Allocation by Direct Democracy, in: Quarterly Journal of Economics 93, S. $563-587$

ROPPEL, U. (1979): бkonomische Theorie der Bürokratie. Freiburg

ROSE-ACKERMAN, S. (1975): The Economics of Corruption, in: Journal of Public Economics 4,5 . $187-203$

ROSE-ACKERMAN, S. (1978): Corruption: A Study in Political Economy. San Francisco/London/New York

ROSE-ACKERMAN, S. (1980): Inefficiency and Reelection, in: Kyk ios 33, S. 287-307

ROSENSCHON, A. (1980): Verschwendung im staat und am Markt. Göttingen

RUBIN, P. (1975): On the Form of Special Interest Legislation, in: Public Choice 21, S. $79-90$

SAMUELSON, P. (1954): The Pure Theory of Public Expenditure, in: Review of Economics and Statistics 36 , S. $387-389$ 
SCHMIDT, K.(1966): Entwicklungstendenzen der öffentlichen Ausgaben im demokratischen Gruppenstaat, in: Finanzarchiv N.F. 25 , S. $213-241$

SCHMIDT, K. (1969): Zur okonomik der Korruption, in: Schmollers Jahrbuch für Wirtschafts und Sozialwissenschaften 89 , S.129-149

SCHMIDT, K.(1978): Artike l: Korruption, in: Handwörterbuch der Wirtschaftswissenschaften Bd. 6, S. 565-573

SCHMOLDERS, G. (1959): Unmerkliche Steuern, in: Finanzarchiv N.F. $20,5.23-34$

SCITOVSKY, T. (1941/42): A Note on Welfare Propositions in Economics and a Reconsideration of the Theory of Tariffs, in: Review of Economic Studies 9, S.77-110

SENF, P. (1976): Kurzfristige Haushaltsplanung, in: Handbuch der Finanzwissenschaft, 3 . Auf 1., Bd. 1, S. 371-425

SHAPIRO, P. (1981): Popular Response to Public Spending Disequilibrium: An Analys is of the 1978 California Property Tax Limitation Initiative, in: LADD, H./TIDEMAN, T. (eds.): Tax and Expenditure Limitations, Washington D.C., S. $13-35$

SHAPIRO, P./PURYEAR, D./ROSS, J. (1979): Tax and Expenditure Limitations in Retrospect and Prospect, in: National Tax Journal 32 , Supp I., S.1-10

SHUBIK, M. (1968): A Two Party System,General Equilibrium and the Voter's Paradox, in: Zeitschrift für Nationalökonomie 28 , S. $341-354$

SOHMEN, E.(1976): Allokationstheorie und Wirtschaftspolitik, Tübingen

STAAF, R./WEST, E. (1980): Limits on Public Provision of Private Goods, in: American Economic Review 70, S.461-465

STERN, K. (1982/83): Verfassungsrechtliche Aspekte der Statsverschuldung unter Berücksichtigung von Art. $115 \mathrm{GG}$, in: Steuerberater-Jahrbuch $1982 / 83$, S. 42-68 
STIGLER, G. (1971): The Theory of Economic Regulation, in: Bell Journal of Economics and Management Science 2, S.3-21

TAYLOR, M.(1969): Proof of a Theorem on Majority Rule, in: Behavioral Science 14, S.228-231

TAYLOR, M. (1976): Anarchy and Cooperation, London

TOLLISON, R. (1982): Rent Seeking: A Survey, in: Kyklos 35 , S. 575-602

TOLLISON, R./CRAIN, M./PAUTLER, P. (1975): Information and Voting: An Empirical Note, in: Public Choice $24,5.43-49$

TOLLISON, R./WILLETT, T. (1973): Some Simple Economics of Voting and Not Voting, in: Public Choice 16, S.59-71

TOMA, M./TOMA, E. (1980): Bureaucratic Responses to Tax Limitation Amendments, in: Public Choice 35 , S. $333-348$

TUERCK, D. (1978): The Theory of Political Advertising, in: TUERCK, D. (ed.): The Political Economy of Advertising. Washington D.C., S.61-80

TULLOCK, G. (1959): Some Problems of Majority Voting, in: Journal of Political Economy 67 , S. $571-579$

TULLOCK, G. (1967): Towards a Mathematics of Politics, Ann Arbor

TULLOCK, G. (1970): A Simple Algebraic Logrolling Model, in: American Economic Review 60 , S. $419-426$

TULLOCK, G. (1971): The Costs of Transfers, in: Kyklos $24,5.629-643$

TULLOCK, G. (1972): Review of Niskanen's 'Bureaucracy and Representative Government', in: Public Choice 12, S. 119-124

TULLOCK, G. (1974): Dynamic Hypothes is of Bureaucracy, in: Public Choice 19, S. 127-131

TULLOCK, G. (1975): The Transitional Gains Trap, in: Bell Journal of Economics 6, S. 671-678 
TULLOCK, G. (1977): What Is To Be Done, in: BORCHERDING, Th. (ed.): Budgets and Bureaucrats: The Sources of Government Growth, Durham, S.275-288

TULLOCK, G. (1980): Rent Seeking as a Negative Sum Game, in: BUCHANAN/TOLLISON/TULLOCK (1980), S. $16-36$

WAGNER, R. (1966): Pressure Groups and Political Enterpreneurs: A Review Article, in: Papers on Non-Market Decision Making 1. S. $161-170$

WAGNER, R. (1976): Revenue Structure, Fiscal Illusion and Budgetary Choice, in: Public Choice 25, S.45-61

WAGNER, R. (1978): Advertising and the Public Economy: Some Preliminary Ruminations, in: TUERCK, D. (ed.): The Political Economy of Advertising, Washington D.C., S. 81-100

WAGNER, R./WEBER,W. (1975): Competition, Monopoly and the Organization of Government in Metropolitan Areas, in: Journal of Law and Economics 18, S.661-684

WEINGAST, B./SHEPSLE, K./JOHNSON, Ch. (1981): The Political Economy of Benefits and Costs: A Neoclassical Approach to Distributive Politics, in: Journal of Political Economy 89 , S.642-663

WEST, E./WINER, St. (1980): Optimal Fiscal Illusion and the Size of Government, Public Choice 35, S.607-622

WILDAVSKY, A. (1980): How To Limit Government Spending, Berkeley/Los Angeles/London

WILLIAMSON, 0. (1964): The Economics of Discretionary Behavior: Managerial Objectives in a Theory of Firm, Englewood Cliffs

WOLF,Ch. (1979): A Theory of Nonmarket Failure: Framework for Implementation Analysis, in: Journal of $L a w$ and Economics 22 , S.107-137

WYSE, A./DAVIES, D. (1978): A Bayesian Approach to Advertising as Information, in: TUERCK, D. (ed.): The Political Economy of Advertising, Washington D.C., S. 107-132 


\section{FINANZWISSENSCHAFTLICHE SCHRIFTEN}

Band 1 Werner Steden: Finanzpolitik und Einkommensverteilung. Ein Wachstums- und Konjunkturmodell der Bundesrepublik Deutschland. 1979.

Band 2 Rainer Hagemann: Kommunale Finanzplanung im föderativen Staat. 1976.

Band 3 Klaus Scherer: Maßstäbe zur Beurteilung von konjunkturellen Wirkungen des öffentlichen Haushalts. 1977.

Band 4 Brita Steinbach: „Formula Flexibility” - Kritische Analyse und Vergleich mit diskretionärer Konjunkturpolitik. 1977.

Band 5 Hans-Georg Petersen: Personelle Einkommensbesteuerung und Inflation. Eine theoretisch-empirische Analyse der Lohn- und veranlagten Einkommensteuer in der Bundesrepublik Deutschland. 1977.

Band 6 Friedemann Tetsch: Raumwirkungen des Finanzsystems der Bundesrepublik Deutschland. Eine Untersuchung der Auswirkungen der Finanzreform von 1969 auf die Einnahmenposition der untergeordneten Gebietskörperschaften und ihrer regionalpolitischen Zieladäquanz. 1978.

Band 7 Wilhelm Pfähler: Normative Theorie der fiskalischen Besteuerung. Ein methodologischer und theoretischer Beitrag zur Integration der normativen Besteuerungstheorie in der Wohlfahrtstheorie. 1978.

Band 8 Wolfgang Wiegard: Optimale Schattenpreise und Produktionsprogramme für öffentliche Unternehmen. Second-Best-Modelle im finanzwirtschaftlichen Staatsbereich. 1978.

Band 9 Hans P. Fischer: Die Finanzierung des Umweltschutzes im Rahmen einer rationalen Umweltpolitik. 1978.

Band 10 Rainer Paulenz: Der Einsatz finanzpolitischer Instrumente in der Forschungs- und Entwicklungspolitik. 1978.

Band 11 Hans-Joachim Hauser: Verteilungswirkungen der Staatsverschuldung. Eine kreislauftheoretische Inzidenzbetrachtung. 1979.

Band 12 Gunnar Schwarting: Kommunale Investitionen. Theoretische und empirische Untersuchungen der Bestimmungsgründe kommunaler Investitionstätigkeit in Nordrhein-Westfalen 1965-1972. 1979.

Band 13 Hans-Joachim Conrad: Stadt-Umland-Wanderung und Finanzwirtschaft der Kernstädte. Amerikanische Erfahrungen, grundsätzliche Zusammenhänge und eine Fallstudie für das Ballungsgebiet Frankfurt am Main. 1980.

Band 14 Cay Folkers: Vermögensverteilung und staatliche Aktivität. Zur Theorie distributiver Prozesse im Interventionsstaat. 1981.

Band 15 Helmut Fischer: US-amerikanische Exportförderung durch die DISC-Gesetzgebung. 1981.

Band 16 Günter Ott: Einkommensumverteilungen in der gesetzlichen Krankenversicherung. Eine quantitative Analyse. 1981.

Band 17 Johann Hermann von Oehsen: Optimale Besteuerung. (Optimal Taxation). 1982.

Band 18 Richard Kössler: Sozialversicherungsprinzip und Staatszuschüsse in der gesetzlichen Rentenversicherung. 1982.

Band 19 Hinrich Steffen: Zum Handlungs- und Entscheidungsspielraum der kommunalen Investitionspolitik in der Bundesrepublik Deutschland. 1983.

Band 20 Manfred Scheuer: Wirkungen einer Auslandsverschuldung des Staates bei flexiblen Wechselkursen. 1983.

Band 21 Christian Schiller: Staatsausgaben und crowding-out-Effekte. Zur Effizienz einer Finanzpolitik keynesianischer Provenienz. 1983. 
Band 22 Hannelore Weck: Schattenwirtschaft: Eine Möglichkeit zur Einschränkung der öffentlichen Verwaltung? Eine ökonomische Analyse. 1983.

Band 23 Wolfgang Schmitt: Steuern als Mittel der Einkommenspolitik. Eine Ergänzung der Stabilitätspolitik? 1984.

Band 24 Wolfgang Laux: Erhöhung staatswirtschaftlicher Effizienz durch budgetäre Selbstbeschränkung? Zur Idee einer verfassungmäßig verankerten Ausgabengrenze. 1984. 
Gerhold, Karl

DIE BEDEUTUNG DES ANGEBOTSWACHSTUMS FƯR DIE GELDWERTENTWICKLUNG

Eine Stagflationserklärung für die Bundesrepublik Deutschland

Frankfurt/M., Bern, 1982. IV, 245 S.

Europäische Hochschulschriften: Reihe 5, Volks- und Betriebswirtschaft. Bd. 397

ISBN 3-8204-7300-9

br. sFr. 57.-

In der vorliegenden Arbeit wird zur Erhellung des Stagflationsphänomens im Gegensatz zum üblichen Vorgehen nicht auf das Wirksamwerden exogener Faktoren, sondern auf den Zusammenhang zwischen Mengen und Preisen selbst abgestellt.

Mit den Mitteln der empirischen und der theoretischen Analyse zeigt der Verfasser, dass für die Bundesrepublik Deutschland folgende These Erklärungswert besitzt: Eine sich relativ stetig entwickelnde Nachfrage steht stärker schwankenden und im Trend abnehmenden realen Wachstumsraten gegenüber. Die durch die Nachfrageentwicklung festgelegte Preissteigerungstendenz führt in dem Masse zu einem Preisniveauanstieg in dem reales Wachstum nicht stattfindet.

Aus dem Inhalt: Okonometrische und theoretische Untersuchung der Wirkungen des Angebotswachstums auf die Geldwertentwicklung - Untersuchung der empirischen und der theoretischen Relevanz anderer Geldwerterklärungen.

Schiller, Christian

\section{STAATSAUSGABEN UND CROWDING-OUT-EFFEKTE}

Zur Effizienz einer Finanzpolitik keynesianischer Provenienz

Frankfurt/M., Bern, 1983. XI, 204 S.

Finanzwissenschaftliche Schriften. Bd. 21

ISBN 3-8204-7503-6

br. sFr. 49.-

Unter dem Eindruck der Erfahrungen des letzten Jahrzehnts und vorher schon aufgrund theoretischer Uberlegungen sind mehr und mehr Zweifel aufgekommen, ob eine finanzwirtschaftliche Nachfragesteuerung ein taugliches Mittel gegen Unterbeschäftigung ist. Ziel der vorliegenden Arbeit ist es, die theoretischen Erwägungen aufzuarbeiten und im Hinblick auf ihre Voraussetzungen und deren Plausibilität zu überprüfen.

Aus dem Inhalt: Dogmenhistorische Wurzeln der crowding-out-These - Formen des crowdingout - Zu den Ursachen des crowding-out - Der Hickssche crowding-out-Effekt - Vermögenseffekte - Ausgabeneffekte - Preiseffekte - Erwartungseffekte.

\section{Verlag Peter Lang Bern $\cdot$ Frankfurt a.M. $\cdot$ New York \\ Auslieferung: Verlag Peter Lang AG, Jupiterstr. $15, \mathrm{CH}-3000$ Bern 15 Telefon (0041/3I) 321122. Telex verlch 32420}

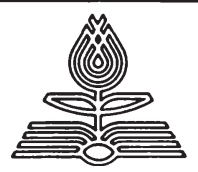


Folkers, Cay

\section{VERMOGENSVERTEILUNG UND STAATLICHE AKTIVITÄT}

Zur Theorie distributiver Prozesse im Interventionsstaat

Frankfurt/M., Bern, 1981. VIII, 380 S.

Finanzwissenschaftliche Schriften. Bd. 14

ISBN 3-8204-6191-4

br. sFr. 49.-

Die Untersuchung hat das Ziel, eine theoretische Analyse der Funktionen und Bestimmungsgründe der Vermögensverteilung unter besonderer Berücksichtigung der Beziehungen zu den ökonomischen Aktivitäten des Staates zu entwerfen. Sie will zu einer Neuformulierung von Konzepten und Systemzusammenhängen der Vermögensverteilung beitragen, um eine fundierte Beurteilung verteilungspolitischer Massnahmen des Staates bezüglich des Vermögens zu ermöglichen.

Aus dem Inhalt: U.a. Die Vermögensfunktionen - Die langfristige Inzidenz vermögenspolitischer Massnahmen - Die private Vermögensverteilung aus neoklassischer und neokeynesianischer Sicht - Machtfunktion und Vermögensverteilung - Die Vermögensverteilung zwischen ökonomischen Abhängigkeiten und staatlichem Eingriff - Private Vermögensverteilung und öffentliches Vermögen.

Scholz, Eberhard

STAATLICHE SPARFÓRDERUNG, VERMOGGENSBESTEUERUNG UND PERSONELLE VERMOGGENSVERTEILUNG IN MAKROOKONOMISCHER WIRKUNGSANALYSE

Frankfurt/M., Bern, 1981. $161 \mathrm{~S}$.

Europäische Hochschulschriften: Reihe 5, Volks- und Betriebswirtschaft. Bd. 347

ISBN 3-8204-5998-7

br. sFr. 34.-

Die vorliegende Arbeit behandelt am Beispiel vermögensteuerfinanzierter Sparprämien die Wirkungen staatlicher vermögenspolitischer Massnahmen auf die personelle Vermögensverteilung. Zunächst wird ein makroökonomisches 3-Gruppen-Grundmodell der Vermögensbildung durch Sparen analysiert, das im Laufe der Untersuchung durch Berücksichtigung der Vermögensstruktur und von Vermögensübertragungen erweitert wird. Mit der Analyse der dynamischen Stabilität der Modelle treten die Bedingungen, unter denen zielkonforme Wirkungen der untersuchten Instrumente auftreten, deutlich hervor.

Aus dem Inhalt: Ein dynamisches Grundmodell der Vermögensbildung und Vermögensverteilung - Erweiterung des Grundmodells durch Berücksichtigung der Vermögensstruktur - Berücksichtigung von Vermögensübertragungen.

\section{Verlag Peter Lang Bern - Frankfurt a.M. New York \\ Auslieferung: Verlag Peter Lang AG, Jupiterstr. 15, CH-3000 Bern 15 Telefon $(0041 / 31) 32$ II 22, Telex verlch 32420}

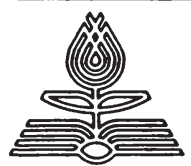


Wolfgang Laux - 978-3-631-75220-3

Downloaded from PubFactory at 01/11/2019 06:56:13AM

via free access 
Wolfgang Laux - 978-3-631-75220-3

Downloaded from PubFactory at 01/11/2019 06:56:13AM

via free access 